\title{
un modèle de sols saturés en dynamique non linéaire
}

\section{a model for the non linear dynamic analysis of saturated soils}

\author{
D. AUBRY \\ Ecole Centrale de Paris \\ H. MODARESSI \\ Bureau de Recherches Géologiques et Minières *o
}

Rev. Franç. Géotech. n 46 , p.p. $43-75$ (janvier 1989)

\section{Résumé}

Un modèle pour étudier l'influence des facteurs géologiques sur les réponses sismiques des sites constitués des sols déformables est présenté.

La première partie est consacrée à la formulation physique des milieux biphasiques saturés et à la modélisation du comportement élastoplastique des sols. Le comportement biphasique des sols est modélisé par une théorie de Biot affinée à l'aide des méthodes d'homogénéisation. Une formulation simplifiée permettant une analyse moins coûteuse et valable pour les phénomènes à basse fréquence est mise en œuvre. Le comportement élastoplastique des sols est décrit par une loi élastoplastique multimécanisme cyclique.

Dans la modélisation en éléments finis des problèmes de propagation des ondes, une attention particulière est portée sur les limites du maillage. Le développement d'éléments paraxiaux permet de construire de telles frontières absorbantes. Ils constituent également un moyen intéressant pour introduire le champ incident sismique généralisé.

La présentation est achevée par quelques exemples mono et bidimensionnels pour montrer l'intérêt de cette approche pour l'évaluation directe de la réponse sismique des sites.

\section{Abstract}

A methodology regarding the seismic response of sites and the influence of geological factors is presented.

The method combines a Biot's theory and an elastoplastic constitutive model for soils and a unified approach of the seismic incident field and absorbing boundaries.

A brief review of homogeneization methods applied to saturated porous media is given. The obtained formulae are compared with those derived from Biot's theory and the important aspects of an elastoplastic multimechanism cyclic model are outlined.

Finally, this methodology is applied to some one and two dimensional examples including site effects and the results are discussed. 
Bien que l'importance de l'eau en tant que fluide interstitiel soit toujours reconnue dans les présentations classiques de mécanique des sols, hormis le cas de la consolidation de Terzaghi et peut-être celui de l'estimation des pressions interstitielles grâce aux coefficients de Skempton, le comportement couplé des phases fluide et solide est très rarement modélisé, en particulier dans le cas de chargements dynamiques. Cependant, les progrès réalisés dans la description du comportement cyclique des sols en contraintes effectives et des variations de volume, alliés à des techniques numériques modernes donnent tous les éléments pour construire un modèle biphasique reproduisant l'essentiel des phénomènes observés : tassements irréversibles, liquéfaction pendant la sollicitation dynamique ou post-sismique.

Dans ce travail, les bases d'un tel modèle prenant réellement en compte le comportement du milieu poreux et du fluide interstitiel sont présentées. Des applications à l'estimation des effets sismiques de site sont discutées. Les détails des modèles, de la mise en cuuve numérique et des exemples sont donnés dans la thèse de $\mathrm{H}$. MODARESSI (1987). Plus précisément plusieurs aspects d'une modélisation assez complète sont abordés : rôle couplé du fluide interstitiel et des déformations du milieu poreux en dynamique, comportement non élastique des sédiments, champ libre compatible avec les conditions locales de site, modélisation du domaine infini par rapport aux hétérogénéités locales.

\section{Rôle couplé du fluide interstitiel et des déformations du milieu poreux en dynamique}

La première formulation permettant d'étudier l'écoulement d'un fluide à travers un milieu poreux déformable et saturé a été présentée par TERZAGHI (1930) dans sa théorie de la consolidation en introduisant la notation de contrainte effective. BIOT (1941) a généralisé cette théorie, en prenant en compte la compressibilité du fluide supposé parfait et de la matrice solide ayant un comportement élastique linéaire. Il a étendu ensuite cette formulation aux problèmes dynamiques et en particulier la propagation des ondes dans les milieux poreux saturés. L'existence d'une onde de compression supplémentaire dite * lente a a été mise en évidence par cette théorie (BIOT, 1956). A basses fréquences les termes de couplage visqueux, (autrement dit le tenseur de perméabilité dans la loi de Darcy) sont indépendants de la fréquence et prédominants par rapport aux forces inertielles. Le passage d'un comportement à l'autre est défini par une certaine fréquence caractéristique, fonction de la viscosité cinématique du fluide et de la géométrie des pores. La théorie des mélanges (BOWEN, 1976) est en bonne concordance avec la théorie de Biot en petite déformation pour un solide élastique et un fluide parfait. ZIENKIEWICZ et al. (1984) ont présenté une formulation simplifiée des équations de Biot en dynamique (formulation $u_{s}-p$ ), applicable aux problèmes en basses fréquences, où on néglige l'accélération de la phase fluide par rapport à l'accélération du squelette solide. $\mathrm{Ce}$ dernier modèle est très intéressant, car il permet de réduire le nombre de degrés de liberté dans un calcul numérique. Ces mêmes auteurs établissent pour un pro- blème monodimensionnel, les limites d'application des différentes formulations.

L'eau joue trois rôles très importants sur les réponses sismiques dans un site constitué de sols déformables. Premièrement par l'intermédiaire de la pression interstitielle, elle modifie le comportement des sols en jouant sur l'état des contraintes effectives; deuxièmement elle modifie le champ d'onde en particulier lorsque les ondes dites * lentes * sont créées et enfin, la nature dissipative du phénomène de filtration augmente l'amortissement global du système. L'interface entre les couches considérées biphasiques et les couches monophasiques a une influence importante sur les résultats. Pour une étude plus détaillée des conditions sur linterface, nous renvoyons à GELI (1985).

\section{Comportement non élastique des sédiments}

La prise en compte des effets de non linéarité du comportement des sols sur la réponse sismique des sites dans les modélisations numériques date des années 1960. IDRISS et SEED (1968) ont proposé la méthode viscoélastique linéaire équivalente. Cette méthode très répandue depuis, dans les modélisations numériques, a été très souvent l'objet d'études de comparaison et largement détaillée dans la littérature (PECKER, 1985). Cette méthode est basée sur l'hypothèse que la réponse non linéaire du sol peut être approchée par un modèle linéaire ayant les mêmes propriétés dissipatives. Cette méthode s'avère insuffisante pour les mouvements sismiques forts et est mal adaptée lorsque des déformations irréversibles importantes sont produites dans les sols. En général pour les sollicitations élevées, la méthode équivalente linéaire sous-estime l'intensité des mouvements à la surface de sol pour les périodes allant de 0.1 à 0.5 secondes.

Une approche réellement non linéaire a été présentée par JOYNER et CHEN (1975), en utilisant un modèle de comportement composé d'une série de ressorts et de patins plastiques. Dans cette approche la dissipation d'énergie est due au comportement hystérétique du sol et il n'y a pas d'amortissement visqueux. Ils observent globalement que leur modèle reproduit correctement le champ des vitesses, qu'il sous-estime la valeur de l'accélération maximale produite pour les hautes fréquences et qu'il amplifie les accélérations dans le domaine des basses fréquences.

\section{Champ libre compatible avec les hétérogénéités locales du site}

L'influence des irrégularités géométriques et des hétérogénéités latérales ne peut pas être mise en évidence par les calculs monodimensionnels. Depuis longtemps il a été reconnu que les irrégularités souterraines, ainsi que le 
comportement anélastique des sols modifient les signaux sismiques. Les résultats théoriques présentés pour les vallées alluviales semi-circulaires montrent que la géométrie de ces irrégularités peut sensiblement modifier la réponse à la surface et un calcul monodimensionnel s'avère insuffisant pour modéliser le champ libre local. Les amplifications obtenues par les calculs bidimensionnels peuvent être plus grandes que celles obtenues par les calculs monodimensionnels.

Dans les problèmes sismiques, la fréquence est de lordre de $0.1 \mathrm{~Hz}$ à $30 \mathrm{~Hz}$ et la vitesse des ondes à proximité de la surface du sol est de $0.1 \mathrm{~km} / \mathrm{s}$ à $3 \mathrm{~km} / \mathrm{s}$. Par conséquent, les longueurs d'onde varient de quelques dizaines de mètres à quelques dizaines de kilomètres. Les irrégularités géologiques ou topographiques, ayant des dimensions de cet ordre, ont des influences considérables sur la réponse sismique du sol. Les méthodes analytiques présentées dans la littérature pour résoudre des problèmes d'amplification dus aux irrégularités topographiques ne considèrent souvent que le cas des ondes SH. Lorsqu'on est en présence des ondes P et SV le problème est plus compliqué, car ces ondes restent couplées par les conditions de réflexion-transmission. BARD et BOUCHON (1980) et BARD (1982 et 1983) ont étendu la méthode de Aki et Larner dans le domaine transitoire, et ont mis en évidence l'importance des ondes diffractées par des irrégularités topographiques, donnant naissance à des ondes de surface locales, même lorsque l'onde incidente se propage verticalement. Par ailleurs, la méthode de différences finies a été utilisée par BOORE (1971) et en particulier par BOORE, HARMSEN et HARDING (1981) pour étudier la diffraction des ondes sur une falaise. La méthode des équations intégrales a été très souvent utilisée pour étudier linfluence des irrégularités géométriques (WONG, 1982 ; SANCHEZ-SEZMA, 1982 et DRAVINSKI (1982 et 1983). La méthode des éléments finis au contraire a été rarement utilisée pour déterminer l'influence de la topographie sur la diffraction des ondes. D'autre part, dans presque tous les travaux cités ci-dessus, le comportement du sol a été considéré linéaire. Seuls, JOYNER (1975) et JOYNER et CHEN (1975) ont effectué des calculs en ayant pris en compte un comportement anélastique très simplifié du sol.

Pour étudier la réponse d'un sol à une sollicitation sismique, il faut connaitre l'évolution spatiale du champ incident sismique, et cela n'est possible que si la source est intégrée dans la modélisation du problème (BOU. CHON, 1973). L'intégration de la source est théoriquement possible, mais impose des couts de calcul prohibitifs surtout lorsque le foyer du séisme est loin de la zone étudiée. C'est pourquoi, en pratique, on suppose connu le champ seulement en quelques points appelés les points de contrôle. Cependant, sauf dans certains cas particuliers, la connaissance d'accélérogrammes en quel. ques points est insuffisante pour décrire complètement le champ incident sismique. On sera donc amené à imposer des conditions supplémentaires sur la nature du champ incident pour connaitre les conditions aux limites sur la frontière du maillage dans un calcul en éléments finis.

Une méthode très couramment utilisée en génie parasismique, consiste à évaluer la réponse du sol soumis à une onde $\mathrm{P}$ ou $\mathrm{SH}$ à propagation verticale. Cette approche n'est valable que si le foyer est très profond et les caractéristiques des matériaux, constituant le trajet de londe montante vers la surface du sol sont de telle sorte, que la vitesse de propagation diminue progressivernent en approchant la surface. Cette hypothèse simplificatrice conduit à un mouvement identique en chaque point de la surface de sol, dans le cas d'une stratigraphie horizontale. Il s'avère parfois indispensable de prendre en compte les ondes à propagation non verticale. LUCO (1980) propose pour cela, un modèle dans lequel le champ sismique incident est constitué de trois composantes de déplacement

- un déplacement horizontal perpendiculaire à la direction épicentre-site, modélisé par une onde plane $\mathrm{SH}$ à propagation non verticale, représente la contribution des ondes $\mathrm{SH}$ et de Love;

- une deuxième composante horizontale et une composante verticale modélisées par une onde plane SV et une onde plane $\mathrm{P}$ à propagation non verticale, représentent la contribution des ondes P, SV et de Rayleigh.

Pour connaître l'incidence de l'onde sismique, il faut évaluer la direction épicentre-site. L'amplitude des composantes du déplacement est proportionnelle aux moments sismiques qui modélisent la source, et il faut des hypothèses supplémentaires pour poursuivre son évolution. La présentation du champ sismique incident sous forme d'ondes proposée par LUCO, s'applique au champ lointain, pour lequel des vitesses de phase équivalentes peuvent être définies de façon approchée. Pour les champs proches on peut envisager suivant la nature du séisme une modélisation indépendante qui fournit les données nécessaires pour la modélisation du comportement des couches superficielles.

\section{Frontières absorbantes}

Très souvent en géotechnique, le domaine de sol à étudier au voisinage de la structure est de très grande dimension, par rapport à celle-ci, et doit être modélisé comme un milieu infini. Cela crée des difficultés numériques pour une méthode telle que la méthode des éléments finis car des réflexions d'ondes parasites se produisent à la frontière du maillage. Une technique de frontière absorbante, basée sur l'approximation paraxiale est présentée dans ce travail.

Moyennant une hypothèse de linéarité du comportement du sol, plusieurs méthodes ont été jusqu'à maintenant présentées dans la littérature pour modéliser le domaine extérieur non borné. Pour les problèmes stationnaires, les frontières consistantes permettent d'obtenir un opérateur impédance adapté pour toutes les ondes incidentes. Malheureusement les frontières ainsi conçues sont dépendantes de la fréquence et leur caractère non local conduirait à des calculs assez lourds dans le domaine transitoire.

Pour le cas transitoire, on peut construire des frontières absorbantes locales en espace et en temps à l'aide d'un certain nombre d'hypothèses. La première tentative pour construire ces frontières locales est due à LYSMER et KUHLEMEYER (1969) qui ont proposé des amortisseurs nodaux, très utilisés en pratique jusqu'à maintenant dans les modélisations numériques des problèmes dynamiques. Ces amortisseurs sont faciles à implanter dans un code de calcul, et ils fonctionnent très bien pour les ondes 
incidentes normales à la frontière mais ils présentent un certain nombre d'inconvénients si l'onde incidente est une onde de Rayleigh ou si l'onde sortante est légèrement inclinée.

L'approximation paraxiale ou parabolique (ENGQUIST et MAJDA, 1977, et CLAYTON et ENGQUIST, 1977). pour les ondes élastiques, présente un outil intéressant pour les modélisations dans le domaine transitoire et permet de construire une impédance dynamique sur l'interface $\Sigma$ qui soit locale en espace et en temps. COHEN et JENNINGS (1983) proposent une modélisation numérique de cette approximation par la méthode des éléments finis. C'est cette méthode que nous présenterons rapidement plus loin.

Après cette rapide revue bibliographique, incomplète car les sujets abordés sont très vastes, il est possible de présenter les différents points qui seront abordés dans ce travail :

1. Quelques renseignements fournis par l'homogénéisation :

2. Un modèle macroscopique de Biot ;

3. Aspects du comportement non élastique cyclique des sols ;

4. Modélisation numérique en dynamique des sols :

5. Applications au génie parasismique.

\section{QUELQUES RENSEIGNEMENTS FOURNIS PAR L'HOMOGÉNÉISATION DES MILIEUX POREUX BIPHASIQUES}

Le comportement des milieux poreux, à l'échelle macroscopique ne peut pas être décrit sans difficulté par les concepts classiques de la mécanique des milieux continus, car il dépend de la structure microscopique du matériau. Depuis quelques années, il est le sujet de diverses études utilisant des approches différentes. Dans toutes les méthodes d'homogénéisation la démarche est la suivante: après avoir décrit le comportement du matériau qu'on désire étudier au niveau microscopique en supposant cette fois que la mécanique des milieux continus est applicable aux différents composants du milieu et à leurs interactions, on passe au niveau macroscopique, et c'est ce passage qui peut être différent d'une méthode à une autre.

Les deux méthodes d'homogénéisation les plus couramment utilisées sont la Méthode des Moyennes et la Méthode des Développernents Asymptotiques, qui au prix d'une hypothèse supplémentaire de périodicité fournit les renseignements quantitatifs les plus précis. Elles utilisent toutes deux le concept de double échelle: l'échelle microscopique définie afin de représenter la structure interne du milieu poreux et l'échelle macroscopique définie au niveau de la structure que l'on souhaite modéliser. La notion de Volume Elémentaire Représentatif (VER) qui correspond à une échelle intermédiaire dans laquelle l'hétérogénéité du matériau est observable est un outil essentiel dans cette approche. La dimension du VER, au sein de la structure, doit être assez grande par rapport aux hétérogénéités locales du sol permettant de donner aux grandeurs moyennes (e.g. porosité, contraintes...) une signification sans ambiguité, mais petite par rapport aux dimensions de la structure.
Dans le calcul d'un ouvrage réel la dimension du VER peut être assimilée à la taille d'un échantillon de laboratoire. Dans les paragraphes qui vont suivre, seuls les aspects qualitatifs les plus importants seront rappelés renvoyant à la littérature pour plus de détails (BEAR, 1984 : AURIAULT, 1984 : MODARESSI, 1987).

Soient alors, $\sigma$ le tenseur des contraintes, $\epsilon$ le tenseur des déformations, u le vecteur déplacement, D le tenseur d'élasticité, $\rho$ la masse volumique microscopique, $\mu$ la viscosité du fluide, $\mathrm{p}$ la pression du fluide, $\mathrm{n}$ le vecteur unitaire normale extérieure. Les indices $s$ et $f$ se rapportent respectivement à la phase solide et la phase fluide. Les hypothèses suivantes sont retenues ; approximation des petites déformations pour la partie solide, le fluide considéré en écoulement lent et termes de convection négligés.

Le comportement de chaque phase au niveau microscopique est décrit à l'aide de la mécanique des milieux continus. On considère un solide élastique linéaire occupant dans le VER le domaine $\Omega_{s}$ et un fluide visqueux newtonien occupant le domaine $\Omega_{f}$. Le passage micromacro évoqué dans la section précédente se traduit en prenant les moyennes simples ou pondérées des quantités microscopiques (AURIAULT et SANCHEZ PALENCIA, 1977 ; BEAR, 1984). Ici seule la méthode des moyennes simple sans hypothèse de périodicité est présentée. Cette méthode n'est applicable que sur les milieux faiblement hétérogènes où les interactions entre les $\mathrm{VER}_{\mathrm{s}}$ peuvent être négligées. La méthode des développements asymptotiques liée à une hypothèse de périodicité ne sera pas rappelée (BORNE, 1983).

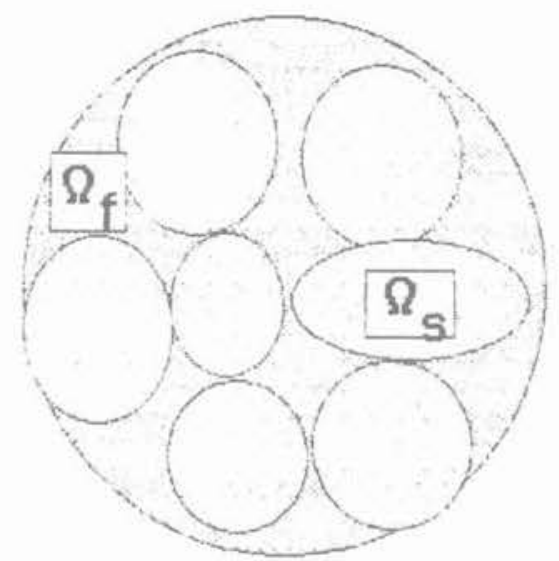

Fig. 1. - Schéma d'un Volume Elémentaire Représentatif. Fig. 1. - Schematic presentation of a representative elementary volume.

Soit $\Omega$ le milieu microscopique, $\Omega_{\alpha}(\alpha=s$, f $)$ une des deux phases constituant ce milieu et $\mathrm{S}_{\alpha \beta}$ l'interface entre les phases $\alpha$ et $\beta$, pour une fonction $\phi$ quelconque on définit respectivement, en notant $|\Omega|$ le volume microscopique et $\left|\Omega_{a}\right|$ le volume de chaque composant, la moyenne, la moyenne apparente et la moyenne vraie :

$$
\begin{aligned}
& \langle\phi\rangle=1 /\left.|\Omega|\right|_{\Omega} \phi \mathrm{dV} \\
& \langle\phi\rangle_{\alpha}=1 /|\Omega| \Omega_{\alpha} \phi \mathrm{dV} \\
& \langle\phi\rangle^{\alpha}=1 /\left.\left|\Omega_{\alpha}\right|\right|_{\Omega_{\alpha}} \phi \mathrm{dV} .
\end{aligned}
$$


On définit également la fraction volumique par:

$\theta_{\alpha}=\left|\Omega_{a}\right| /|\Omega|$.

Le passage des équations écrites au niveau microscopique aux équations macroscopiques nécessite les formules suivantes (BEAR et PINDER, 1978) :

$$
\begin{aligned}
& \langle\operatorname{grad} \phi\rangle_{\alpha}=\operatorname{grad}\langle\phi\rangle_{\alpha}+\left(1 /|\Omega| \mathrm{S}_{\alpha} \phi \mathrm{n}_{\alpha} \mathrm{ds}\right. \\
& \left\langle\partial_{\phi} \phi\right\rangle_{\alpha}=\partial_{t}\langle\phi\rangle_{\alpha}-(1 /|\Omega|) \mathrm{S}_{\alpha} \phi \partial_{1} \mathrm{u}_{\alpha} \cdot \mathrm{n}_{\alpha} \mathrm{ds}
\end{aligned}
$$

avec :

$$
\mathrm{S}_{\alpha}=U_{\beta} \Gamma_{\alpha \beta} \quad \alpha \neq \beta
$$

\subsection{Conservation de la quantité de mouvement macroscopique}

La méthode des moyennes conduit au résultat suivant. où les crochets $\langle>$ désignent des moyennes définies sur les phases solide ou fluide:

$\operatorname{Div}\left\langle\sigma_{s}\right\rangle_{s}+\left\langle\rho_{s}\right\rangle_{s} \mathrm{~g}+1 / \Omega \mathrm{S}_{\mathrm{s}} \sigma_{s} \cdot \mathrm{n}_{\mathrm{s}}=\left\langle\rho_{s} \partial_{t t} \mathrm{u}_{\mathrm{s}}\right\rangle$,

$\operatorname{Div}\left\langle\sigma_{\mathrm{f}}\right\rangle_{\mathrm{f}}+\left\langle\rho_{\mathrm{f}}\right\rangle_{\mathrm{f}} \mathrm{g}+1 / \Omega \mathrm{S}_{\mathrm{f}} \sigma_{\mathrm{f}} \cdot \mathrm{n}_{\mathrm{f}}=\left\langle\rho_{\mathrm{f}} \partial_{n} \mathrm{u}_{\mathrm{f}}\right\rangle_{\mathrm{f}}$

Si on appelle $\mathrm{n}$ la porosité du squelette solide, les fractions volumiques $\theta_{5}$ et $\theta_{1}$ s'écrivent :

$$
\theta_{s}=(1-n) . \quad \theta_{1}=n
$$

La contrainte totale est alors donnée sous la forme suivante :

$$
\begin{aligned}
\langle\sigma\rangle & =\left\langle\sigma_{s}\right\rangle_{s}+\left\langle\sigma_{i}\right\rangle_{t}=\theta_{s}\langle\sigma\rangle^{s} \\
& -\theta_{f}\langle\mathrm{p}\rangle^{\prime} \mathrm{I}+\theta_{\mathrm{i}}\left\langle\tau_{\mathrm{f}}\right\rangle^{\prime}
\end{aligned}
$$

en décomposant les contraintes dans le fluide en partie isotrope et déviatoire. En mécanique des sols, la pression mesurée étant la pression p dans la phase fluide, nous posons :

$$
\mathrm{p}=\langle\mathrm{p}\rangle^{1}
$$

Suivant le principe de la contrainte effective de Terzaghi, celle-ci est définie par:

$$
\left\langle\sigma^{\prime}\right\rangle=\left\langle\sigma^{\prime}\right\rangle+\mathrm{p} \mathrm{I}
$$

Le terme correspondant à la contrainte effective s'exprime par la relation suivante :

$$
\left\langle\sigma^{\prime}\right\rangle=\left\langle\sigma_{s}\right\rangle_{\mathrm{s}}+(1-\mathrm{n}) \mathrm{p} \mathrm{I}+\mathrm{n}\left\langle\tau_{\mathrm{f}}\right\rangle^{f}
$$

qui montre l'influence que la pression interstitielle peut avoir sur la phase solide, ainsi que la contrainte déviatoire, due à la viscosité de l'eau. En général, en mécanique des sols. ces deux termes sont négligés. Enfin, la masse volumique totale s'écrit en fonction des masses volumiques de chacune des phases :

$$
\begin{aligned}
\langle\rho\rangle & =\left\langle\rho_{s}\right\rangle_{s}+\left\langle\rho_{s}\right\rangle_{1} \\
& =(1-n)\left\langle\rho_{s}\right\rangle^{s}+n\left\langle\rho_{1}\right\rangle^{1} .
\end{aligned}
$$

On obtient alors l'équation d'équilibre macroscopique globale en contrainte totale en ajoutant les deux équations dynamiques :

$\operatorname{Div}\langle\sigma\rangle+\langle\rho\rangle g=\left\langle\rho_{s} \partial_{\mathrm{t}} \mathrm{u}_{\mathrm{s}}\right\rangle+\left\langle\rho_{\mathrm{f}} \partial_{\mathrm{u}} \mathrm{u}_{\mathrm{f}}\right\rangle$.
Cette équation peut également s'écrire en fonction des contraintes effectives:

Div $\langle\sigma\rangle-\operatorname{grad} \mathrm{p}+\langle\rho\rangle \mathrm{g}=\left\langle\rho_{\mathrm{g}} \partial_{\mathrm{H}} \mathrm{u}_{\mathrm{s}}\right\rangle+\left\langle\rho_{\mathrm{f}} \partial_{\mathrm{H}} \mathrm{u}_{\mathrm{i}}\right\rangle$.

On voit apparaître ici le rôle de l'eau dans le comportement dynamique, en particulier par la participation des pressions interstitielles à l'équilibre dynamique du milieu poreux.

\subsection{Loi de Darcy généralisée}

Pour obtenir la loi de Darcy généralisée, qui est une loi macroscopique, il faut connaitre le terme:

$$
\mathrm{R}=1 / \Omega \mathrm{S}_{\mathrm{f}} \sigma_{\mathrm{i}} \cdot \mathrm{n}_{\mathrm{f}}
$$

qui se manifeste dans l'équation de la dynamique de la phase fluide et le relier ensuite à la vitesse de filtration :

$$
<\partial_{t} u_{1}-\partial_{t} u_{s}>_{i}
$$

A priori il n'y a pas de relation univoque entre ces deux quantités et il faut des hypothèses supplémentaires. On fait généralement l'hypothèse de linéarité entre la vitesse relative du fluide et le terme $\mathrm{R}$ en faisant apparaitre la perméabilité microscopique $\mathrm{k}$ en tant qu'opérateur de localisation :

$$
\left\langle\partial_{t} u_{f}-\partial_{t} u_{s}\right\rangle_{t}=k \cdot R
$$

d'où grâce à l'équation de conservation de la quantité de mouvement dans la phase fluide:

$$
\mathrm{R}=\left\langle\rho_{1} \partial_{1 t} u_{1}-\operatorname{Div} \mathrm{n}\left\langle\sigma_{1}\right\rangle^{\prime}-\mathrm{n}\left\langle\rho_{1}\right\rangle^{\prime} \mathrm{g} .\right.
$$

Dans la littérature, le premier terme du membre de droite de cette dernière relation est approchée par :

$$
\left\langle\rho_{i} \partial_{t 1} u_{i}\right\rangle_{i} \approx n\left\langle\rho_{i}\right\rangle^{l}\left\langle\partial_{t 1} u_{i}\right\rangle_{i} .
$$

Si bien que la vitesse moyenne relative du fluide par rapport au solide $\partial_{1} u_{1 f}$ sera donnée par :

$$
\begin{aligned}
\left\langle\partial_{1} u_{n f}\right. & =\left\langle\partial_{1} u_{i}-\partial_{1} u_{s}\right\rangle^{t} \\
& =k \operatorname{grad}\left(p-\left\langle\rho_{1}\right\rangle^{\prime} g x\right) \\
& +\left\langle\rho^{\prime}\right\rangle^{t}\left\langle\partial_{t t} u_{i}\right\rangle_{i}-\operatorname{Div}\left\langle\tau_{1}\right\rangle^{\prime}
\end{aligned}
$$

\subsection{Conservation de la masse globale}

En moyennant les équations locales de conservation de la masse, on en déduit pour la phase solide :

$$
\begin{aligned}
& (1-n) \partial_{t}\left\langle\rho_{s}\right\rangle^{s} /\left\langle\rho_{s}\right\rangle_{s} \\
& \quad+\operatorname{div}\left((1-n)\left\langle\partial_{s} u_{s}\right\rangle^{s}\right)-\partial_{t} n=0
\end{aligned}
$$

et pour la phase fluide:

$\left.n \partial_{1}\left\langle\rho_{f}\right\rangle^{\prime} /\left\langle\rho_{f}\right\rangle^{\prime}+\operatorname{div}\left(n<\partial_{l} u_{i}\right\rangle^{\prime}\right)+\partial_{1} n=0$.

L'équation de la conservation de masse pour l'ensemble des deux phases solide et fluide s'écrit alors:

$$
\begin{aligned}
& (1-n) \partial_{1}\left\langle\rho_{s}\right\rangle^{5} /\left\langle\rho_{s}\right\rangle^{s}+n \partial_{t}\left\langle\rho_{f}\right\rangle^{f} /\left\langle\rho_{f}\right\rangle^{1} \\
& \left.\left.+\operatorname{div}(1-n)<\partial_{t} u_{s}\right\rangle^{3}+n<\partial_{i} u_{f}\right\rangle^{i}=0 \text {. }
\end{aligned}
$$


$\mathrm{K}_{\mathrm{s}}$ et $\mathrm{K}_{\mathrm{f}}$ étant les modules de compressibilité de la matrice solide et du fluide interstitiel, on obtient la relation suivante exprimée en vitesse de filtration du fluide

$$
\begin{gathered}
-(1-n) \operatorname{Tr}\left\langle\partial_{1} \sigma_{s}\right\rangle^{s} / K_{s}-n \operatorname{Tr}\left\langle\partial_{s} \sigma_{i}\right\rangle^{i} / K_{4} \\
+\operatorname{div}\left(\left\langle\partial_{1} u_{s}\right\rangle+\left\langle\partial_{t} u_{n}\right\rangle\right)=0 .
\end{gathered}
$$

La dépendance fréquentielle de la perméabilité, lorsqu'on utilise la méthode des développements asymptotiques, permet de mettre en évidence le couplage inertiel fonction de la fréquence (BORNE, 1983) tandis que dans la méthode des moyennes si le couplage inertiel existe, il est indépendant de la fréquence. Ce dernier point est important lorsqu'on veut modéliser le comportement d'un milieu poreux dans le domaine des basses fréquences. car dans ce cas, le tenseur de perméabilité dans la méthode des développements asymptotiques pouvant être considéré indépendant de la fréquence, nous trouvons immédiatement une relation dans laquelle seulement l'inertie de la phase solide se présente. Dans la méthode des moyennes, on sera amené à éliminer l'accélération relative du fluide par rapport au squelette solide sachant a priori que ce terme ne joue un rôle faible à basse fréquence.

Enfin le terme Div $\left\langle\tau_{i}\right\rangle^{\dagger}$ dans l'équation de la dynamique représentant le comportement visqueux du fluide au niveau macroscopique peut être éliminé lorsque le fluide interstitiel est de l'eau. Dans la méthode des développements asymptotiques la viscosité du fluide interstitiel au niveau macroscopique est négligée dans le choix de l'ordre de grandeur de la contrainte visqueuse par rapport à la pression.

Dans le paragraphe suivant nous présenterons l'approche macroscopique de Biot pour modéliser les milieux poreux biphasiques et saturés. Nous comparerons ensuite les formulations présentes ici prenant en compte la structure microscopique du milieu avec celles de Biot fondée sur les observations purement macroscopiques.

\section{UN MODÈLE MACROSCOPIQUE DE BIOT}

Dans le présent article, seule la formulation dite simplifiée est présentée, renvoyant à la thèse de $\mathrm{H}$. MODA RESSI (1987) pour la présentation du modèle complet. La phase fluide au sein de laquelle se développe l'écoulement est continue, et la matrice est constituée d'une phase solide et éventuellement de pores occlus. L'influence des pores non connectés peut être éventuellement prise en compte dans la compressibilité du squelette solide, de la même façon le rôle de l'air dissous dans le fluide peut être introduit par augmentation de la compressibilité du fluide. La dernière hypothèse porte sur la longueur d'onde supposée grande, devant la dimension des pores. Si la longueur d'onde est grande devant le volume élémentaire, la perméabilité peut être considérée indépendante de la fréquence (modèle des basses fréquences de Biot). En supposant la perméabilité indépendante de la fréquence et en négligeant le couplage inertiel, on retrouve cette formulation simplifiée de l'équation hydraulique. Cette indépendance fré- quentielle à basses fréquences a été également proposée par BIOT.

\subsection{Conservation de la quantité de mouvement globale}

La conservation de la quantité de mouvement globale en contrainte totale et en présence des forces de volume et inertielles s'écrit alors, en négligeant l'accélération relative du fluide:

$$
\text { Div } \sigma^{\prime \prime}+\rho \mathrm{g}=\rho \partial_{n} \mathrm{u}_{\mathrm{s}}
$$

alors qu'en terme de contrainte intergranulaire, avec $\alpha$ désignant un coefficient fonction de la compressibilité de la matrice solide, on obtient :

$$
\text { Div } \sigma^{\prime \prime}-\alpha \operatorname{grad} p+\rho \mathrm{g}=\rho \partial_{n} \mathrm{u}_{s}
$$

\subsection{Loi de Darcy généralisée}

La loi de Darcy généralisée, dans le cadre du modèle simplifié, est donnée par :

$$
\partial_{1} u_{r f}=K\left(-\operatorname{grad} p+\rho_{f} g-\rho_{i} \partial_{n} u_{s}\right)
$$

\subsection{Conservation de la masse globale}

Les équations de conservation de la masse, pour chaque phase, conduisent à l'équation suivante :

$\operatorname{div} \partial_{t} u_{r}+\alpha \operatorname{div} \partial_{t} u_{s}=-n \partial_{t} p / K_{f}-(\alpha-n) \partial_{t} p / K_{s}$

Intégrant l'équation ci-dessus par rapport au temps seulement, il est alors possible d'éliminer $\partial_{t} \mathrm{u}_{\mathrm{rf}}$ dans l'équation de Darcy, ce qui permet d'écrire le problème en fonction des variables $u_{\varepsilon}$ et $p$, uniquement :

$\alpha \operatorname{div} \partial_{1} \mathrm{u}_{\mathrm{s}}-\operatorname{div}\left(\mathrm{K} \operatorname{grad}\left(\mathrm{p}-\rho_{\mathrm{g}} \mathrm{x}\right)\right)$

$$
=-\partial_{t} \mathrm{p} / \mathrm{Q}+\operatorname{div}\left(K \rho_{i} \partial_{t t} u_{5}\right)
$$

Dans l'équation le terme div $\left(\mathrm{K} \rho_{t} \partial_{\mathrm{t}} \mathrm{u}_{\mathrm{s}}\right)$ introduit des matrices asymétriques et nous verrons dans les formulations numériques comment traiter ce problème. Le domaine d'applicabilité du modèle simplifié sera discuté plus loin.

\subsection{Conditions aux limites}

Les conditions aux limites macroscopiques sont rarement discutées dans les articles traitant de l'homogénéisation. Il va sans dire qu'elles sont indispensables à la modélisation numérique. Les conditions aux limites, particulières à la prise en compte du champ sismique libre seront discutées dans la section 5 . Il convient de distinguer les conditions aux limites mécaniques et hydrauliques.

\section{a. Conditions aux limites mécaniques}

Les conditions aux limites mécaniques du modèle simplifié, sont de deux types : soit en déplacements, soit en forces. On écrit les conditions aux limites, pour l'équilibre global, en partitionnant la frontière $\Gamma$ du domaine $\Omega$ en deux parties complémentaires:

$$
\Gamma=\Gamma_{o} \cup \Gamma_{\text {us }}
$$


Sur la partie $\Gamma_{u s}$ de la frontière, une condition aux limites en déplacements est imposée:

$$
\mathrm{u}_{s}=\mathrm{u}_{\mathrm{s}}^{*}, \quad \mathrm{x} \in \Gamma_{\mathrm{us}}
$$

Le déplacement impose $\mathrm{u}_{\mathrm{s}}^{*}$ est souvent nul en pratique, par exemple à l'interface avec le substratum

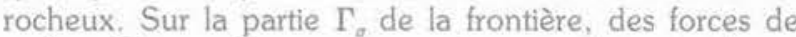
surface $T$ imposées au vecteur des contraintes totales et non au vecteur des contraintes intergranulaires ou effectives sont imposées:

$$
\sigma \cdot \mathrm{n}=\mathrm{T}, \quad \mathrm{x} \in \Gamma_{\sigma}
$$

Ceci est important du point de vue de la formulation variationnelle correcte, en vue de l'approximation numérique.

\section{b. Conditions aux limites hydrauliques}

Les conditions aux limites hydrauliques sont également de deux types : flux imposés ou pressions imposées et de même, elles s'écrivent respectivement en considérant une autre partition de la frontière $\Gamma$ :

$\Gamma=\Gamma_{p} \cup \Gamma_{\varphi}$

$\mathrm{p}=\mathrm{p}^{*}, \quad \mathrm{x} \in \Gamma_{\mathrm{p}}$

$\partial_{1} u_{f i} \cdot \mathrm{n}=\varphi, x \in \Gamma_{\varphi}$.

Là encore, les conditions aux limites sont adaptées à la bonne formulation variationnelle du problème numérique.

\subsection{Loi de comportement élastoplastique en contraintes effectives}

Celle-ci sera détaillée plus loin, dans la section portant sur le comportement cyclique des sols, mais nous en retiendrons la forme symbolique suivante, qui dans le cadre de toute formulation élastoplastique donne le taux de contraintes effectives et de variables d'écrouissage $\alpha$ en fonction du taux de déformation :

$\partial_{1} \sigma^{\prime \prime}=D^{e p} \partial_{1} \epsilon$

$\partial_{1} \alpha=L \partial_{t} \epsilon$

par l'intermédiaire de la matrice élastoplastique $D^{\text {ep }}$ et de la loi d'évolution L.

Outre les conditions aux limites, des conditions initiales doivent porter sur les déplacements, contraintes effectives, variables d'écrouissage et pression interstitielle.

On peut finalement résumer le problème à résoudre, dans le cas du modèle simplifié, de la manière suivante:

Problème couplé dynamique : chercher des champs de déplacement $\mathrm{u}_{4}(\mathrm{x}, \mathrm{t})$, de pression $\mathrm{p}(\mathrm{x}, \mathrm{t})$ et de contraintes et variables d'écrouissage $\left(\sigma \sigma^{\prime \prime}(\mathrm{x}, \mathrm{t}), \alpha(\mathrm{x}, \mathrm{t})\right)$ qui satisfont aux équations précédentes et qui vérifient les conditions aux limites; les conditions initiales $u_{s}(x, 0), p$ $(x, 0), \partial_{1} u_{s}(x, 0),\left(\sigma^{\prime \prime}(x, 0), \alpha(x, 0)\right)$ étant connues.

\subsection{Domaine d'application du modèle}

Dans la formulation simplifiée, l'accélération relative du fluide devant l'accélération du squelette pour les problèmes à basses fréquences est négligée. Il faut noter que cette hypothèse est vérifiée pour l'équation hydraulique dans la méthode d'homogénéisation bâtie à partir des développements asymptotiques. Pour les problèmes à fréquences plus élevées, par lapproche par homogénéisation et hypothèse de périodicité, intervient une dépendance fréquentielle des termes de couplages visqueux et inertiels alors que BIOT prend en compte seulement la dépendance fréquentielle des termes visqueux (BORNE, 1983).

Une fréquence dite caractéristique sépare les deux domaines de basses et hautes fréquences. La définition de cette fréquence caractéristique ne peut se faire qu'à partir de la physique du problème et correspond à la limite de l'écoulement du type Poiseuille. Une approche (GELI, 1985) consiste à supposer les forces inertielles négligeables devant les forces de couplage visqueuses, ce qui permet d'obtenir l'expression suivante :

$$
\mathrm{f}_{c} \approx \mathrm{g} /(2 \pi \mathrm{k})
$$

avec $\mathrm{k}$ la perméabilité cinématique $\left(\mathrm{k}=\mathrm{g} \mathrm{k}^{*} / \mathrm{V}^{*}, \mathrm{k}^{*}\right.$ la perméabilité géométrique et $\mathrm{V}^{*}$ la viscosité cinématique du fluide). La fréquence caractéristique peut être également déterminée en fonction de la taille des pores. On cherche alors la taille maximale (d) à partir de laquelle l'écoulement à l'intérieur du pore ne peut pas être considéré comme un écoulement du type Poiseuille. Il faut rappeler que dans la formulation simplifiée $\left(\mathrm{u}_{\mathrm{s}}-\right.$ p) l'hypothèse prise est plus forte que celle des basses fréquences, car on néglige en outre, l'accélération relative du fluide dans l'équation d'équilibre global.

Pour évaluer le domaine d'application de chacune des formulations, ZIENKIEWICZ et al. (1984) ont effectué une étude monodimensionnelle dans laquelle une charge périodique est appliquée sur la surface. On permet le drainage seulement en haut de la colonne. La perméabilité est supposée constante. Le fluide est parfait et le comportement du squelette est supposé élastique linéaire, ce qui permet de résoudre facilement le problème en effectuant une transformation de Fourier par rapport au temps. En négligeant la compressibilité des grains, ces auteurs aboutissent à délimiter les domaines d'application de chaque formulation présentés dans la figure 2 à l'aide des paramètres adimensionnels $\Pi_{1}$ et $\Pi_{2}$;

$$
\Pi_{1}=c_{v} T / 2 \pi L^{2} \quad \Pi_{2}=\omega L / c_{p}=a_{0}
$$

où, $\mathrm{L}$ représente la hauteur de la colonne, $c_{p}$ la vitesse de propagation des ondes $\mathrm{P}$ dans le milieu homogène équivalent, $\omega$ la fréquence angulaire de la charge appliquée, $\mathrm{T}$ la période correspondante, et $\mathrm{K}$ la perméabilité $\left(=k / \rho_{i} \mathrm{~g}\right)$. Les termes $c_{v}$ et $a_{0}$ sont respectivement les coefficients de consolidation classiques de la mécanique des sols et la fréquence adimensionnelle utilisée en interaction dynamique sol-structure. Le terme $\Pi_{1}$ est donc (à $2 \pi$ près) le temps réduit de la théorie de la consolidation classique et $\Pi_{2}$ exactement la fréquence réduite. Par conséquent $\Pi_{1}$ caractérise globalement l'aspect mono ou biphasique du mélange: si $\Pi_{1}$ est petit (cas dit « non drainé » en mécanique des sols) le mélange se comporte essentiellement comme un milieu 
monophasique. $\Pi_{2}$ est plutôt relatif à la longueur d'onde caractéristique par rapport à la taille du domaine. Dans cette figure (fig. 2), on distinguera :

- la zone 1: phénomène lent (accélérations des deux phases négligeables) ;

- la zone 2 : vitesse modérée (accélération relative de la phase fluide négligeable);

- la zone 3 : phénomène rapide (modèle complet).

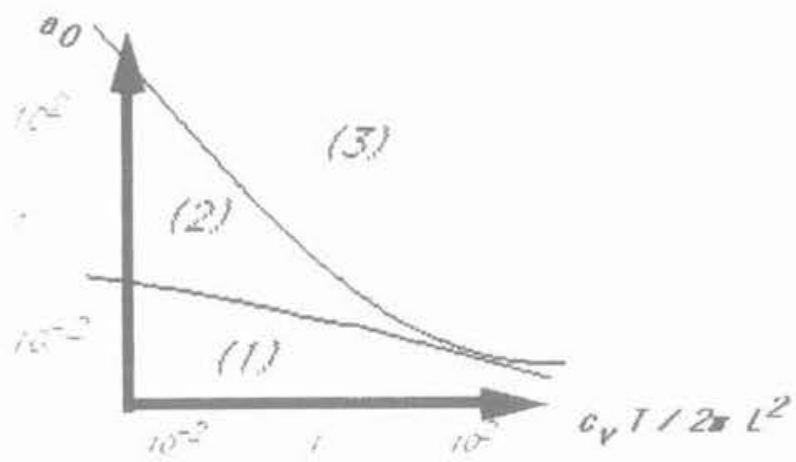

Fig. 2. - Domaine d'application de chaque modéle (d'après Zienkeiwicz et al. (1980)).

Fig. 2. - Domain of application of each model (following 2...)

On peut constater que le produit $\Pi_{1}$. $\left(\Pi_{2}\right)^{2}$ correspondant à la limite d'applicabilité de la formulation simplifiée est approximativement égal à l'unité ; or si nous calculons la valeur de la fréquence correspondante nous obtenons :

$$
f \approx \rho_{f} g /(2 \pi \rho k)
$$

On s'aperçoit alors que la fréquence caractéristique semble une expression acceptable (dans le cadre des hypothèses faites) donnant la limite supérieure de l'applicabilité de la formulation simplifiée. Dans certains sols très perméables $\left(\mathrm{k}=10^{-1}\right.$ à $\left.10^{-2} \mathrm{~m} / \mathrm{sec}\right)$, la fréquence caractéristique peut être dans la plage habituelle des fréquences sismiques (inférieures à environ $20 \mathrm{Hertz}$ ). Pour les valeurs de $\Pi_{1}$ supérieures à $10^{2}$, le problème peut être considéré drainé, car le chargement est très lent et la pression interstitielle dans le fluide a le temps de se dissiper. Pour les chargements rapides $\left(\Pi_{1}\right.$ très faible et $\Pi_{2}$ grand) le comportement du sol est un comportement non drainé. On peut alors éventuellement envisager des calculs monophasiques mais en prenant en compte des paramètres non drainés pour définir le comportement de l'ensemble. Le problème qui se pose est qu'au voisinage des endroits où un drainage peut s'effectuer se développe une couche limite dans laquelle, quelle que soit la fréquence de chargement, une dissipation de la pression interstitielle s'effectue.

\section{COMPORTEMENT ÉLASTOPLASTIQUE CYCLIQUE DES SOLS}

Le sol a un comportement fortement non linéaire et cette non-linéarité se manifeste dès le début du chargement déviatoire ou isotrope. Les aspects qualitatifs sui- vants sont importants, du point de vue de la modélisation, dès lors que dans un calcul de propagation d'ondes existeront des zones subissant des déformations couvrant une large gamme :

- les résultats expérimentaux sur les sols (ISHIHARA, 1982 : HUJEUX, 1985 et HICHER, 1985) montrent que, lorsque l'amplitude des déformations cycliques de cisaillement $\gamma$ c est inférieur à $10^{-5}$, le comportement des sols reste élastique mais non linéaire car dépendant de la pression moyenne;

- lorsque $\gamma_{c}$ varie entre $10^{-5}$ et $10^{-4}$, le sol est dans un domaine hystérétique stabilisé, c'est-à-dire que la forme des cycles ne varie pas, si on continue le chargement cyclique. L'aire du cycle correspond à l'énergie dissipée pour créer la déformation plastique. L'analyse linéaire-équivalente (SEED et IDRISS, 1969) est applicable dans ce domaine. Rappelons que dans cette méthode on remplace le sol par un matériau linéaire avec amortissement de telle sorte que la rigidité et l'énergie dissipée entre les deux systèmes soient équivalentes. Il faut noter que la limite $10^{-4}$ est une limite approximative et sert seulement dans la modélisation numérique d'un problème particulier :

- pour les amplitudes de cycle $\gamma_{c}$ supérieures à $10^{-4}$ pour les sables on observe une modification de la forme des cycles, due à un réarrangement des grains qui, dans le cas drainé, provoque la densification du sol et dans le cas non drainé, augmente la pression interstitielle. Dans ce dernier cas cette augmentation entraîne la diminution de la contrainte effective et le phénomène de liquéfaction. L'évaluation de la pression interstitielle et de la déformation permanente, à ce niveau de déformation, ne peut se faire qu'à l'aide d'une loi de comportement incrémentale, c'est-à-dire, qui tienne compte de l'histoire du chargement, de l'état actuel de contraintes effectives et qui relie à chaque instant le tenseur des taux de contraintes effectives au tenseur des taux de déformations.

HUJEUX a présenté une lof de comportement élastoplastique multimécanisme avec écrouissage cinématique qui décrit le comportement des sols sous les chargements cycliques (AUBRY et al., 1982; HUJEUX, 1985). Trois mécanismes de déformations plastiques déviatoires en déformation plane dans trois plans orthogonaux sont introduits, ainsi qu'un mécanisme de consolidation purement volumique. Ces quatre mécanismes sont actifs aussi bien pendant les chargements primaires que cucliques. La décomposition se fait dans un repère fixe, et chaque mécanisme déviatoire sera équivalent à un critère de Mohr-Coulomb, relativement au plan correspondant. Les équations sont succinctement présentées dans les deux paragraphes suivants. La signification des paramètres est présentée ensuite.

\subsection{Modélisation du domaine élastique}

Les déformations élastiques sont définies par une loi incrémentale isotrope non linéaire. Le modèle choisi s'écrit pour la partie isotrope :

$$
\partial_{t} \epsilon_{V^{e}}=(1 / K) \partial_{t} p^{\prime}
$$

avec :

$$
K=K_{\text {réf }}\left(p^{\prime} / p_{\text {réf }}\right)^{n}
$$


et $\mathrm{p}_{\text {ré }}$ pression de référence, $\mathrm{K}_{\text {ré }}$ module volumique élastique de référence, $n$ exposant élastique, p' pression moyenne effective, $\epsilon_{\mathrm{v}}{ }^{\circ}$ déformation volumique élastique, $\mathrm{K}$ module volumique élastique. Une expression similaire est utilisée pour la partie déviatoire. Les paramètres $\mathrm{K}$ et $\mathrm{n}$ sont fonctions de la géométrie de l'assemblage, c'est-à-dire des variables d'écrouissage. Une analyse thermodynamique montre qu'en fait, il y a un couplage entre les parties isotrope et déviatoire, qui a été négligé ici.

\subsection{Modélisation du domaine plastique - chargement monotone}

Chaque mécanisme ou fonction de charge a une variable d'écrouissage, liée à la mobilisation du frottement. qui lui est propre alors que l'écrouissage en densité est commun. Dans les formules ci-dessous, l'indice $M$ se rapporte au chargement Monotone (soit le chargement primaire, soit un chargement à une intensité suffisante pour effacer l'histoire du chargement) et l'indice $C$ se rapporte au chargement cyclique.

\section{a. Fonction de charge monotone}

Elle est essentiellement représentée par le critère de Mohr-Coulomb, auquel on a ajouté un terme d'écrouissage $r_{a}$ appelé le taux de frottement mobilisé et une fonction $\mathrm{F}$ appelée fonction d'enchevêtrement:

$f^{\mathrm{M}} \mathrm{a}\left(\sigma^{\prime}, \mathrm{n}_{\mathrm{p}}, \mathrm{r}_{\mathrm{a}}\right)=$

$$
\sigma_{b}^{\prime}-\sigma_{c}^{\prime}+\left[\mathrm{F}\left(\sigma_{b}^{\prime}+\sigma_{c}^{\prime}, \mathrm{n}_{\mathrm{p}}\right) \mathrm{r}_{\mathrm{b}}\right]\left(\sigma_{\mathrm{b}}^{\prime}+\sigma_{\mathrm{c}}^{\prime}\right) \sin \phi
$$

où $\sigma_{\mathrm{b}}^{\prime}$ représente la contrainte principale effective numéro $\alpha \mathrm{b} \nsim, \phi$ l'angle de frottement de palier. La fonction $\mathrm{F}$ dépend du rapport : contrainte normale actuelle. contrainte d'enchevêtrement, sur ce plan, liée à la porosité plastique $n_{p}$ et est donnée par :

$\mathrm{F}\left(\sigma_{\mathrm{b}}^{\prime}+\sigma_{c}^{\prime}, \mathrm{n}_{\mathrm{p}}\right)=1-\mathrm{b} \log \left(\sigma_{\mathrm{b}}^{\prime}+\sigma_{\mathrm{c}}^{\prime} / \mathrm{p}_{\mathrm{c}}\left(\mathrm{n}_{\mathrm{p}}\right)\right)$ où $b$ est une constante du matériau et $p_{c}\left(n_{p}\right)$ représente la pression de consolidation donnée par:

$$
p_{c}\left(n_{p}\right)=p_{c o} \exp \left(-\beta n_{p}\right)
$$

L'intervention de la fonction F dans le critère de MohrCoulomb permet d'incorporer le concept d'état critique dans la description du comportement. On en déduit le taux de variation de la fonction $\mathrm{F}$, influencé à la fois par le taux de pression effective et de porosité :

$$
\mathrm{d}_{1} \mathrm{~F}=-\mathrm{b}\left(1 /\left(\sigma_{\mathrm{b}}^{\prime}+\sigma_{\mathrm{c}}^{\prime}\right)\right) \mathrm{d}_{\mathrm{t}}\left(\sigma_{\mathrm{b}}^{\prime}+\sigma_{\mathrm{c}}^{\prime}\right)+\beta \mathrm{d}_{\mathrm{t}} \mathrm{n}_{\mathrm{p}}
$$

De la même manière, on peut mettre en évidence, dans le taux de la fonction de charge $\mathrm{f}_{\mathrm{a}}$, c'est-à-dire la résistance instantanée du matériau, les termes dus au frottement et les termes dus à l'évolution des variables d'écrouissage :

$$
\begin{gathered}
\mathrm{d}_{1} \mathrm{f}_{\mathrm{a}}=\left(\partial_{a}\left(\sigma_{\mathrm{b}}+\sigma_{\mathrm{c}}^{\prime}\right)+\left(\mathrm{F}_{\mathrm{a}}-\mathrm{b}\right) \mathrm{r}_{\mathrm{a}} \sin \phi \partial_{\sigma}\left(\sigma_{\mathrm{b}}^{\prime}+\sigma_{\mathrm{c}}^{\prime}\right)\right\} \\
\mathrm{d}_{\mathrm{c}} \sigma+\left(\sigma_{\mathrm{b}}^{\prime}+\sigma_{\mathrm{c}}^{\prime}\right)\left(-\mathrm{b} \beta \mathrm{r}_{\mathrm{a}} \mathrm{d}_{1} \mathrm{n}_{\mathrm{p}}+\mathrm{F}_{\mathrm{a}} \mathrm{d}_{1} \mathrm{r}_{\mathrm{a}}\right) \sin \phi
\end{gathered}
$$

\section{b. Loi d'écoulement}

La loi d'écoulement relative à chaque mécanisme repose sur l'associativité par rapport à la différence des contrain- tes principales tout en respectant la loi de dilatance de Roscoe et l'état caractéristique défini par l'angle $\psi$. On en déduit que la direction des taux de déformations plastiques relatives au mécanisme $\alpha$ a $n$ est donnée par :

$$
\begin{aligned}
\psi_{\mathrm{a}} & =\partial_{\sigma}\left(\sigma_{\mathrm{b}}-\sigma_{\mathrm{c}}\right) \\
& +(1 / 3)\left(\sin \psi+\left(\sigma_{\mathrm{b}}-\sigma_{\mathrm{c}}\right) /\left(\sigma_{\mathrm{b}}+\sigma_{\mathrm{c}}\right)\right) 1
\end{aligned}
$$

La vitesse de déformation plastique totale est obtenue à partir de la contribution de chaque mécanisme pondérée par les multiplicateurs plastiques $\lambda^{p a}$ correspondants :

$$
D^{p}=\Sigma_{a} \lambda^{p a} \psi_{a}
$$

\section{c. Loi d'évolution des variables d'écrouissage}

L'évolution de la porosité plastique est obtenue, en utilisant la conservation de la masse à partir de la variation de volume plastique :

$$
d_{1} n_{p}=\Sigma_{b} \lambda^{p b} \operatorname{Tr}\left(D^{p b}\right)
$$

L'évolution du frottement mobilisé $\mathrm{r}_{\mathrm{a}}$ est donnée par une relation empirique très simple, où le paramètre a est supposé être une constante du matériau :

$$
\mathrm{d}_{\mathrm{r}} \mathrm{r}_{\mathrm{a}}=\lambda_{\mathrm{pa}}\left(1-\mathrm{r}_{\mathrm{a}}\right)^{2} / \mathrm{a}
$$

\section{d. Formulation élastoplastique}

Elle est tout à fait classique et permet de relier le taux de contraintes effectives au taux de déformations élastiques :

$\mathrm{d}_{1} \sigma^{\prime}=\lambda \operatorname{tr}(\mathrm{D}) 1+2 \mu \mathrm{D}-\Sigma_{\mathrm{b}} \lambda^{\mathrm{pb}} \lambda \operatorname{tr}\left(\mathrm{D}^{\mathrm{pb}}\right) \mathrm{I}+2 \mu \mathrm{D}^{\mathrm{pb}}$ où $\lambda$ et $\mu$ sont les paramètres élastiques de Lamé, obtenus à partir des modules définis dans le paragraphe précédent.

\subsection{Cas des chargements cycliques}

Le manque de place ne nous permet pas de développer les équations relatives au comportement cyclique et on reportera le lecteur à HUJEUX (1985). D'un point de vue qualitatif, celui-ci est caractérisé par une surface de charge relative à chaque mécanisme, possédant ses propres variables d'écrouissage évoluant continument entre deux charge-décharge ou décharge-charge consécutives, et remises à jour de manière discontinue lorsque de telles séquences se manifestent. Mises à part ces différences, les fonctions de charge cyclique sont semblables aux fonctions de charges monotones définies plus haut. La figure 3 donne une idée schématique de la configuration possible à un instant donné des surfaces de charge cycliques et monotones.

En outre la loi d'écoulement plastique cyclique tente de respecter les aspects expérimentaux qui ont été mentionnés plus haut, de la manière suivante. Dans le domaine cyclique-plastique, la loi de dilatance obtenue à partir de langle caractéristique est plus ou moins mobilisée suivant la valeur de la variable d'écrouissage $r_{a}$ : si $r_{a}$ est nul, il n'y a pas de variation de volume plastique et donc sur des chemins non drainés pas de variation de pression interstitielle; ce qui sera le cas évidemment dans le domaine élastique, mais aussi dans le domaine hysté- 


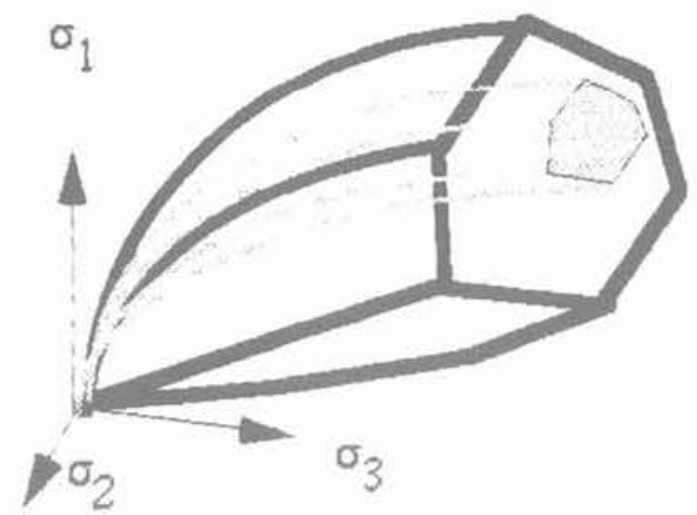

Fig. 3. - Surfaces de charge monotone et cyclique. Fig. 3. - Monotonic and cyclic yield surface.

rétique. Si $r_{\text {。 }}$ est plus important, la loi de dilatance de Roscoe généralisée est prise en compte, ce qui sera le cas au-delà du domaine mobilisé.

\subsection{Détermination des paramètres de la loi de comportement}

Le comportement des sols sous chargements cycliques est complexe et ne peut être mis en équation qu'à partir d'un nombre malheureusement assez grand de paramètres. La loi de Hujeux n'échappe pas à cette règle. Deux familles de paramètres interviennent : les paramètres physiques qui sont déterminés par les essais de laboratoire et les paramètres numériques propres à la formulation de la loi et qui servent à caler les courbes numériques et expérimentales. HUJEUX (1985) et HAJAL (1984) donnent un certain nombre de fourchettes des valeurs pour ces paramètres. Un effort est encore nécessaire pour compléter ces travaux pour définir des paramètres sur un plus grand nombre de sols. En outre, ces paramètres peuvent être classés en fonction de leurs natures et leurs domaines d'application : paramètres élastiques, d'état critique, d'écrouissage, et de limites de comportement.

La détermination des paramètres élastiques nécessite des mesures très précises, car situées dans le domaine de très petites déformations. Les essais cycliques spéciaux tels que ceux développés par ELHOSRI (1984) ou la colonne résonnante (BOELLE, 1983) permettent d'arriver à cette fin. D'une manière générale, dans les essais drainés, et si la vitesse de déformations est faible les déformations élastiques seront négligeables devant les déformations plastiques pour des déformations totales supérieures à 0,005 . Dans ces conditions, la détermination précise des paramètres élastiques n'est pas pertinente. Dans les essais non drainés au contraire la décomposition théorique de la déformation en partie élastique et plastique, ainsi que l'incompressibilité relative de l'eau imposent la condition suivante:

$$
\partial_{t} \epsilon_{\mathrm{V}}{ }^{\mathrm{e}}=-\partial_{t} \epsilon_{\mathrm{V}}{ }^{p}
$$

On en déduit la variation de pression effective :

$$
\partial_{\mathrm{t}} \mathrm{p}^{\prime}=-\mathrm{K} \partial_{\mathrm{t}} \epsilon_{\mathrm{v}} \mathrm{p}^{\mathrm{s}}
$$

Si les paramètres plastiques ont été calés sur des essais drainés et que l'on manque d'informations sur les paramètres élastiques, il est possible à ce niveau de jouer sur les paramètres élastiques.

L'angle de frottement $\phi$ dans la description de la loi est l'angle de frottement sur le palier de plasticité parfaite à l'état critique. Des essais triaxiaux drainés (avec mesure des variations de volume) ou non drainés (avec mesure des pressions interstitielles) à des contraintes effectives de confinement différentes conviennent. Le module de compressibilité plastique $\beta$ est obtenu par le concept d'état critique. On remarque que, par exemple, le paramètre d'écrouissage déviatoire a joué surtout sur la pente initiale de la surface de charges dans le plan ( $p-q)$ et que le paramètre $\mathrm{b}$ (également $\mathrm{p}_{\mathrm{c}^{\circ}}$ ) joue plutôt sur l'abscisse des pics. D'une manière générale des procédés relativement systématiques (HAJAL, 1984) et des plages de valeur des paramètres suivant les types de matériau ont été élaborés. Plus récemment, un logiciel d'identification automatique des paramètres PARASOL, a été développé, couplé à une base de données.

Afin d'illustrer rapidement les possibilités de la loi de comportement depuis les petites jusqu'aux moyennes déformations, les exemples ci-dessous (fig. 6, 7), tirés de HUJEUX (1985) montrent un fonctionnement convenable de la loi, aussi bien pour les essais monotones que cycliques, drainés ou non drainés et permettent d'apprécier si les principaux aspects mentionnés en début de cette section sont bien pris en compte. Ceux-ci sont évidemment essentiels à une modélisation correcte du problème numérique, qui sera développée dans la section suivante.

$\tau$

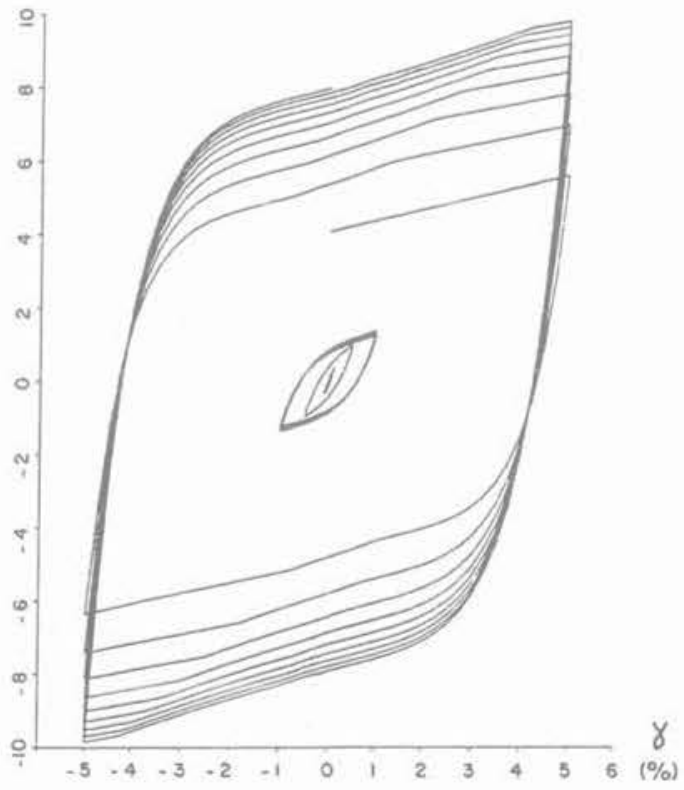

Fig. 4. - Simulation du comportement cyclique depuis les très petites $\left(10^{-5}\right)$ jusqu'aux moyennes déformations $\left(10^{-2}\right)$. Fig. 4. - Cyclic behaviour simulation from very small $\left(10^{-5}\right)$ to small $\left(10^{-21}\right)$ stains. 
COMPARAISON MODELES SEED ET J.C. HUJEUX

G/GMAX en fonction de la distortion

Amortissement en fonction de la distortion

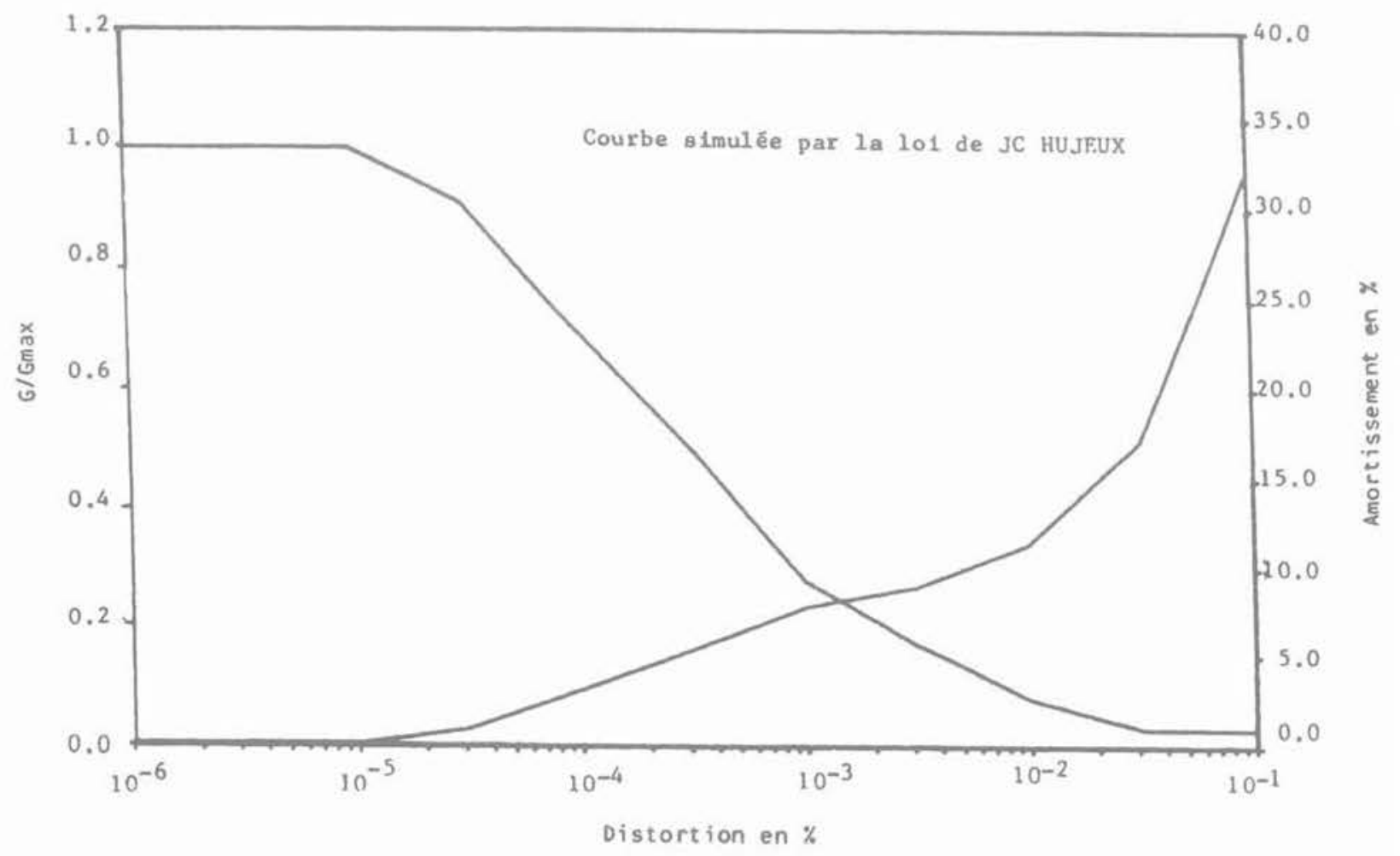

Fig. 5. - Courbes $(G-\gamma)$ et $(\xi-\gamma)$ simulées par la loi des comportements.

Fig. 5. $-(G-\gamma)$ and $(\xi-\gamma)$ curves simulated by the constitutive model.

\section{MODÉLISATION NUMÉRIQUE EN DYNAMIQUE DES SOLS TRANSITOIRE NON LINÉAIRE}

Après avoir établi la formulation variationnelle du problème, les différentes équations sont discrétisées, par rapport à l'espace en éléments finis et au temps par un schéma de Newmark mixte implicite-explicite. La discrétisation totale est alors mise en ceuvre directement du point de vue informatique. Il faut noter que la résolution numérique du système d'équations présentées nécessite des méthodes performantes et rapides. Le choix des algorithmes de résolution et d'intégration est discuté et la stabilité du schéma numérique est présenté dans ce chapitre. Enfin, si la discrétisation d'un milieu continu nous permet de trouver une solution approchée acceptable pour le problème posé, elle est toujours entachée d'effets parasites qui peuvent perturber complètement la solution obtenue, en particulier dans le cas dynamique tels que par exemple des diffractions sur des éléments de tailles différentes, des dispersions numériques ou une propagation anisotrope due au maillage.

Dans ce qui suit, pour alléger la présentation, nous utiliserons les notations suivantes, pour deux champs de vecteurs $\left(v_{1}, v_{2}\right)$, ou de tenseurs $\left(\tau_{1}, \tau_{2}\right)$, définis sur le domaine $\Omega$, ou sur sa frontière $\Gamma$ :

$$
\begin{aligned}
& \left(\tau_{1}, \tau_{2}\right)_{\Omega}=\ln _{\Omega} \tau_{1} \cdot \tau_{2} \mathrm{~d} \Omega=\Sigma_{i j} \ln _{\Omega} \tau_{1 \mathrm{ij}} \cdot \tau_{2 \mathrm{if}} \mathrm{d} \Omega \\
& \left(v_{1}, v_{2}\right)_{\Omega}=l_{\Omega} v_{1} \cdot v_{2} d \Omega=\Sigma_{1} l_{\Omega} v_{11} \cdot v_{21} d \Omega \\
& \left\langle v_{1}, v_{2}\right\rangle_{\Gamma}=\left.\right|_{\Gamma} v_{1} \cdot v_{2} d \Gamma=\left.\Sigma_{i}\right|_{\Gamma} v_{11} \cdot v_{2 i} d \Omega
\end{aligned}
$$




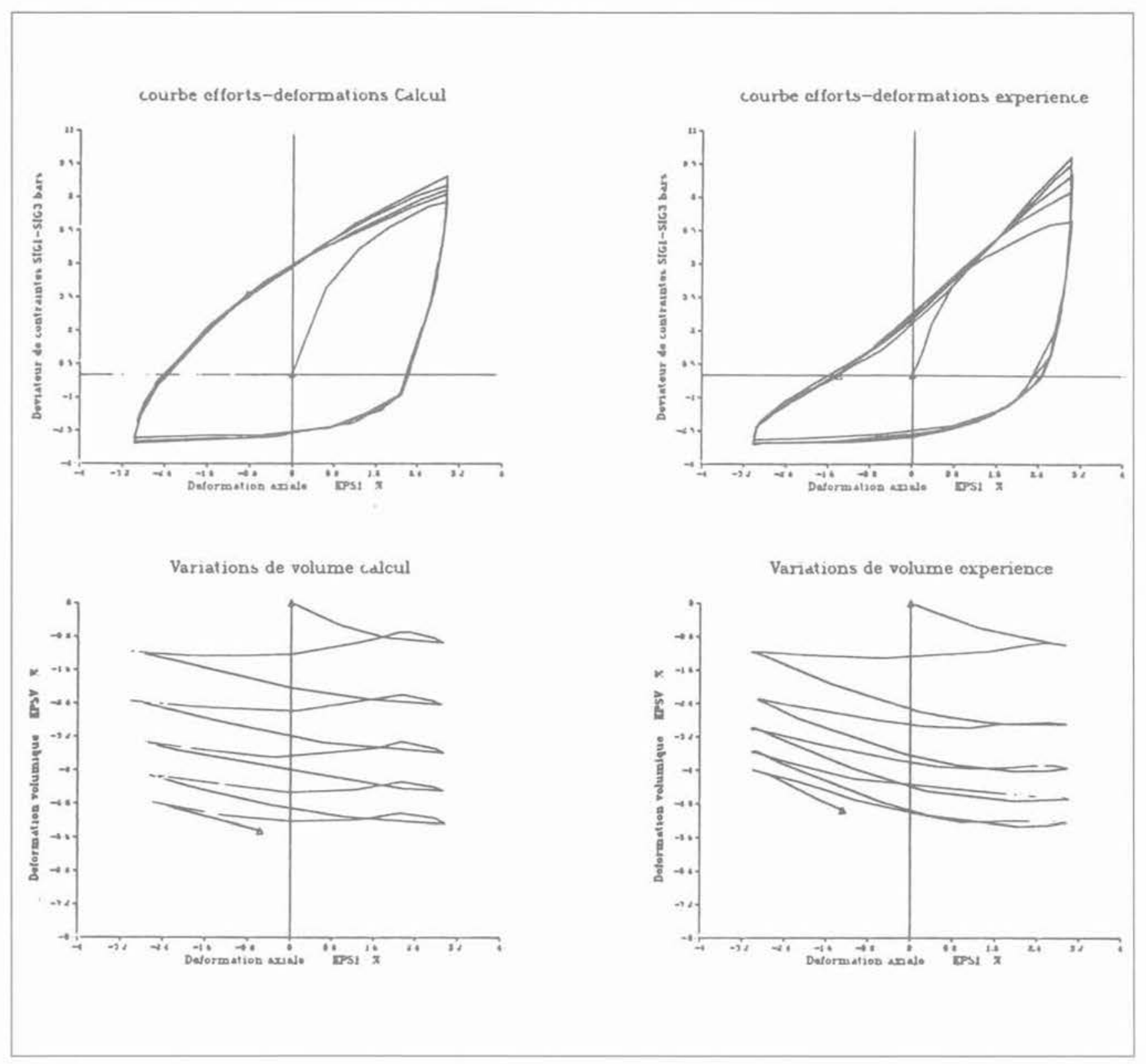

Fig. 6. - Simulation du comportement cyclique drainé du sable d'Hostun. Fig. 6. - Cyclic drained behaviour of Hostun sand.

\subsection{Principe des travaux virtuels ou formulation variationnelle}

En conservant la même définition pour la loi de comportement, on écrit la formulation variationnelle du problème. Soit $V_{3}$ le champ de déplacements virtuels cinématiquement admissibles :

$V_{s}=w_{s} / w_{s}$ régulier sur $\Omega / w_{s}=0$ sur $\Gamma_{u s}$

Soit $\mathrm{Q}$ le champ de pressions virtuelles admissibles, c'està-dire définies par:

$$
\left.\mathrm{Q}=\mathrm{q} / \mathrm{q} \text { régulière sur } \Omega / \mathrm{q}=0 \text { sur } \Gamma_{\mathrm{p}}\right\}
$$

alors la formulation variationnelle du modèle $(u-p)$ s'écrit (MODARESSI, 1987) :

$$
\begin{aligned}
& \left(\rho \partial_{n} u_{s}, w_{s}\right)_{\Omega}+\left(\sigma^{\prime \prime}, \epsilon\left(w_{s}\right)\right)_{\Omega}-\left(\alpha p, \operatorname{div} w_{s}\right)_{n} \\
& =\left(\rho \mathrm{g}, \mathrm{w}_{5}\right)_{\Omega}+\left\langle\mathrm{T}, \mathrm{w}_{5}\right\rangle_{\mathrm{ro}} \\
& -\left(\rho_{1} \mathrm{~K} \operatorname{div} \partial_{\mathrm{u}} \mathrm{u}_{\mathrm{s}}, \mathrm{q}\right)_{0}-\left(\alpha \operatorname{div} \partial_{\mathrm{t}} \mathrm{u}_{\mathrm{s}}, q\right)_{\Omega} \\
& -(\partial, p / Q, q)_{\Omega}-(K \operatorname{grad} p \cdot \operatorname{grad} q)_{\Omega} \\
& =\langle\varphi, q\rangle_{\Gamma_{\varphi}}-\left(\mathrm{K} \operatorname{grad}\left(\rho_{1} \mathrm{~g} X\right) \text {, grad } q\right)_{\Omega}
\end{aligned}
$$

Le problème initial peut se formuler de la manière suivante :

Problème variationnel: chercher $u_{s}(t) \in V_{s}, p(t) \in Q$ et $(\sigma$ " $(t), \alpha(t))$ vérifiant la formulation variationnelle, la loi de comportement et les conditions initiales. 


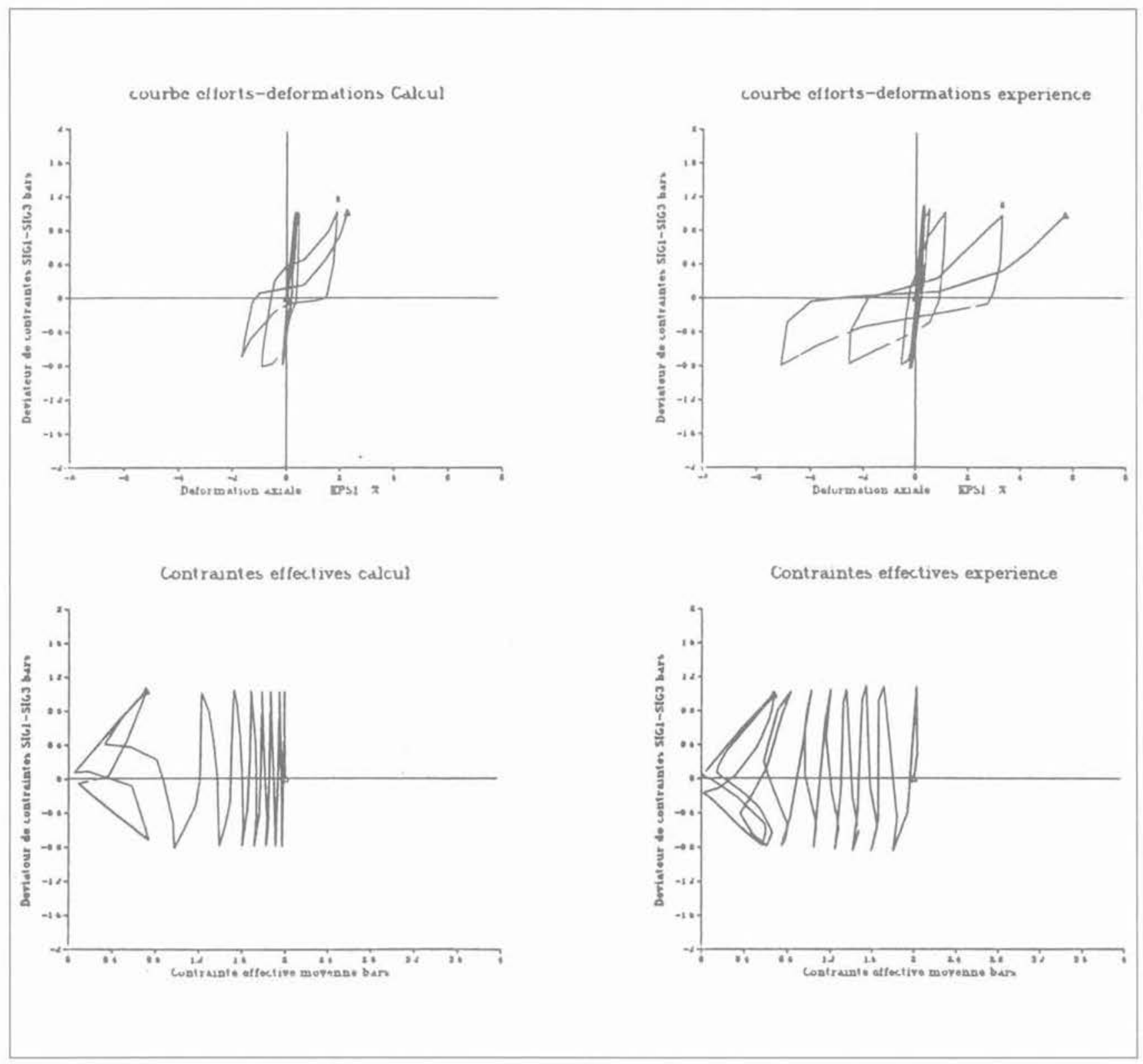

Fig. 7. - Simulation du comportement cyclique non drainé du sable d'Hostun.

Fig. 7 . - Cyclic undrained behaviour of Hostun sand.

On vérifie, comme cela avait été mentionné dans le choix retenu des conditions aux limites, que cette formulation est bien adaptée aux conditions aux limites en contraintes totales sur $\Gamma_{\sigma}$ et en flux sur $\Gamma_{\varphi}$. Cela servira ultérieurement, pour raccorder des zones mono et biphasiques.

\subsection{Approximation en espace par éléments finis}

Une formulation isoparamétrique classique est employée pour discrétiser le problème ci-dessus par la méthode des éléments finis. Les champs de déplacements $u_{s}(t)$ et de pression $\mathrm{p}(\mathrm{t})$, définis dans les espaces de dimensions infi- nies, seront approchés par des champs $u_{s h}(t)$ et $p_{h}(t)$ définis dans les espaces $V_{\text {sh }}$ et $Q_{h}$ de dimensions finies, construits à partir des fonctions de base générées par la méthode des éléments finis. Pour chercher les approximations des autres inconnues $\sigma$ " $(\mathrm{t})$ et $\alpha(\mathrm{t})$ il faut approcher ces inconnues par des fonctions constantes sur chaque sous-élément défini par l'intégration numérique choisie et égales à leurs valeurs aux points d'intégration.

La formulation variationnelle approchée s'écrit alors :

Problème variationnel approché : chercher $u_{\text {sh }}(t) \in V_{\text {sh }}$, $p_{h}(t) \in Q_{h}$ et $\left(\sigma{ }^{\prime \prime}(t), \alpha_{h}(t)\right)$ vérifiant les équations précédentes et les conditions initiales; $\forall w_{\text {sh }} \in V_{\text {sh }}, \forall q_{h}$ $\epsilon Q_{h}$. 
Remarque : La discrétisation en espace étant classique, nous renvoyons aux ouvrages sur la méthode des éléments finis où les fonctions de base relatives à ces éléments sont définies.

\section{Mise en cuvre numérique}

A chaque nœud du maillage, on associe une fonction de base $w_{3}$ polynomiale sur chaque élément, égale au nceud J, nulle sur les autres. En introduisant des sommations sur les directions de l'espace et sur les noeuds libres, on pose:

$u_{\mathrm{sh}}=\Sigma u_{\mathrm{sli}} \cdot \mathrm{w}_{\mathrm{sl}} e_{\mathrm{i}}$

$\mathrm{p}_{\mathrm{h}}=\Sigma \mathrm{p}_{1} \cdot \mathrm{w}_{\mathrm{fl}}$

$w_{\mathrm{sh}}=w_{\mathrm{se}_{2}} \cdot e_{\mathrm{f}}$

$\mathrm{q}_{\mathrm{h}}=\mathrm{w}_{\mathrm{i}, \mathrm{s}}$

Pour simplifier la présentation, les mêmes fonctions de base pour le déplacement du squelette solide et la pression interstitielle dans le fluide sont utilisées. Cependant il semble intéressant de prendre pour la pression des fonctions de base d'ordre inférieur par rapport au déplacement du squelette solide, en particulier lorsque l'eau est considérée comme étant très faiblement compressible. L'introduction des développements précédents dans les équations variationnelles approchées donnent finalement :

$$
\begin{aligned}
& \Sigma \partial_{n} u_{s i l} \delta_{i j}\left(\rho w_{1}, w_{j}\right)+\left(\sigma^{\prime \prime}, \epsilon\left(w_{j} \cdot e_{j}\right)\right)_{n} \\
& -\alpha \Sigma \mathrm{p}_{1}\left(w_{1}, \operatorname{div} w_{\mathrm{J}} e_{j}\right)=\left(\rho \mathrm{g}, w_{\mathrm{j}} \cdot e_{\mathrm{j}}\right)_{0}+\left\langle\mathrm{T}, \mathrm{w}_{\mathrm{j}} \cdot e_{\mathrm{j}}\right\rangle_{\mathrm{ra}} \\
& -\Sigma \partial_{t t} u_{s h}\left(\operatorname{div} \rho_{4} K w_{1} e_{1}, w_{j}\right)-\alpha \Sigma \partial_{t} u_{s h}\left(\operatorname{div} w_{1} e_{1}, w_{j}\right) \\
& -\Sigma \partial_{1} p_{1}\left(1 / Q w_{1}, w_{j}\right)-\Sigma p_{1}\left(K, \operatorname{grad} w_{1}, \operatorname{grad} w_{j}\right) \\
& =\left\langle\varphi, w_{\jmath}\right\rangle_{\Gamma_{\varphi}}-\left(\mathrm{K} \operatorname{grad}\left(\rho_{1} \mathrm{~g} X\right), \operatorname{grad} w_{\jmath}\right)_{\mathrm{Q}}
\end{aligned}
$$

Le caractère couplé des équations mécanique et hydraulique apparaît à l'évidence dans le système différentiel en temps, ci-dessus, puisque les déplacements du squelette, ainsi que les pressions interstitielles figurent simultanément dans les deux équations.

\subsection{Discrétisation en temps}

La semi-discrétisation en espace a permis de transformer les équations aux dérivées partielles en système d'équations différentielles ordinaires. L'évolution des inconnues dans le temps est donnée grâce à la discrétisation en temps. Ici nous utilisons la méthode d'intégration pas à pas et en particulier un schéma mixte Implicite-Explicite qui a été proposé par HUGHES et LIU (1978). L'avantage de ce genre de technique mixte est de pouvoir traiter des zones où les vitesses de propagation peuvent être très différentes, en tirant avantage des aspects complémentaires d'un schéma implicite et explicite. L'algorithme Implicite-Explicite est composé de l'algorithme implicite de Newmark et un algorithme explicite prédicteur-correcteur. Le domaine $\Omega$ est partitionné en deux sous-domaines $\Omega_{1}$ et $\Omega_{\mathrm{E}}$ correspondant aux zones implicite et explicite.

Soit alors 10. T[ l'intervalle en temps de l'analyse. On découpe cet intervalle en pas de temps $\Delta t$ non nécessairement égaux. Cependant, pour une plus grande sim- plicité des équations, nous supposerons ces pas de temps égaux, si bien qu'à l'étape $\mathrm{n}$, on essaye d'approcher $u_{s h}(n \Delta t), \sigma_{\text {sh }}^{\prime \prime}(n \Delta t), p_{h}(n \Delta t) \ldots$, par une suite : $\mathrm{u}_{\text {shn }}, \sigma_{\text {shn. }}, \mathrm{p}_{\mathrm{hn}}$. Cornme, dorénavant, dans cette étude, il ne sera plus question que de la discrétisation en temps des approximations par éléments finis de $u_{s h}(t), \sigma_{\text {sh }}(t)$. $\left.u_{r h}(t)\right), p_{h}(t) \ldots$, nous supprimerons dans la suite l'indice h.

Cette notation étant retenue, lalgorithme en temps se définit à partir d'un algorithme standard de Newmark. muni des paramètres classiques $(\beta, \gamma)$, mais en introduisant, en outre les notions de prédicteur et de correcteur suivants : soit $\left(u_{s n}, \sigma_{s n}^{\prime}, v_{s n}, a_{s n}\right)$, une suite censée approcher les déplacements, contraintes, vitesses et accélérations de la partie solide, et soit une suite semblable pour la partie pression interstitielle. On définit alors, dans le cadre de la méthode mixte:

\section{Le prédicteur de Newmark:}

$$
\begin{aligned}
& u_{n+1}=u_{n}+\Delta t v_{n}+\Delta t^{2}(1-\beta / 2) a_{n} \\
& v_{n+1 *}=v_{n}+\Delta t(1-\gamma) a_{n} \\
& \sigma^{\prime \prime}{ }_{n+1^{*}}=\sigma_{s n}^{\prime \prime}+\Delta t D^{e p} ; \epsilon\left(u_{n+1^{*}}-u_{n}\right) .
\end{aligned}
$$

Le correcteur de Newmark:

$$
\begin{aligned}
& u_{n+1}=u_{n+1}+\Delta t^{2} \beta a_{n+1} \\
& v_{n+1}=v_{n+1}+\Delta t \gamma a_{n+1} \\
& \sigma_{s n+1}^{\prime \prime}=\sigma^{\prime \prime}{ }_{s n}+\Delta t D_{e p} ; \epsilon\left(u_{n+1}-u_{n}\right)
\end{aligned}
$$

des expressions similaires étant déduites pour les pressions interstitielles.

En injectant ces expressions dans les équations obtenues précédemment, on aboutit à la résolution d'un système non linéaire en $u_{n+1}$ pour la partie implicite et linéaire trivial en $u_{n+1}$. pour la partie explicite. Les itérations ne porteront au cours de l'étape $(n+1)$ que sur les premiers termes.

\section{Remarque:}

Dans le cadre de la loj élastoplastique retenue, la méthode d'intégration de la loi de comportement doit être très précise, vu les très fortes non linéarités. Néanmoins, lă encore, la forme envisagée ici permet d'exposer convenablement l'algorithme implicite-explicite, sans lourdeur excessive de notations. L'intégration précise de la loi de comportement est discutée plus loin.

\section{Remarque :}

Dans cette approche, ce sont les éléments finis qui sont déclarés implicites ou explicites, et non leurs nœeuds, ce qui fait qu'il n'y a pas des déplacements de nouds qui sont "en avance * ou "en retard " par rapport à d'autres ; ce sont seulement des contributions énergétiques qui sont prédites et corrigées. La conséquence essentielle est que l'interface entre les deux zones est traitée sans ambiguĩté. 


\subsection{Frontières absorbantes et conditions aux limites à l'infini}

Comme cela a été rappelé dans l'introduction, très souvent en géotechnique et en génie parasismique, le domaine de sol à étudier au voisinage des hétérogénéités locales est de très grande dimension, par rapport à celles-ci, et doit être modélisé comme un milieu infini. Cela est la source de difficultés numériques pour une méthode telle que la méthode des éléments finis car des réflexions d'ondes parasites se produisent à la frontière du maillage. Une technique de frontière absorbante, basée sur lapproximation paraxiale est présentée dans ce paragraphe, ainsi que la mise en œuvre d'un élément de frontière qui permet d'utiliser la méthode des éléments finis même dans le cas d'un domaine non borné, Cependant, il faut noter que si du point de vue de cont de calcul cette méthode n'est pas comparable avec les méthodes semi-analytiques comme la méthode d'AkiLarner, c'est la seule méthode qui permette de travailler directement dans le domaine temporel, pour des ondes incidentes sur le maillage non nécessairement normales.

\subsection{Partition du domaine infini de sol}

Pour étudier le problème, lorsqu'on est en présence d'un sol élastoplastique, nous partageons le domaine infini en lastiques et modélisé par les équations des précédents chapitres. Ces sédiments sont situés à proximité de la surface libre. Ce domaine est borné, mais de géométrie quelconque :

- le domaine $\Omega_{\text {sm }}$, constitué de sédiments non élastiques et monophasiques. Ce domaine réalise une zone de transition éventuelle entre $\Omega_{\mathrm{d}}$ et $\Omega_{c}$, et est borné.

Le comportement du sol dans le domaine $\Omega_{\mathrm{s}}$ est élastique, monophasique. Ce domaine est non borné. Le comportement de ce domaine sera modélisé par l'approximation paraxiale des équations de l'élastodynamique. La stratigraphie dans $\Omega_{s^{\prime}}$ est suffisamment simple, de telle manière que le champ sismique incident se propageant dans celle-ci, puisse être calculé soit analytiquement, soit par une méthode du type ThomsonHaskell. Pour fixer les idées, on pourra se contenter du cas d'un matériau homogène.

L'ensemble des équations traduisant la modélisation s'écrit de la manière suivante.

\section{Dans $\Omega_{s^{\prime}}$}

Le champ de déplacement $\mathrm{u}_{s}$ vérifie les équations de l'élastodynamique transitoire, c'est-à-dire en supposant, en outre que le matériau est homogène isotrope. A l'infini. le champ $u$, doit tendre vers le champ sismique incident $u_{i}$ qui, pour fixer les idées, peut être pris sous

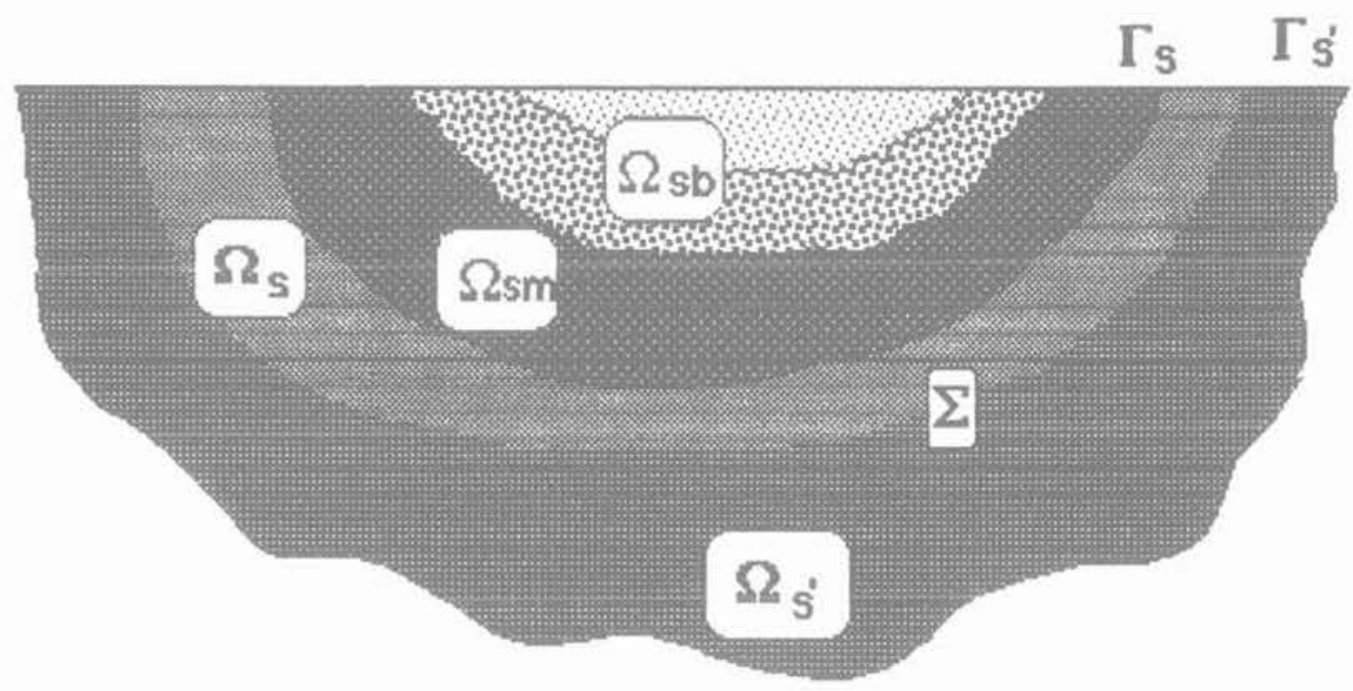

Fig. 8. - Partition du domaine du sol.

Fig. 8. - Soil domain decomposition.

deux parties $\Omega_{\text {s }}$ et $\Omega_{\text {s }}$ (fig. 8 ). D'une manière générale, les hétérogénéités quelconques sont dans le domaine $\Omega_{s}$, alors que le domaine extérieur $\Omega_{s}$, est relativement régulier et homogène. Les hypothèses suivantes sont retenues.

Le domaine $\Omega$, lui-même est décomposé en deux parties :

- le domaine $\Omega_{\mathrm{sb}}$ constitué de sédiments poreux, ané- forme d'ondes planes, compatibles avec la stratigraphie horizontale de $\Omega_{s}$. On a donc:

$$
\lim u_{s}=u_{i} \text { lorsque }|x| \rightarrow \infty
$$

Le tenseur des contraintes dans $\Omega$. s'écrit comme la somme des contraintes statiques $\sigma_{5^{\circ}}{ }^{\circ}$ (présismique) et des contraintes dynamiques.

$$
\sigma_{s^{\prime}}=\sigma_{s^{0}}{ }^{0}+\sigma_{s^{\prime}}\left(\mathrm{u}_{s^{\prime}}\right)
$$




\section{Interface $\Sigma$ entre $\Omega_{s}$ et $\Omega_{s^{\prime}}$}

A l'interface $\Sigma$ entre $\Omega_{\text {s }}$ et $\Omega_{\text {s }}$ on doit écrire la continuité des déplacements et des vecteurs contraintes:

$\mathrm{u}_{\mathrm{s}}=\mathrm{u}_{\mathrm{s}}$

$t_{s}+t_{s}\left(u_{s^{\prime}}\right)=0$.

Rappelons que le comportement est supposé élastique dans un voisinage de $\Sigma$, si bien que la notation $t_{s^{\prime}}\left(u_{s}\right)$, a bien un sens :

$t_{s^{\prime}}\left(u_{s^{\prime}}\right)=\sigma_{s^{\prime}}\left(u_{s^{\prime}}\right) \cdot n_{s^{\prime}}=\lambda\left(\operatorname{div} u_{s^{\prime}}\right) n_{s^{\prime}}+2 \mu \epsilon\left(u_{s^{\prime}}\right) \cdot n_{s^{\prime}}$

Dans $\Omega_{s m}$

Le comportement élastoplastique des sédiments est régi par les équations classiques de Pélastoplasticité dynamique. Le champ de contraintes dans $\Omega$, doit satisfaire des conditions de surface libre sur $\Gamma_{s}$.

\section{Dans $\Omega_{s b}$}

Le comportement des sédiments dans ce domaine est décrit par le modèle biphasique présenté plus haut. Nous ne réécrivons pas ces équations, cependant, il est important de décrire les conditions de raccord entre $\Omega_{\mathrm{sm}}$ et $\Omega_{\mathrm{sb}}$.

\section{Raccord entre $\Omega_{s b}(u-p)$ et $\Omega_{s m}$}

A l'interface, on doit écrire la continuité du déplacement de la phase solide, la continuité du vecteur contrainte totale, et la nullité de la vitesse d'écoulement :

$u_{s b}=u_{s m}, K \cdot$ grad $p \cdot n_{s b}=0$

$t_{s b}+t_{s m}=0$.

Les mêmes remarques que précédemment s'appliquent dans le cas du raccord avec un milieu poreux quasiment imperméable, ou au contraire quasiment perméable. On notera ici une nouvelle fois que les conditions aux limites retenues au départ et la formulation variationnelle qui en découle sont également bien adaptées aux conditions de raccord entre une zone biphasique et une zone monophasique. Dans la pratique, il s'avère que la possibilité de mixer de telles zones est particulièrement intéressante.

\section{Conditions initiales}

On a donc maintenant écrit toutes les équations à notre disposition à chaque instant $\mathrm{t}$ successivement dans le domaine extérieur élastique $\Omega_{s}$ et dans le dornaine intérieur élastoplastique $\Omega_{s}$. II faut en outre adjoindre des conditions initiales qui sont du type de celles qui avaient été développées dans la section précédente.

\subsection{Approximation paraxiale}

Nous présentons ici les principales étapes de la construction de frontières absorbantes, basées sur l'approximation paraxiale des ondes diffractées vers l'infini. Pour les détails nous renvoyons à COHEN (1984) et MODARESSI (1987).

\section{a. Impédance spectrale de l'interface $\Sigma$}

Pour construire systématiquement lapproximation paraxiale, on procède à une transformée de Fourier des équations de l'élastodynamique par rapport au temps, ainsi qu'aux deux variables d'espace dans le plan tangent à la frontière $\Sigma$. lci nous nous limiterons au cas où $\Sigma$ est une surface plane. D'autre part, nous n'exa. minerons pas non plus le cas de frontière avec coin pour lequel nous renvoyons à ENGQUIST et MAJDA (1979).

Pour évaluer l'impédance de la frontière $\Sigma$, il faut calculer le vecteur contrainte $t_{0}$ sur la facette de normale $e_{3}$ en $x_{3}=0$. On peut faire subir à $t\left(x^{\prime}, x_{3}\right)$ la même transformée de Fourier, si bien que dans le domaine de Fourier, on obtient l'impédance spectrale de la frontière $\Sigma$ sous la forme:

$$
\hat{t}_{0}=A\left(\left|\xi^{\prime}\right|, \omega\right) \hat{u}_{0}^{\prime}\left(\xi^{\prime}, \omega\right)
$$

où $\mathrm{A}$ désigne l'opérateur impédance spectrale globale, $\omega$ la pulsation, $\xi$ ' le vecteur d'ondes. Revenant à l'espace physique, par la transformée de Fourier inverse, on obtient :

$t_{0}\left(x^{\prime}, t\right)=$

$\left.(2 \pi)^{-3 / 2}|A| \xi^{\prime} \mid, \omega\right) \hat{u}_{0}\left(\xi^{\prime}, \omega\right) \exp \left[1\left(\xi^{\prime} x^{\prime}+\omega t\right)\right] d \xi^{\prime} d \omega$

\section{b. Approximation paraxiale de limpédance}

La relation précédente représente l'action spectrale exercée par le domaine extérieur $\Omega_{s^{\prime}}$ sur le domaine intérieur $\Omega_{s}$ lorsque $\Omega_{s^{\prime}}$ est le siège d'ondes rayonnant à partir de $\Sigma$ vers l'extérieur. Cette impédance n'est pas locale puisqu'elle fait intervenir $\hat{a}_{0}^{\prime}(\xi, \omega)$ transformée de Fourier de $\hat{u}_{0}\left(x^{\prime}, t\right)$ pour tout $x^{\prime}$ et $t$. L'idée est alors de développer les nombres d'ondes relatifs aux ondes $\mathrm{P}$ et $\mathrm{S}, \xi_{s}$ et $\xi_{\mathrm{p}}$ selon les puissances de $\left|\xi^{\prime}\right| / \omega$ (ENGQUIST et MAJDA, 1977). Cette approximation sera bonne soit à haute fréquence, soit pour $\left|\xi^{\prime}\right|$ petit. On voit ainsi que, pour $|\xi '|$ petit, on aura des ondes se propageant selon des directions voisines de $e_{3}$, comme cela est indiqué sur la figure 9.

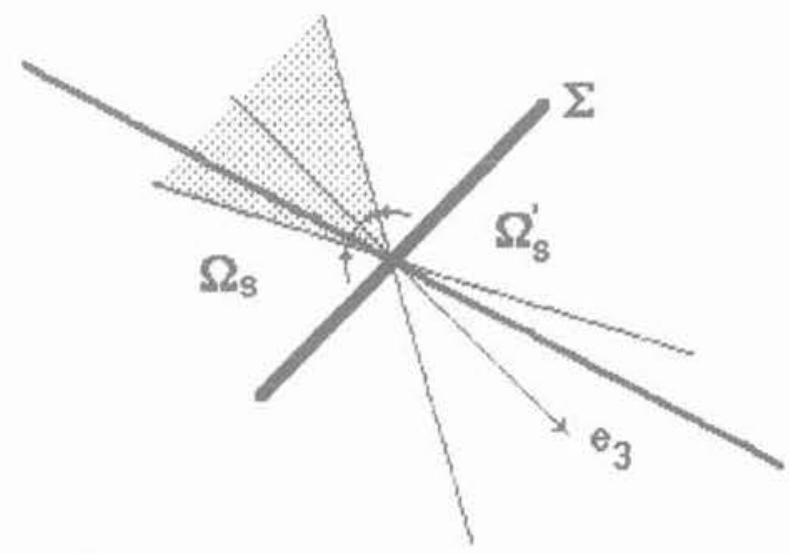

Fig. 9. - Frontière absorbante et interface entre le domaine extérieur et intérieur.

Fig. 9. - Absording boundary and the interface between outer and inner domains.

Si on utilise des développements limites pour $\xi_{p}$ et $\xi_{s}$, en multipliant par une puissance convenable de $\omega$, de 
manière à supprimer cette quantité au dénominateur, on obtiendra :

$$
A_{0}\left(\xi^{\prime}, \omega\right) t_{0}=A_{1}\left(\xi^{\prime}, \omega\right) \hat{u}_{0}
$$

où $A$ est une fonction polynômiale de $\xi$ et de $\omega$. On pourra se reporter à ENGQUIST et MAJDA (1979) pour un calcul détaillé des $A_{{ }_{*}}$ Par exemple à l'ordre zéro on obtient :

$$
t_{s}=\rho c_{p} \partial_{1} u_{3} e_{3}+\rho c_{s} \partial_{1} u_{s}
$$

qui est précisément l'impédance transitoire d'amortisseurs distribués le long de la frontière $\Sigma$ très souvent utilisés en pratique. A l'ordre un, on obtient une expression plus compliquée, où on voit apparaître la dérivée par rapport au temps du vecteur contrainte.

La conclusion essentielle de cette section est que lapproximation paraxiale dans le domaine extérieur $\Omega_{s}$ permet d'obtenir une impédance locale transitoire ne faisant intervenir que les dérivées intérieures, à la frontière $\Sigma$ de raccordement. On retiendra sous forme symbolique les expressions suivantes à l’ordre zéro et un, respectivement :

$t_{s}=A_{0}\left(\partial_{t} u_{s}\right)$

$\partial_{t_{s}}=A_{1}\left(\partial_{n} u_{s} \partial_{i} u_{s} u_{s}\right)$

pour le champ rayonnant vers l'extérieur de $\Omega_{\mathrm{s}}$.

c. Formulation variationnelle du raccord sur $\Sigma$ On rappelle que le voisinage de l'interface $\Sigma$ appartenant à $\Omega_{s}$ a un comportement supposé élastique linéaire. A l'infini le champ total $u_{s}$ doit être égal au champ sismique incident $\mathrm{u}^{\prime}$ généré par le mécanisme au foyer: on a donc :

$\left(u_{s}-u_{i}^{\prime}\right)$ vérifie les conditions de radiation à l'infini.

En pratique on décompose le champ total $u_{\text {s }}^{\prime}$ en champ incident et champ diffraction-rayonnement u', sur l'interface $\Sigma$ :

$$
u_{s}^{\prime}=u_{1}^{\prime}+u_{\text {, }}^{\prime}
$$

Sur l'interface $\Sigma$ on aura pour le champ rayonnant dans $\Omega_{s}^{\prime}$ due à la diffraction sur $\Sigma$, et pour lapproximation d'ordre zéro:

$$
t_{s}\left(u_{x}^{\prime}\right)=A_{0}\left(\partial_{i} u_{x}^{\prime}\right)
$$

Ce champ rayonné obéissant à l'équation paraxiale vérifie donc la condition de radiation approximativement. La continuité du vecteur contrainte sur l'interface $\Sigma$ et l'hypothèse de linéarité au voisinage de $\Sigma$ permettent d'écrire, dans le cas de l'approximation paraxiale d'ordre zéro :

$$
\begin{aligned}
t_{s} & =-t_{s}\left(u_{s}^{\prime}\right)=-t_{s}\left(u_{j}^{\prime}\right)-t_{s}\left(u_{t}^{\prime}\right) \\
& =-t_{s}\left(u_{1}^{\prime}\right)-A_{0}\left(\partial_{1} u_{t}^{\prime}\right) \\
& =-t_{s}\left(u_{s}^{\prime}\right)-A_{0}\left(\partial_{1} u_{s}\right)+A_{0}\left(\partial_{1} u_{\prime}^{\prime}\right)
\end{aligned}
$$

qui donne explicitement la sollicitation due au champ sismique libre, c'est-à-dire sans la présence des hétérogénéités locales que l'on souhaite étudier.

Dans le domaine $\Omega_{\mathrm{sb}}$ contenant le matériau poreux biphasique, la formulation variationnelle est celle présentée précédemment. Les conditions aux limites envisagées alors, permettent de traiter directement les conditions de raccord entre une telle zone et la zone de matériau monophasique, que nous avons mentionnées au début de ce chapitre. Par conséquent, nous nous limitons maintenant à la formulation variationnelle permettant de raccorder les zones $\Omega_{\text {sm }}$ correspondant à un domaine borné constitué de matériau monophasique (éventuellement anélastique) au domaine $\Omega_{c}$ non borné, constitué d'un matériau élastique et dont la propagation des ondes est approchée par l'équation paraxiale.

Soit alors, w un champ de déplacements virtuels cinématiquement admissibles dans $\Omega_{\text {sin }}$, on écrit à partir du principe des travaux virtuels pour tout $w$ :

$\left(\rho \partial_{\mathrm{u}} \mathrm{u}_{s}, w\right)_{\Omega_{s m}}+\left(\sigma_{s}, \epsilon(w)\right)_{\Omega_{\mathrm{sm}}}-\left\langle\mathrm{t}_{\mathrm{s}}, w\right\rangle_{\Sigma}=(\rho \mathrm{g}, w)_{\Omega_{s m}}$ La loi de comportement est celle que nous avons présentée plus haut. $\mathrm{Si}$ on prend en compte l'expression obtenue plus haut du vecteur contrainte à l'interface, on en déduit

$$
\begin{gathered}
\left(\rho \partial_{t t} u_{s}, w\right)_{\Omega_{s m}}+\left(\sigma_{s}, \epsilon(w)\right)_{\Omega_{s m}}+\left\langle A_{0}\left(\partial_{1} u_{s}\right), w\right\rangle_{\Sigma} \\
=(\rho g, w)_{\Omega_{s m}}+\left\langle-t_{s}\left(u_{p}^{\prime}\right)+A_{0}\left(\partial_{1} u_{1}^{\prime}\right), w\right\rangle_{\Sigma}
\end{gathered}
$$

$\mathrm{Ce}$ qui constitue la forme finale des termes à mettre en œuvre dans les calculs. Dans le cas de l'équation d'ordre supérieur, on obtient des équations qui font intervenir la vitesse du vecteur contrainte, qui sera discrétisée ensuite en temps. Nous rappelons simplement que nous utilisons le schéma mixte de Newmark que nous avons présenté en détail dans le chapitre précédent.

\section{APPLICATIONS AU GÉNIE PARASISMIQUE}

Après avoir donné, dans les sections précédentes, les éléments d'une modélisation mécanique et numérique des sédiments saturés, soumis à des sollicitations dynamiques, nous proposons maintenant de montrer comment les différents aspects interviennent d'une manière très couplée dans l'application à deux problèmes aux limites. Tout d'abord, une colonne de sol sera soumise à des ondes $\mathrm{SV}$ ou $\mathrm{P}$ à propagation verticale; cet exemple simple permettra d'appréhender clairement le rôle respectif de la loi de comportement et des variations de volume, ainsi que linfluence des pressions interstitielles. Le deuxième exemple, plus complexe, traitera de l'influence du rôle de l'eau et du comportement non élastique des sédiments sur les effets locaux de site en génie parasismique.

\section{Champ incident}

Le champ incident sous la forme d'onde plane peut contenir différents types de signaux. Pour les tests, nous avons choisi le signal de Ricker utilisé très souvent dans la littérature (RICKER, 1960).

Ce signal est défini de la façon suivante dans le domaine transitoire :

$f(t)=0,5 \pi^{1 / 2}(a-0,5) \exp (-a)$ avec $a=[\pi(t$

où $t_{s}$ est le temps d'amplitude maximale et $t_{p}$ est la période caractéristique. Dans le domaine de Fourier, ce signal s'écrit :

$F(\omega)=\frac{-10^{2} t_{p}{ }^{3} \sqrt{2 \pi}}{16 \pi^{3}} e \frac{-\omega^{2} t_{p}^{2}}{4 \pi^{2}} e^{-\mid \omega t_{s}}$ 

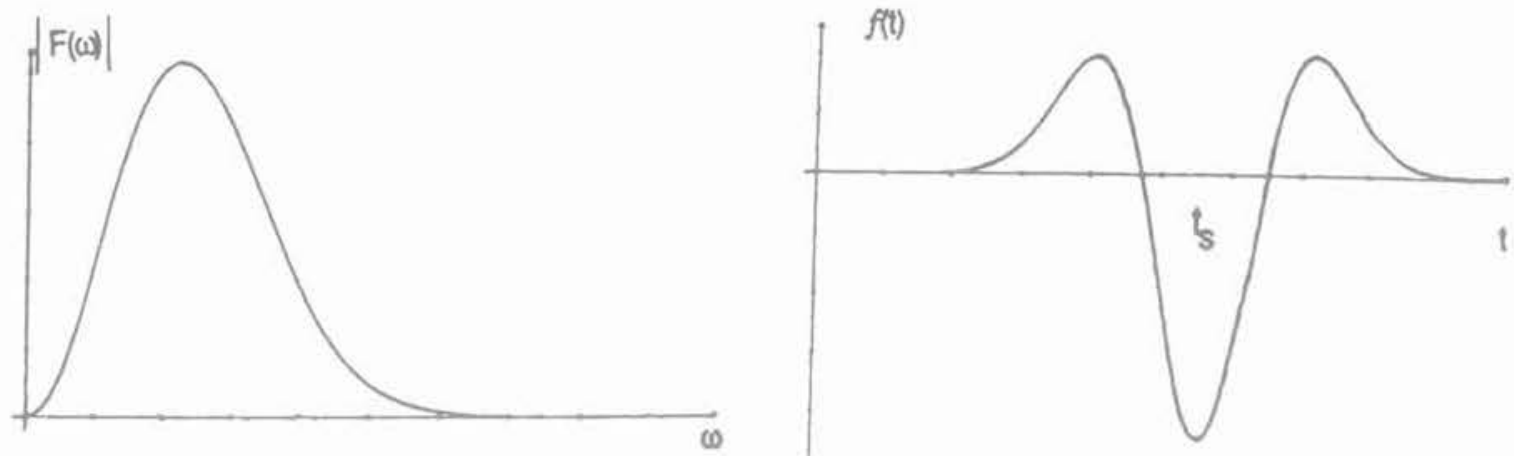

Fig. 10. - Signal de Ricker dans les domaines de fréquence et de temps.

Fig. 10. - Ricker wavelet in time and frequency domains.

la fréquence de pic $\left(\omega_{0}\right)$ est égale à $2 \pi / t_{0}$. Ce signal a l'intérêt d'être simple aussi bien dans le domaine transitoire que dans le domaine de fréquence (fig. 10) et surtout la chute rapide de $F(\omega)$ pour $\omega>\omega_{0}$ ne crée pas d'oscillations de haute fréquence dues à la fréquence de coupure (BARD et BOUCHON, 1980)

Dans le cas réel on peut utiliser les accélérogrammes enregistrés pendant les séismes ou les diagrammes synthétiques obtenus par un calcul préalable sans aucune difficulté. Il convient de noter que dans les résultats présentés plus loin, c'est toujours le déplacement du champ libre qui est imposé, et non l'accélération. Lorsque la fréquence caractéristique du signal de Ricker varie, pour une même amplitude de déplacement imposé, laccélération du champ libre sera nécessairement différente.

\subsection{Colonne de sable lâche (ou moyen ou dense) soumise à une onde $\mathrm{P}$ et $\mathrm{SV}$}

Nous considérons un problème monodimensionnel, représentant une colonne de sol de $10 \mathrm{~m}$ de hauteur. sollicitée par une onde P ou SV verticalement incidente en forme d'un signal de Ricker. Afin d'étudier l'influence du comportement anélastique des sols sur la réponse enregistrée sur la surface libre nous considérons successivement un sable lache, moven et dense dans les 5 premiers mètres de la surface. Le sable choisi est le sable d'Hostun pour lequel les paramètres de la loi de comportement élastoplastique de Hujeux ont été déterminés par ailleurs (HUJEUX, 1985). Les caractéristiques du champ incident sont données dans le tableau 1.

Tableau 1 - Caractéristiques du signal de Ricker. Table 1 - Ricker wavelet characteristics.

\begin{tabular}{|c|c|c|c|c|}
\hline Fréquences & & 2,5 & 5 & $\mathrm{~Hz}$ \\
\hline $\begin{array}{l}\text { Période caractéristique } \\
\text { Temps } \\
\text { de I'amplitude maximale } \\
\text { Amplitude du signal } \\
\text { Pas de temps de calcul }\end{array}$ & $\begin{array}{l}t_{\rho} \\
t_{s} \\
\Delta t\end{array}$ & $\begin{array}{l}0,04 \\
0,04 \\
0,0005 \\
0,001\end{array}$ & $\begin{array}{l}0,2 \\
0,20 \\
0,0005 \\
0,001\end{array}$ & $\begin{array}{l}\sec \\
\text { sec } \\
\text { m } \\
\text { sec }\end{array}$ \\
\hline
\end{tabular}

Pour chaque densité, les profils des déplacements, des vitesses et des accélérations, à la surface, sont présentés pour les ondes incidentes $\mathrm{P}$ et $\mathrm{SV}$ sur les figures 11 et 12 à moyenne fréquence $(25 \mathrm{~Hz})$ et sur les figures 13 et 14 à basse fréquence $(5 \mathrm{~Hz})$. Le cas élastique sert de référence.

\section{a. Cas monophasique}

On peut constater que les déplacements sont toujours amplifiés indépendamment du type ou de la période de l'onde incidente, lorsqu'on est en présence de matériaux anélastiques. Cette amplification est nettement plus importante pour les fréquences élevées lorsque le champ incident est sous la forme d'une onde P. Quant aux ondes SV, ce sont les basses fréquences qui créent l'amplification la plus importante. Dans tous les cas le phénomène est presque toujours accompagné par des déformations irréversibles correspondant à des déplacements horizontaux dans le cas de l'onde SV, et à un tassement dans le cas de l'onde P.

On constate également que les sables moyen et ciense se comportent sensiblement de la même façon et le sable lâche se distingue en montrant très souvent des valeurs d'amplification relativement plus élevées surtout à basses fréquences. Lorsque la fréquence est élevée. le comportement anélastique a pour effet de diminuer l'intensité de l'accélération pour les ondes SV. MOHAMMADIOUN et PECKER (1984) expliquent ce phénomène par le fait que la plastification du matériau plus importante en moyenne sur des chemins déviatoires (cas de l'onde SV) que sur des chemins oedornétriques (cas de l'onde P), au moins après les premiers cycles, empêche l'énergie due aux fréquences élevées d'arriver à la surface.

\section{b. Cas biphasique}

Nous considérons maintenant une colonne de sol élastoplastique saturée d'eau soumise à une excitation harmonique à la base sous la forme d'onde SV. Le matériau est un sable lâche dont les caractéristiques sont présentes dans la thèse de H. MODARESSI (1987). Le 


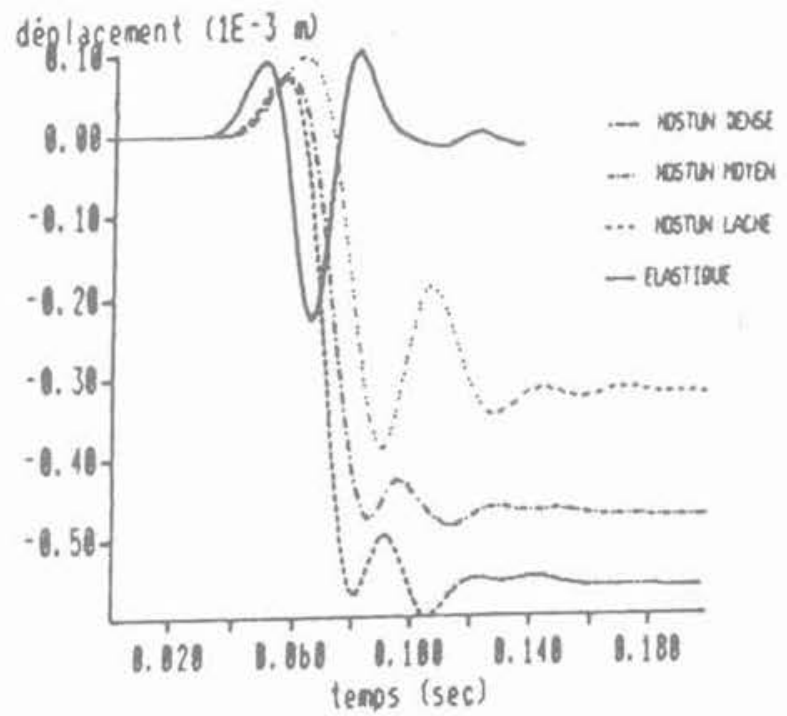

(a) déplacements

(a) displacement

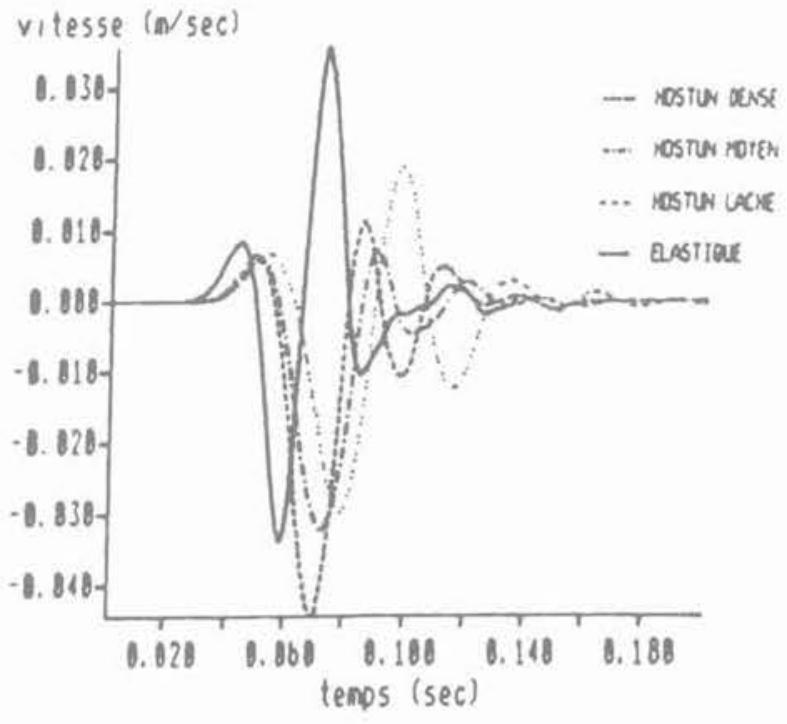

(b) vitesses

(b) velocity

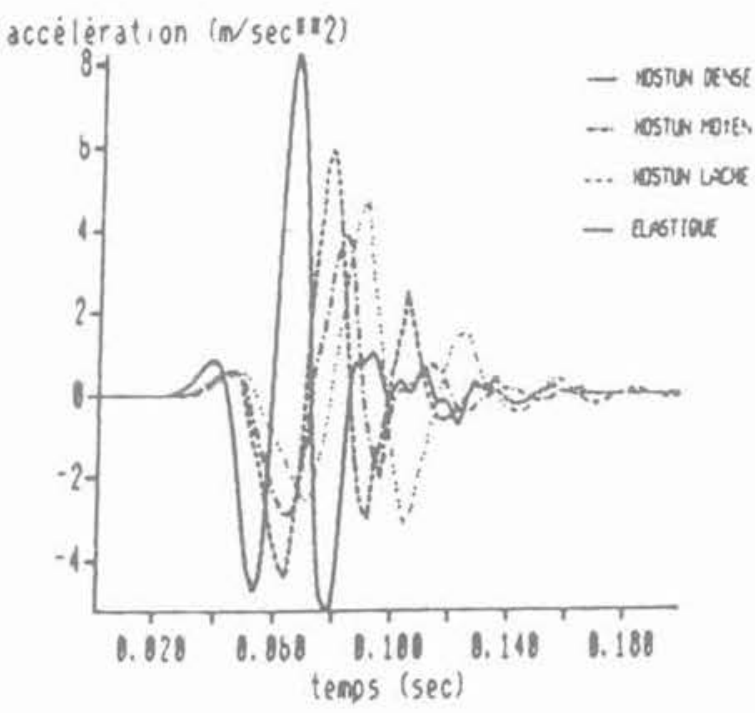

(c) accélérations

(c) acceleration 


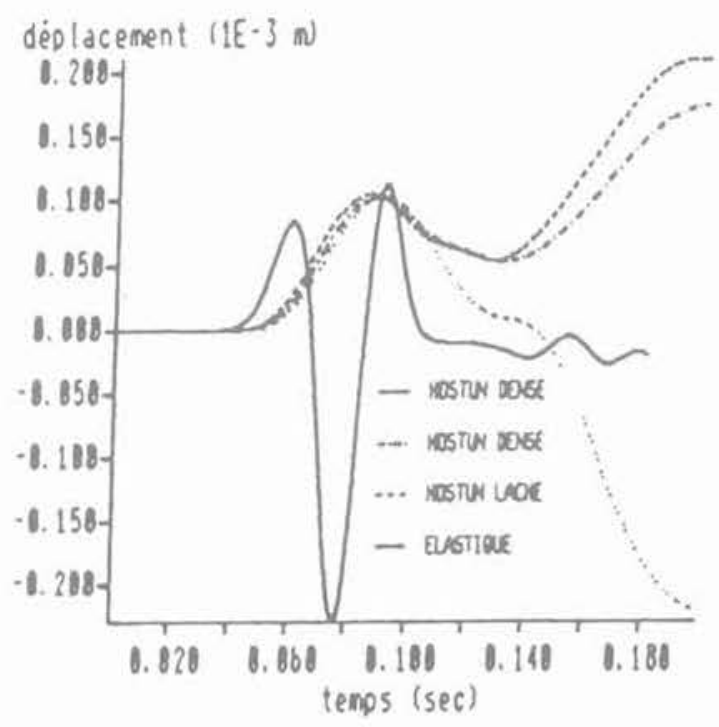

(a) déplacements

(a) displacement

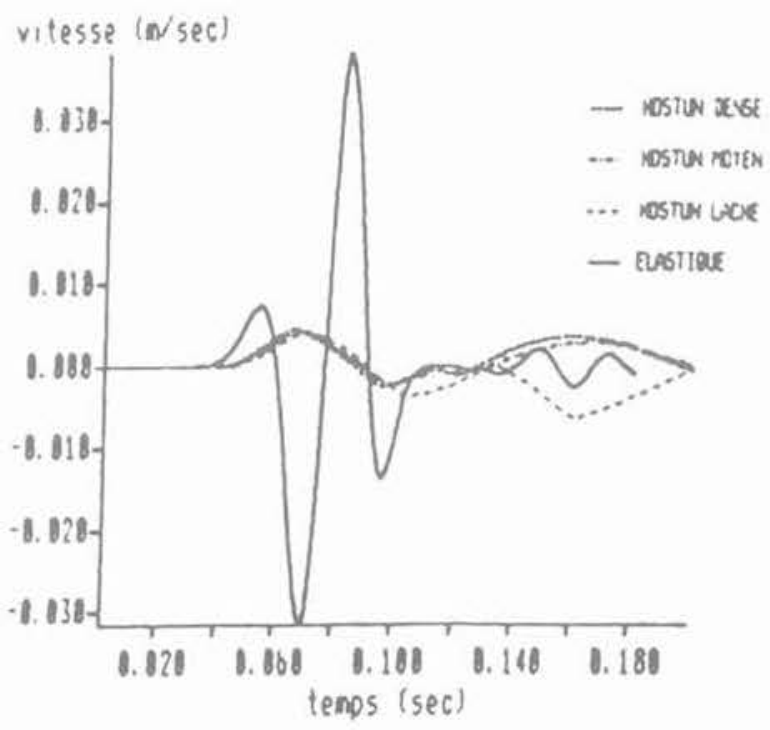

(b) vitesses

(b) velocity

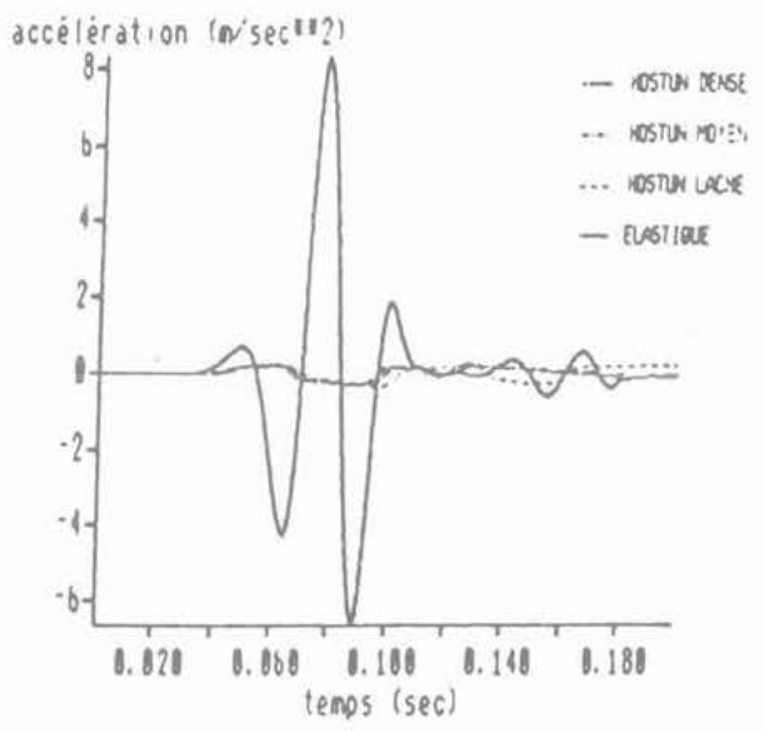

(c) accélérations

(c) acceleration 


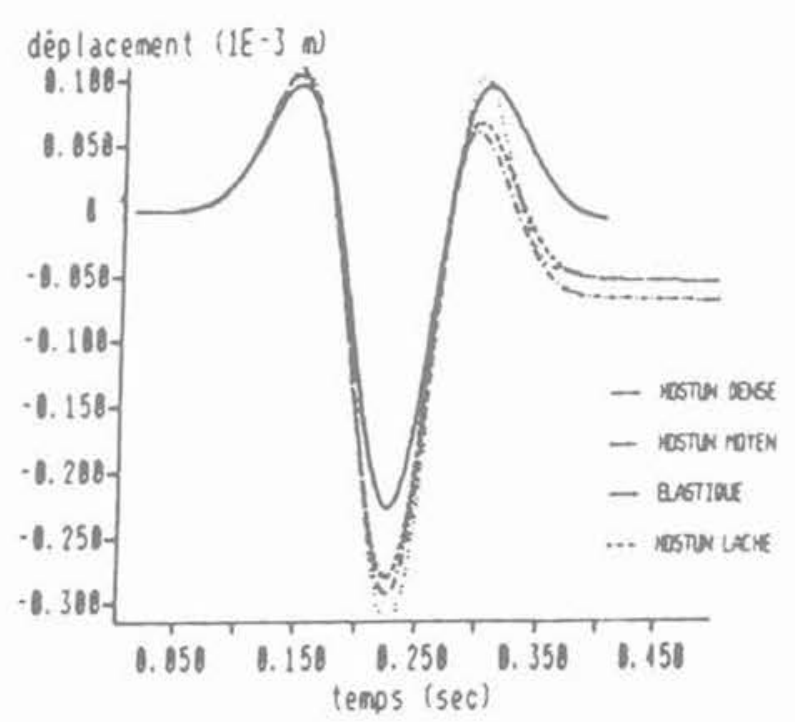

(a) déplacements

(a) displacement

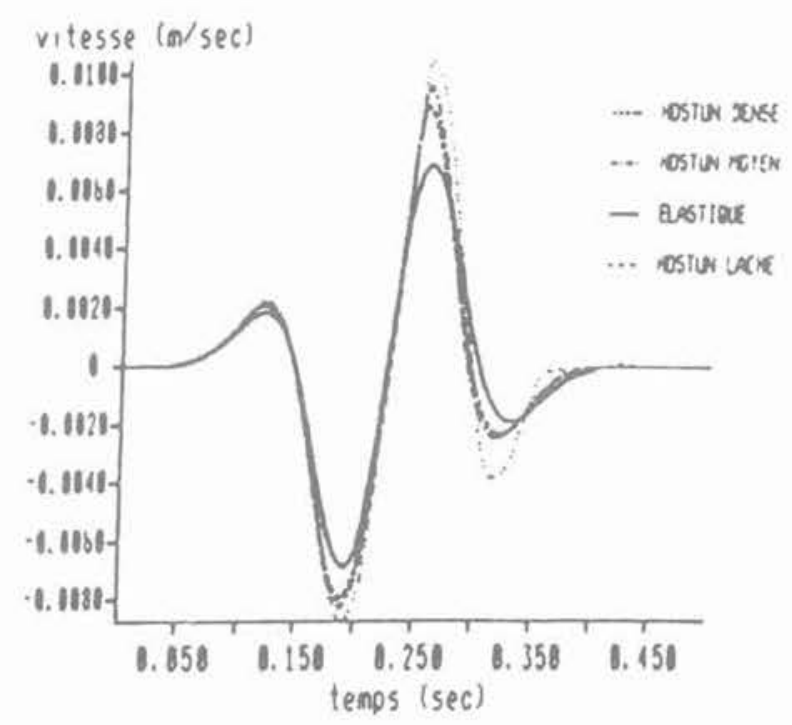

(b) vitesses

(b) velocity

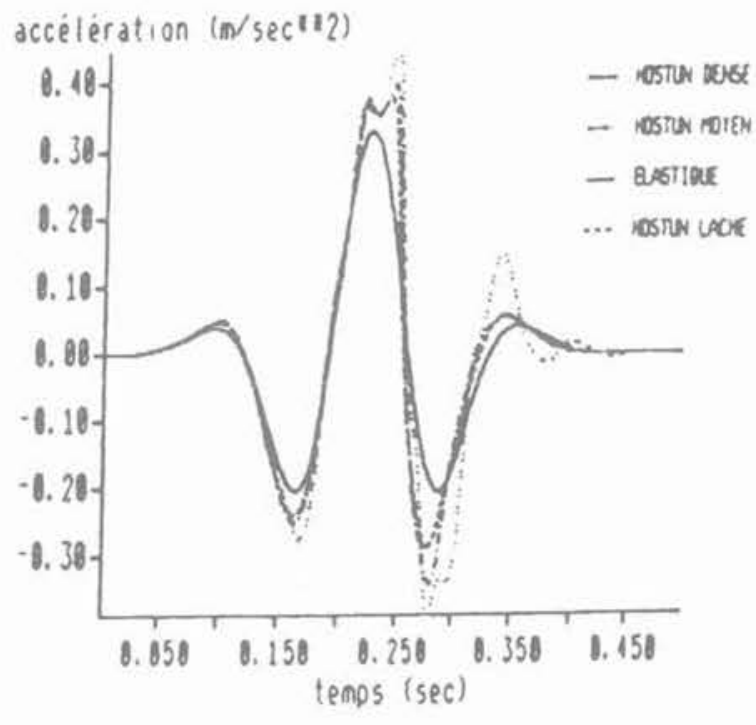

(c) accélérations

(c) acceleration 


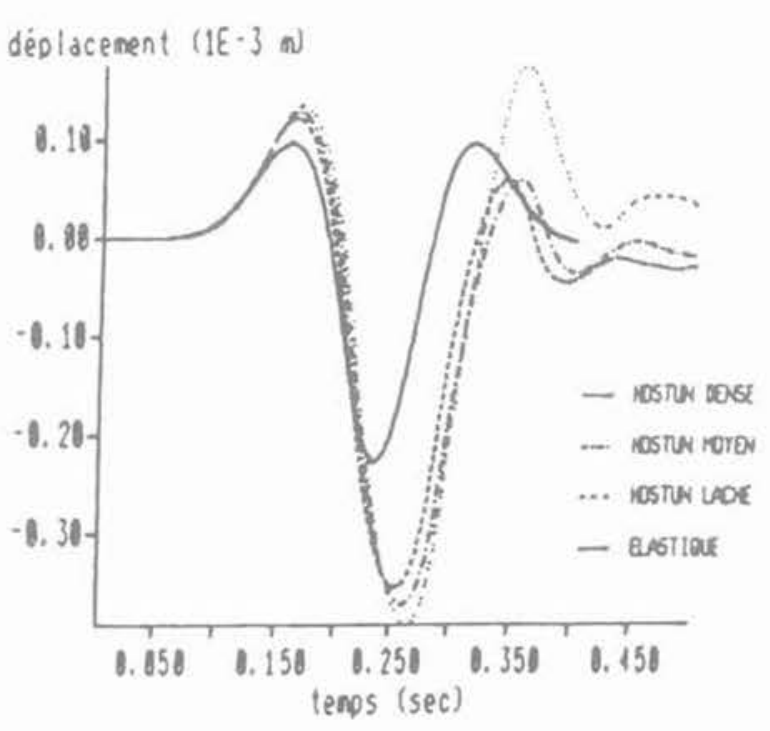

(a) déplacements

(a) displacement

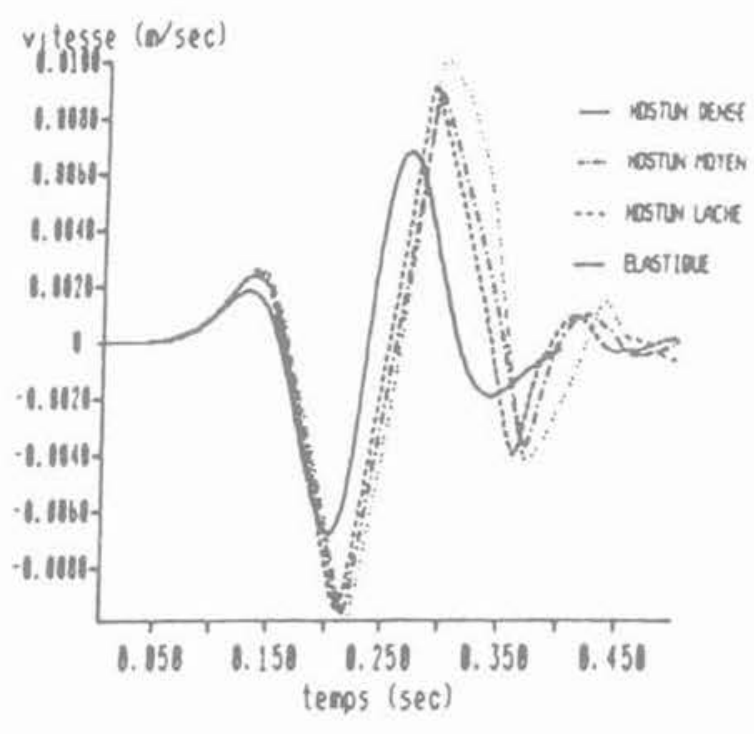

(b) vitesses

(b) velocity

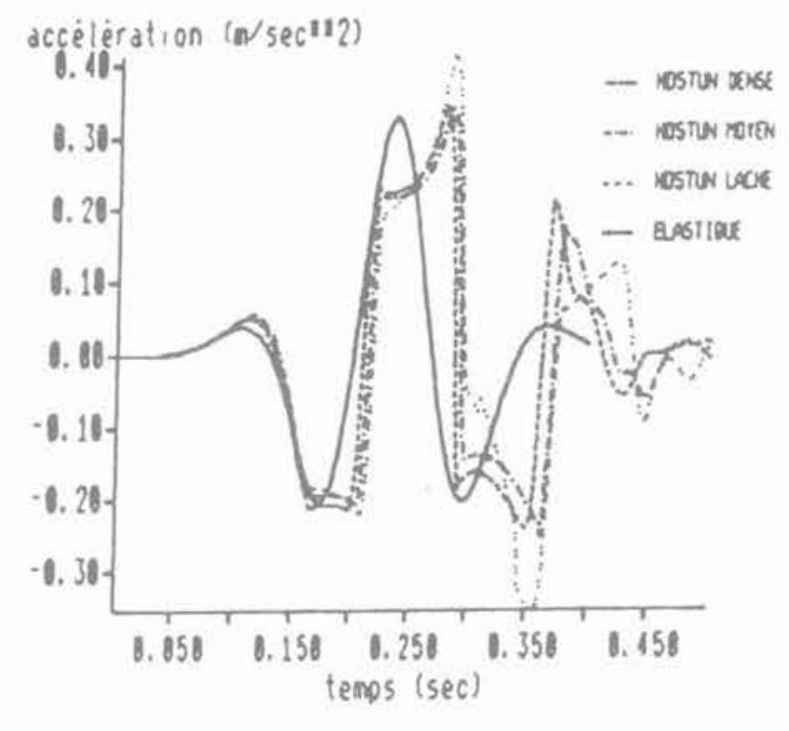

(c) accélérations

(c) acceleration

Fig. 14. - Déplacements, vitesses, accélérations (onde SV/fréquence $5 \mathrm{~Hz}$ ).

Fig. 14. - Displacement, velocity, acceleration (P wave frequency $5 \mathrm{~Hz}$ ). 
tableau 2 donne les paramètres supplémentaires nécessaires pour cette analyse. Les paramètres du schéma de Newmark $\gamma$ et $\beta$ sont choisis respectivement 0,5 et 0,25 , le pas de temps de calcul est de 0,005 secondes et les éléments sont traités implicitement. Le maillage et le champ de contraintes initiales sont présentés sur la figure 15.

Tableau 2 - Caractéristiques du matériau. Table 2 - Material characteristics.

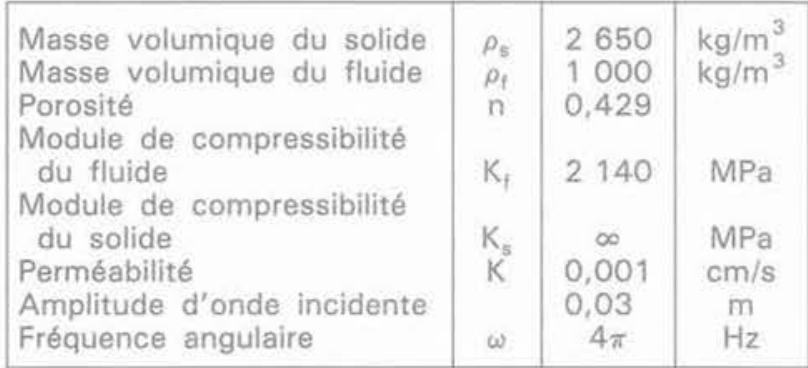

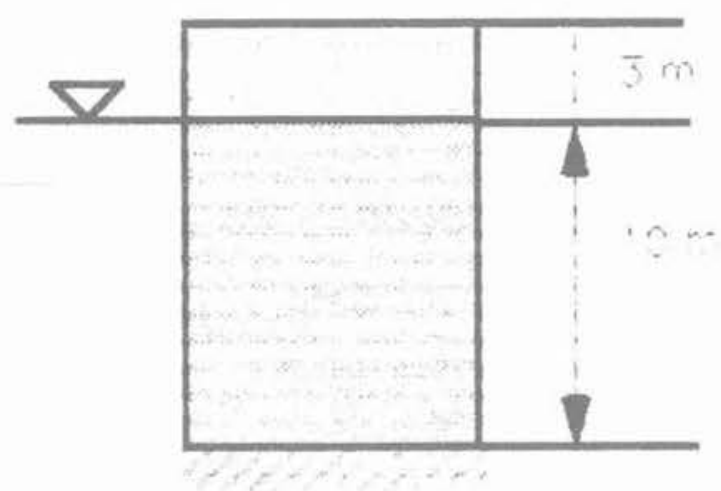

Fig. 15. - Colonne de sol saturé soumise à une onde SV. Fig. 15. - Satured soil column subjected to a SW wave.

L'évolution de la pression interstitielle dynamique dans le temps est donnée pour trois points situés au voisinage de la surface libre de la nappe, à la base et approximativement au milieu de la colonne (fig. 16). On note quau sommet la pression interstitielle reste quasiment nulle à cause des conditions aux limites de drainage. Dans un premier temps, les pressions à la base et au milieu sont quasiment identiques, alors que dû aux niveaux de déformations différentes en ces deux points, elles commencent à diverger ultérieurement. Ce résultat est une manifestation évidente du rôle couplé des variations de volume liées à la dilatance/contractance et de la loi de filtration dynamique. Aucune analyse simplifiée, par exemple non drainée en contraintes totales ne permettrait de reproduire ces phénomènes.

Sur la figure 17, nous avons tracé le profil de la pression interstitielle dynamique sur la hauteur de la colonne, pour quelques étapes du calcul. L'approche et la modélisation de la liquéfaction sont clairement mises en évidence et on voit nettement l'augmentation de la pression interstitielle en profondeur. La courbe représentant la contrainte effective initiale sert de référence, puisque

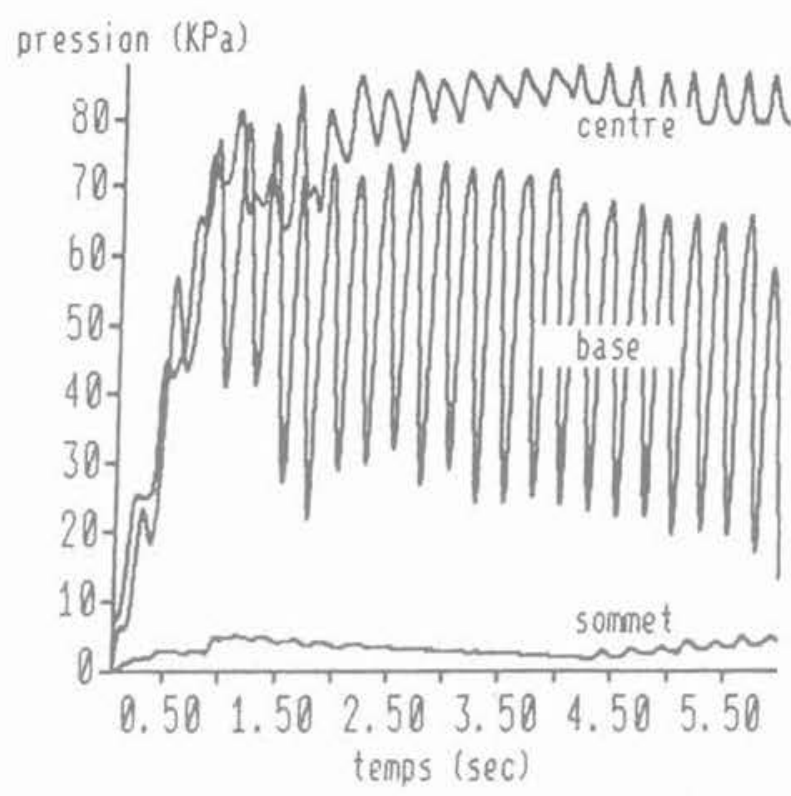

Fig. 16. - Evolution de la pression interstitielle dans le temps. Fig. 16. - Variation of pore pressure with time.

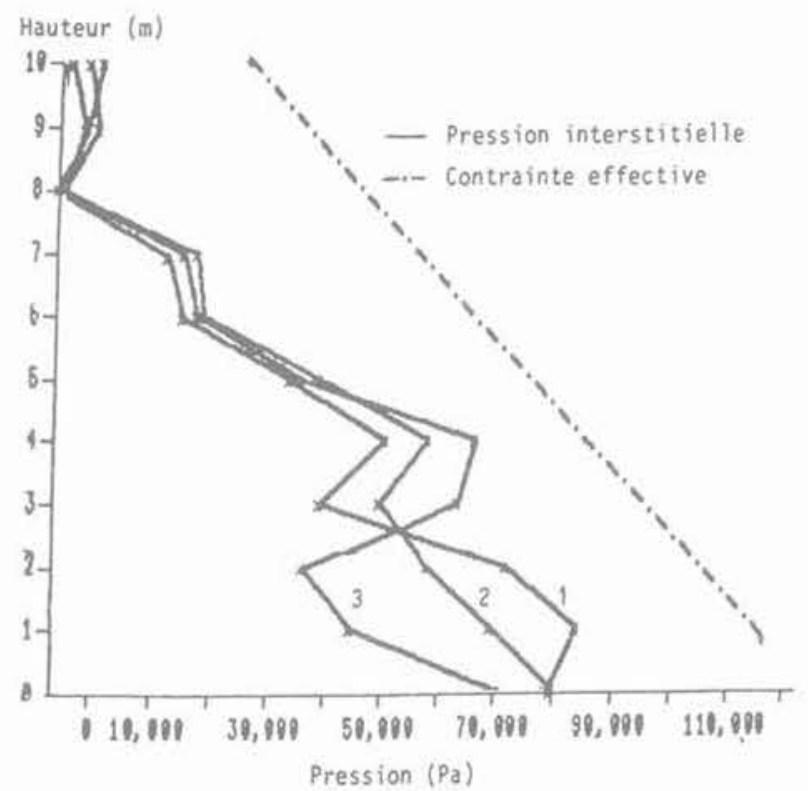

Fig. 17. - Profil de la pression interstitielle sur la hauteur de la colonne.

Fig. 17. - Pore pressure distribution (with the column height versus).

lorsque la liquéfaction est atteinte, si la contrainte totale varie peu, les deux courbes doivent se croiser. Cette dernière hypothèse est assez bien vérifiée en première approximation. 

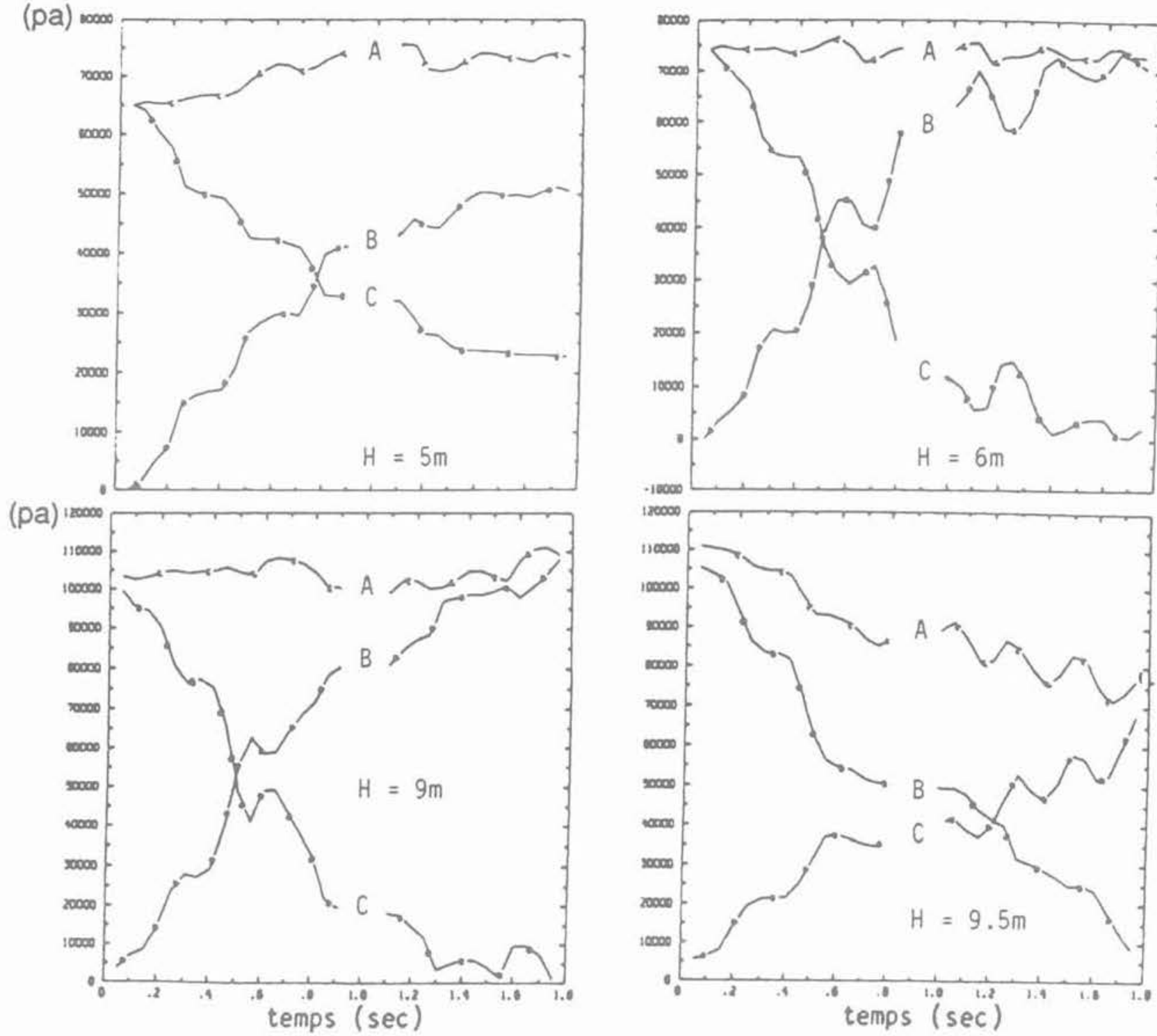

" $\mathrm{A} n$ : contrainte " totale $n\left(\sigma^{\prime}-\Delta_{\mathrm{p}}\right) \propto \mathrm{B} n$ : pression interstitielle dynamique $(\Delta \mathrm{P})$ \& $\mathrm{C} n$ : contrainte effective $\left(\sigma^{\prime}\right)$

Fig. 18. - Contrainte totale, pression interstitielle, contrainte effective à différentes profondeurs.

Fig. 18. - Total stress, pore pressure and effective stress at various depths.

Pour la valeur retenue de la perméabilité (tableau 2), l'augmentation de la pression interstitielle au voisinage de la surface est très faible, au contraire en un point à l'intérieur du massif la pression interstitielle augmente mais la liquéfaction du sol ne se produit pas. Par contre, en diminuant la perméabilité à $5 \times 10^{-6} \mathrm{~cm} / \mathrm{s}$, la liquéfaction survient, comme on peut le voir sur la figure 18. En effet le rôle de la perméabilité sur l'augmentation de la pression interstitielle est indiscutable et cela a été souvent évoqué dans la littérature. La nécessité d'une évaluation précise de la perméabilité des sites constitués du sol saturé et exposés aux mouvements sismiques est très importante pour déterminer les risques de liquéfaction, même si de telles mesures sont bien connues pour leurs difficultés de réalisation.
Nous traçons par la suite pour un point où la liquéfaction se produit, les variations des contraintes de cisaillement en fonction des déformations de cisaillement et de la pression effective moyenne et les variations de la déformation volumique en fonction des déformations de cisaillement (fig. 19). On voit clairement qu'à partir du quatrième cycle la pression effective moyenne tend vers zéro et le sol ne montre plus aucune résistance au cisaillement. On voit également que pendant le chargement la forme des boucles d'hystérésis varie. La nécessité d'une loi de comportement fiable simulant des essais cycliques de contraintes ou déformations à valeurs imposées variables pour évaluer la réponse des sols sous l'effet du chargement sismique est mise en évidence sur des chemins aussi complexes. 


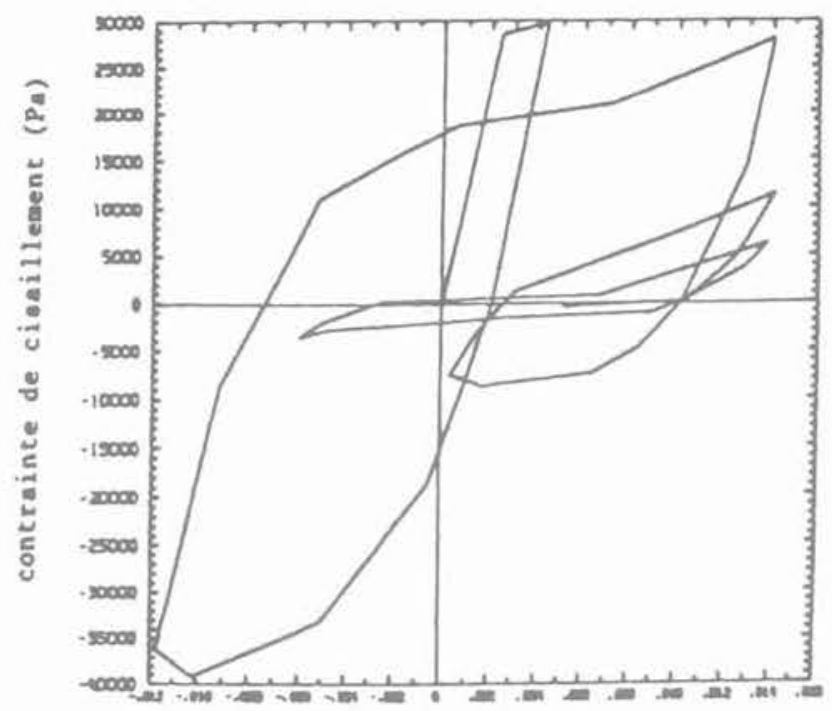

déformation de cisaillement

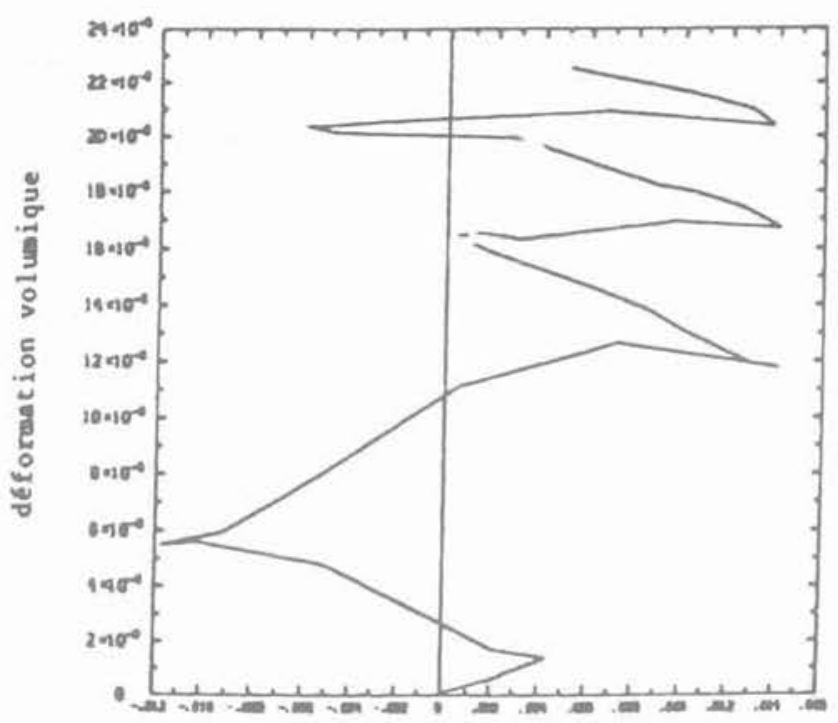

Fig. 19. - Chemins contraintes-déformations. Fig. 19. - Strain-stress relations

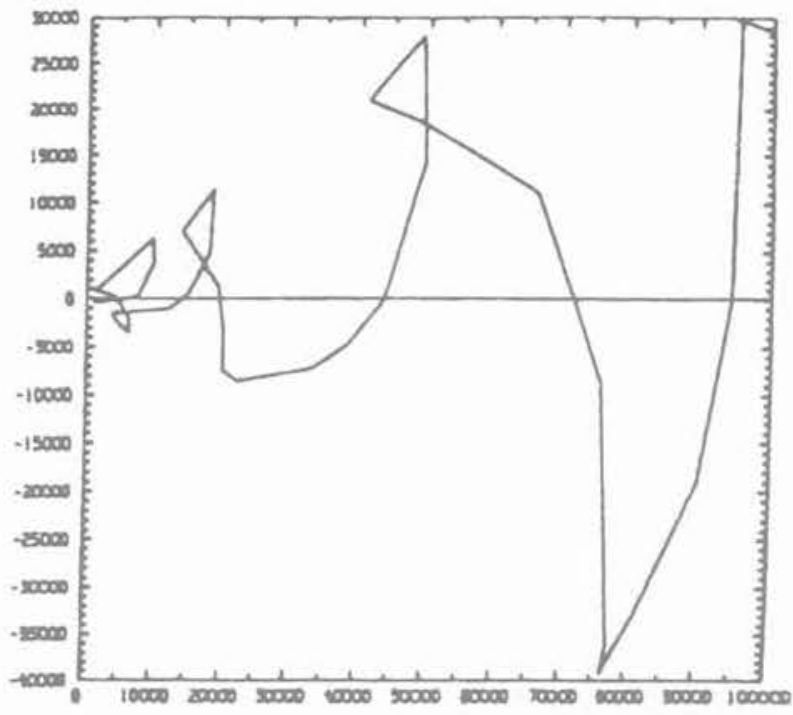

pression woyenne (Pa)

les résultats obtenus dans le cadre d'un comportement élastique linéaire pour l'ensemble de la vallée. Il s'agit d'une vallée alluviale dont la forme et le maillage choisis sont présentés sur la figure 20 .

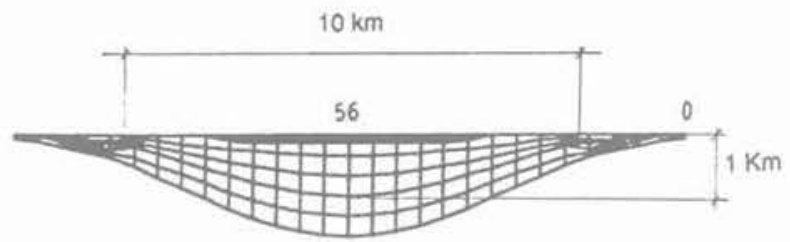

Fig. 20. - Maillage de la vallée.

Fig. 20. - Finite element mesh of the valley.

\section{a. Cas élastique monophasique}

Les caractéristiques des matériaux sont données dans le tableau 3. Les matériaux sont supposés élastiques linéaires. Le pas de temps du calcul, la période caractérisitque du signal incident $\left(t_{p}\right)$ et le temps de l'amplitude maximale ( $\left.\mathrm{t}_{\mathrm{s}}\right)$ sont choisis respectivement égaux à $0,005,2,80$ et 4,0 secondes et tous les éléments sont traités explicitement.

Tableau 3 - Caractéristiques des matériaux.

Table 3 - Material characteristics.

\begin{tabular}{|c|c|c|c|}
\hline & $\begin{array}{l}\text { Bassin } \\
\text { élastique }\end{array}$ & $\begin{array}{c}\text { Substratum } \\
\text { rocheux }\end{array}$ & \\
\hline $\begin{array}{l}\text { Masse volumique } \rho \\
\text { Module de Young } \\
\text { Coefficient } \\
\text { de Poisson }\end{array}$ & $\begin{array}{c}2000 \\
2,6110^{3} \\
0,33\end{array}$ & $\begin{array}{c}3300 \\
1,0110^{5} \\
0,25\end{array}$ & $\underset{\mathrm{MPa}}{\mathrm{kg} / \mathrm{m}^{3}}$ \\
\hline
\end{tabular}

Nous abordons maintenant les effets de site et les amplifications locales dues à la géométrie des vallées alluviales, c'est-à-dire essentiellement les effets bidimensionnels sur le champ libre. L'onde incidente est une onde plane se propageant verticalement sous la forme d'une onde de cisaillement (SV). Notre code de calcul par l'intermédiaire des éléments de frontières nous permet la prise en compte des incidences non nulles, mais ici nous reprenons l'exemple présenté par BARD et $\mathrm{BOUCHON}$ (1980), qui nous servira de référence, pour comparer 
H56

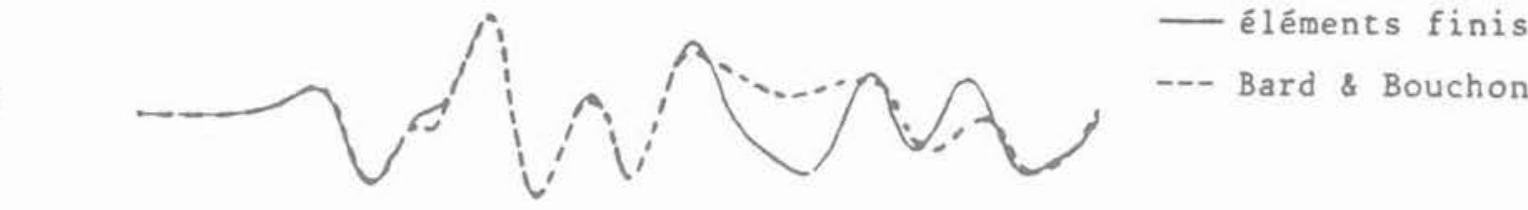

- éléments finis

H28

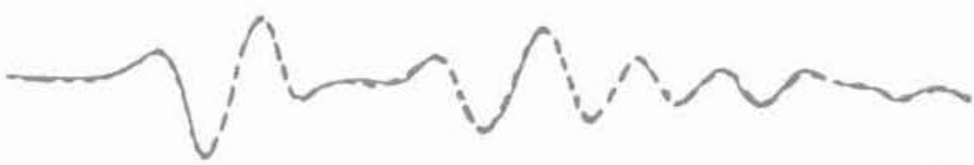

$\mathrm{H} \mathrm{O}$

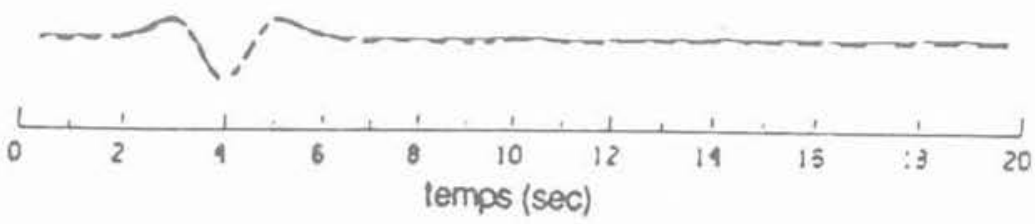

Fig. 21. - Comparaison avec Bard \& Bouchon.

Fig. 21. - Comparaison of finite of element solution with Bard and Bouchon.

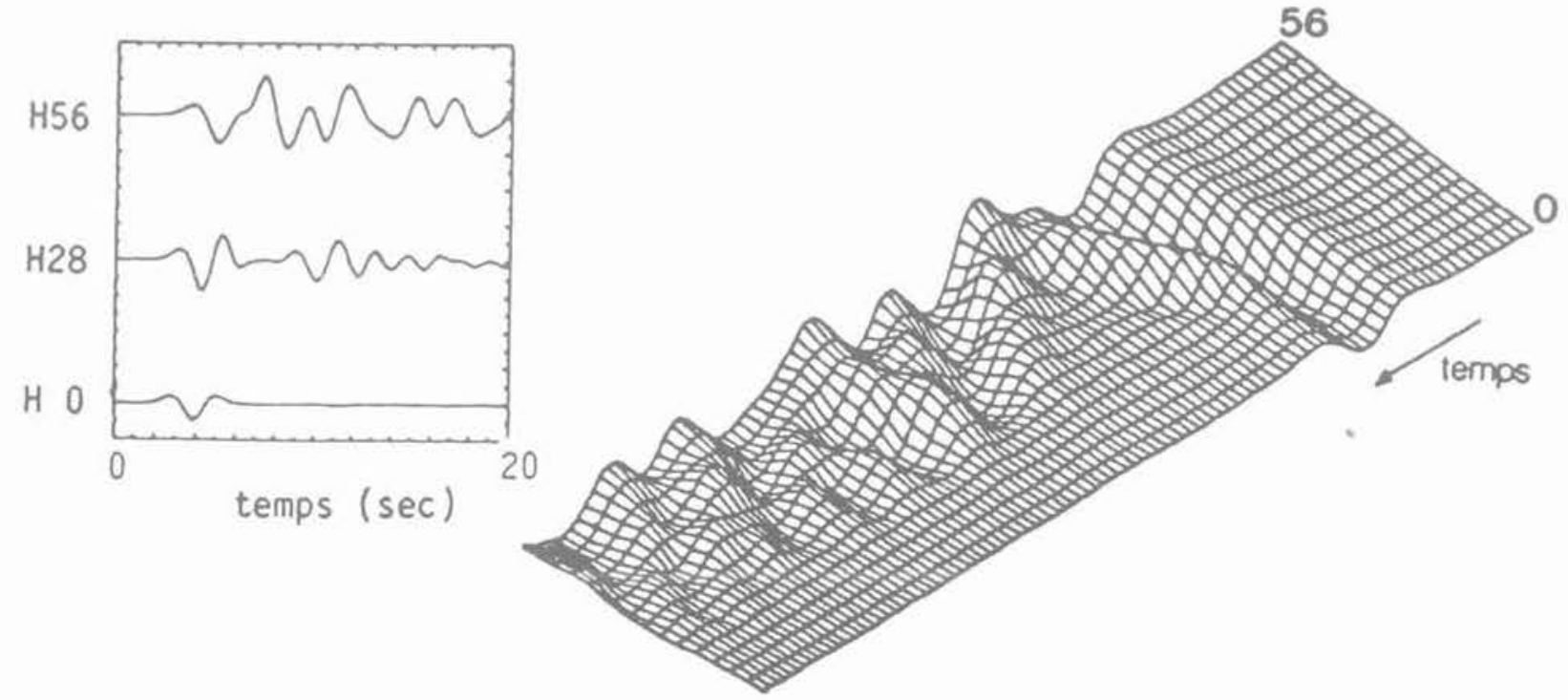

Fig. 22. - Déplacements horizontaux en surface (onde SV verticalement incidente).

Fig. 22. - Surface horizontal displacement time history (for a vertically incident SV wave). 


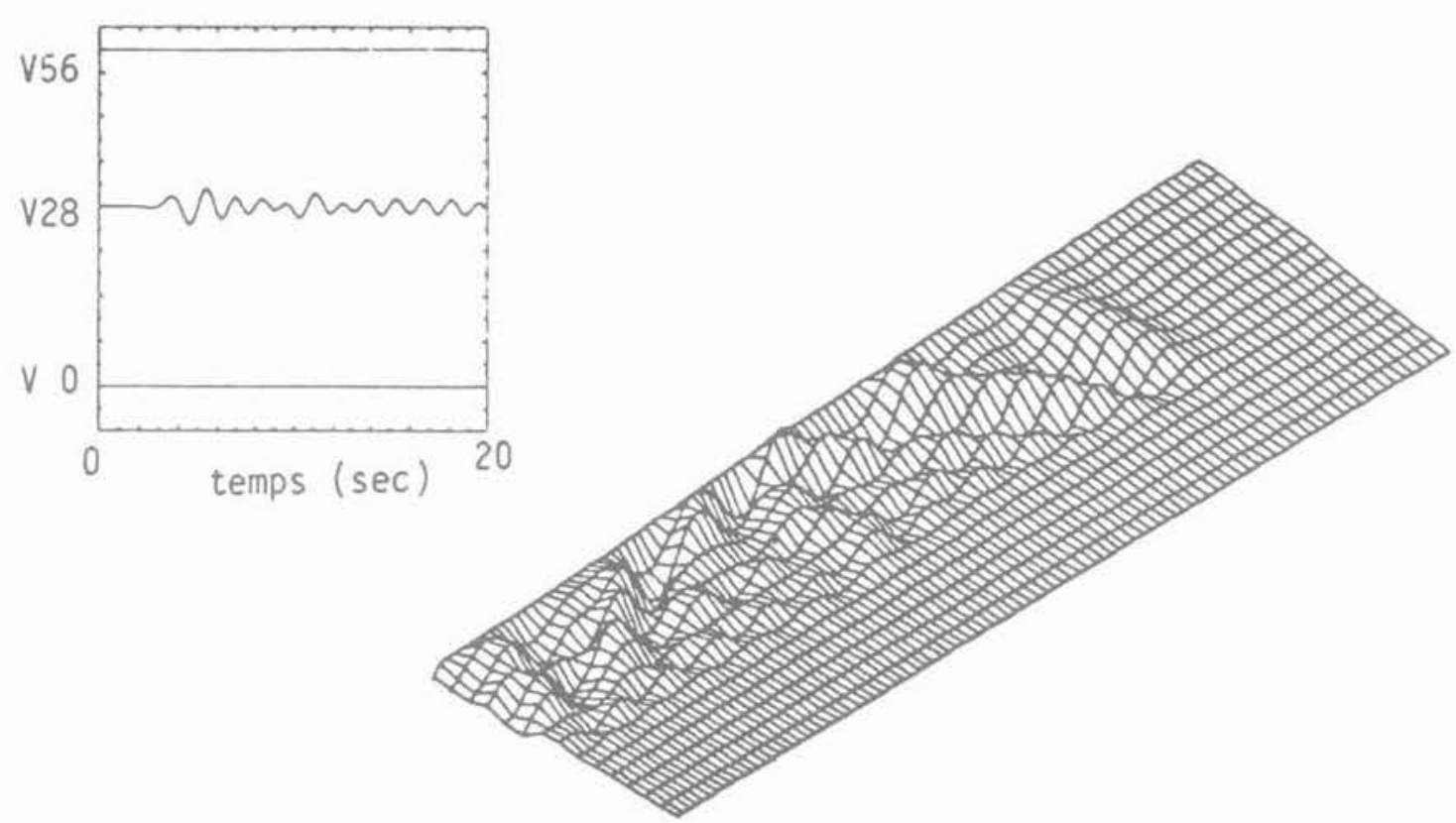

Fig. 23. - Déplacements verticaux en surface (onde SV verticalement incidente),

Fig. 23. - Surface vertical displacement time history (for a vertically incident SV wave).

Sur la figure 21, nous présentons la comparaison entre les résultats obtenus pour trois points de la surface libre respectivement au milieu, à l'extrémité et à mi-distance entre ces deux points avec ceux présentés par BOU. $\mathrm{CHON}$ et $\mathrm{BARD}$. On peut constater que la concordance des résultats pour le point au centre de la vallée est moins bonne que pour les deux autres points: Cependant on observe le même nombre de pics et la même valeur maximale suivant les deux méthodes.

Les résultats obtenus pour les champs de déplacements à proximité de la surface sont présentés sur les figures 22 et 23. On peut observer que l'onde SV incidente arrivant à la surface crée des ondes de surface (en particulier les ondes de Rayleigh) dans les coins de la vallée qui, se propageant sur la surface, amplifient les déplacements qui persistent quelques temps après le passage du signal. La création de déplacements verticaux n'aurait pu être mise en évidence avec une modélisation monodimensionnelle. L'amplification maximale des déplacements horizontaux se produit au centre de la vallée où les ondes de surface créées aux deux coins de la vallée se rencontrent et elle est approximativement 4 à 5 fois plus grande par rapport à ce qu'on pouvait avoir en l'absence d'irrégularité géométrique. Cela confirme la nécessité des calculs bi- voire tri-dimensionnels.

On observe à la surface l'apparition d'une onde de Rayleigh associée à un mouvement elliptique rétrograde à prédominance horizontale qui s'amortit rapidement avec la profondeur (fig. 24).

L'influence de l'amplitude et la durée du mouvement, ainsi que le contenu fréquentiel sont bien présentés par le spectre de réponse. On peut observer que les accélérations verticales sont négligeables au centre et à l'extrémité de la vallée, que l'accélération maximale horizontale est presque trois fois plus grande au centre de la

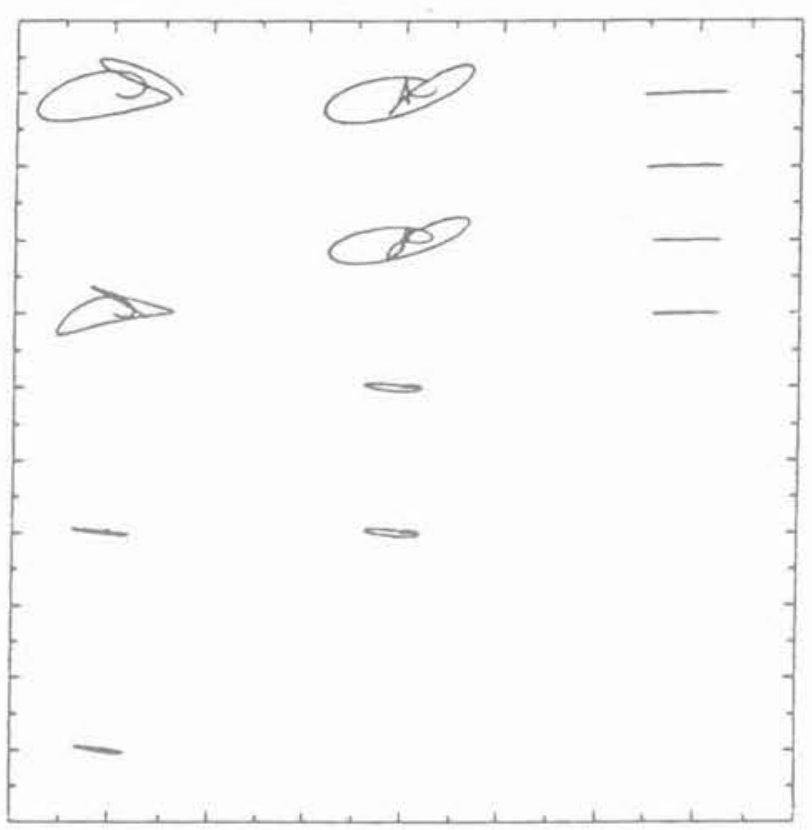

Fig. 24. - Trajectoires en surface et en profondeur.

Fig. 24. - Particle motion al a given time for differents points on the surface and their evolution with depth

vallée par rapport à l'extrémité tandis que la fréquence de pic reste sensiblement la même.

Enfin les maillages déformés de la partie droite de la vallée alluviale sont présentés dans la figure 25 et montrent que le mouvement se prolonge essentiellement à l'intérieur de la vallée et s'amplifie à proximité de la surface libre. 


\section{$t=5$ secondes}

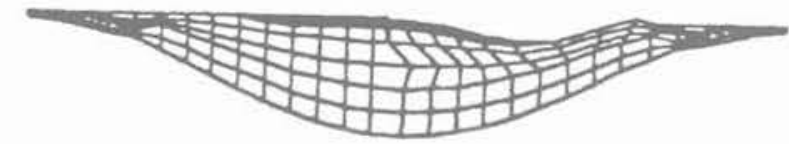

$l=10$ secondes

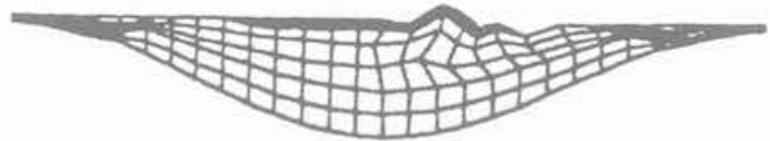

$t=15$ secondes

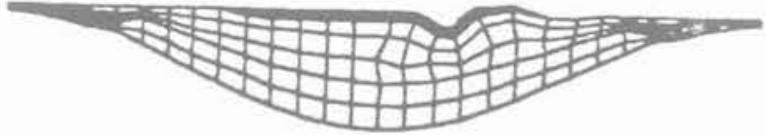

$t=20$ secondes

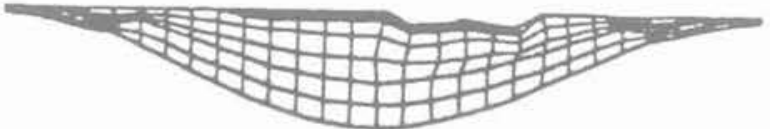

Fig. 25. - Maillage déformé de la partie droite de la vallée. Deformed mesh of the right hand side of the valley.

Ces tests montrent l'importance de la prise en compte des hétérogénéités latérales. On peut constater que le mouvement n'est pas seulement amplifié mais également prolongé dans le temps. Ceci est très important dans le comportement des ouvrages construits sur ces sites et également dans le comportement des sols constituant les couches superficielles, car par exemple dans les conditions non drainées l'augmentation du nombre de cycles ainsi créés peut les amener jusqu'à la liquéfaction.

\section{b. Cas anélastique monophasique}

Dans cette section nous présentons les résultats numériques obtenus pour la même vallée alluviale en tenant compte du comportement réel, élastoplastique des sols constituant les couches superficielles de la vallée. Nous étudions la réponse de la vallée soumise à une onde de cisaillement SV verticalement incidente et sous la forme d'un signal de Ricker. La forme et les dimensions de la vallée, ainsi que les caractéristiques du champ incident sont celles présentées précédemment,

La forme de la vallée, le contraste d'impédance entre la vallée et le milieu avoisinant sont des facteurs prédominant sur les mouvements sismiques à la surface de celle-ci. Ici, pour mettre l'accent sur l'influence du comportement du sol, nous maintenons ces deux paramètres ainsi que les caractéristiques du champ incident constants et nous renvoyons à BARD (1983) pour une étude paramétrique sur l'influence des divers paramètres. Le matériau choisi est le sable láche d'Hostun déjà étudié précédemment. Les ondes rayonnées vers l'extérieur sont évacuées par l'intermédiaire des éléments paraxiaux et le seul amortissement existant dans le milieu est dû au comportement plastique du matériau pris en compte par la loi de comportement. Les paramètres du schéma de Newmark $\gamma$ et $\beta$ sont chosis respectivement 0,5 et 0,25 , le pas de temps de calcul est de 0,005 secondes et les éléments sont traités implicitement dans le domaine anélastique et explicitement partout ailleurs.

Les déplacements sont présentés sur la figure 26 . On peut constater que dans la partie anélastique les résultats sont nettement différents par rapport aux calculs précédents. On observe une nette amplification des mouvements horizontaux et verticaux et accompagnés par des déformations irréversibles. On constate en particulier l'apparition d'un tassement important dans ces matériaux. Le sable lâche sous la vibration produite par le mouvement prolongé des ondes prises en piège dans la vallée se densifie.

Les ondes réfléchies sur les bords de la vallée créent encore des ondes de surface ayant un mouvement elliptique rétrograde ressemblant aux ondes de Rayleigh. Cependant il faut noter que la propagation des ondes dans les milieux non linéaires est très complexe. On peut néanmoins préciser que par rapport au calcul élastique, on constate que les mouvements verticaux sont accentués. On remarque néanmoins que l'existence du bassin anélastique ne modifie pas les mouvements des zones voisines de manière significative.

Les spectres de réponse montrent qu'à part l'amplification importante en particulier pour les mouvements verticaux, il $y$ a des accélérations très marquées qui se produisent dans les domaines de longues périodes et qui étaient absentes dans les spectres obtenus par une analyse élastique. Ceci montre l'intérêt d'une modélisation précise du comportement des sols pour ce genre de calcul. Traditionnellement, dans les spectres proposés pour le dimensionnement des structures, on tient compte de linfluence des sols mous sur la prolongation du mouvement sur les périodes plus grandes (SEED et IDRISS, 1982), mais en général les amplifications dues à ces matériaux sont plus ou moins sous-estimées. Ceci peut être tout à fait acceptable pour les mouvements 


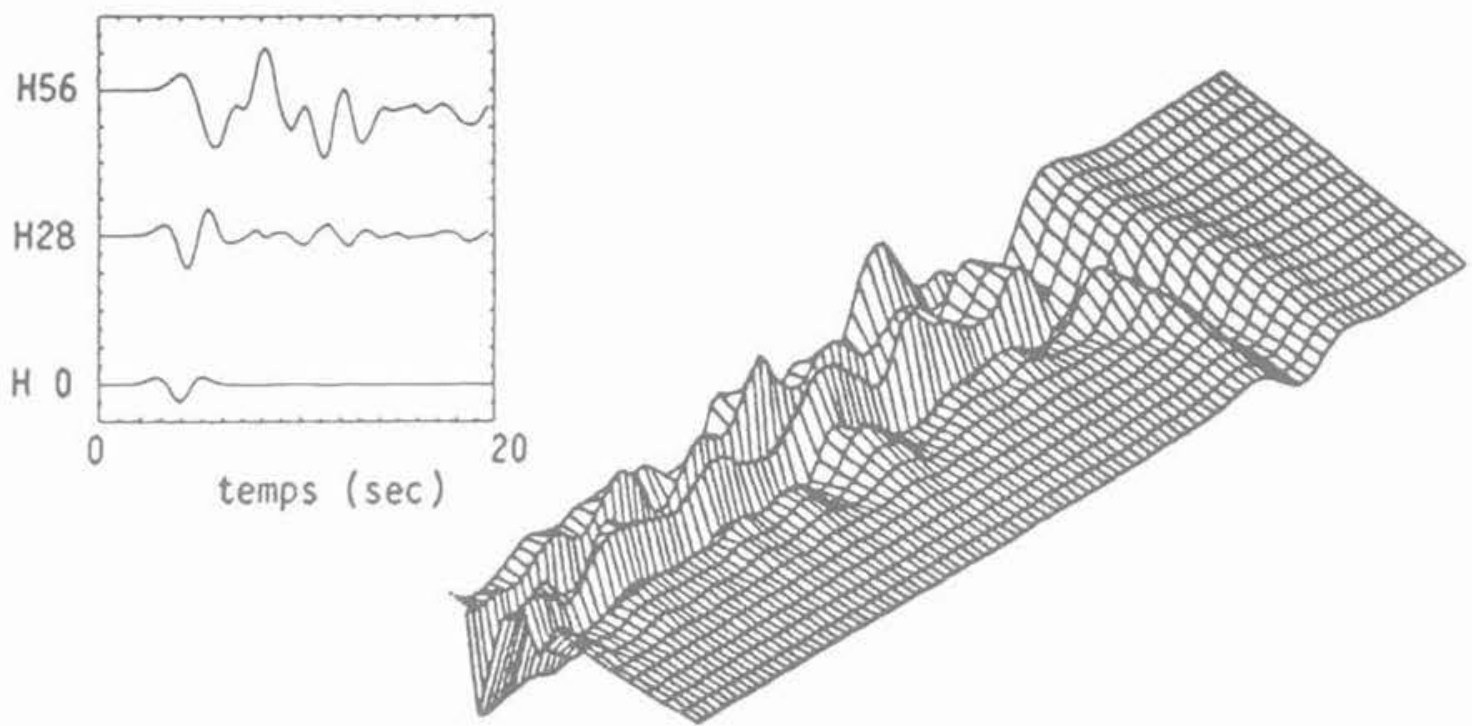

Fig. 26. - Déplacements horizontaux à la surface (sable lâche sec).

Fig. 26. - Surface horizontal displacement time history (for a dry loose sand).

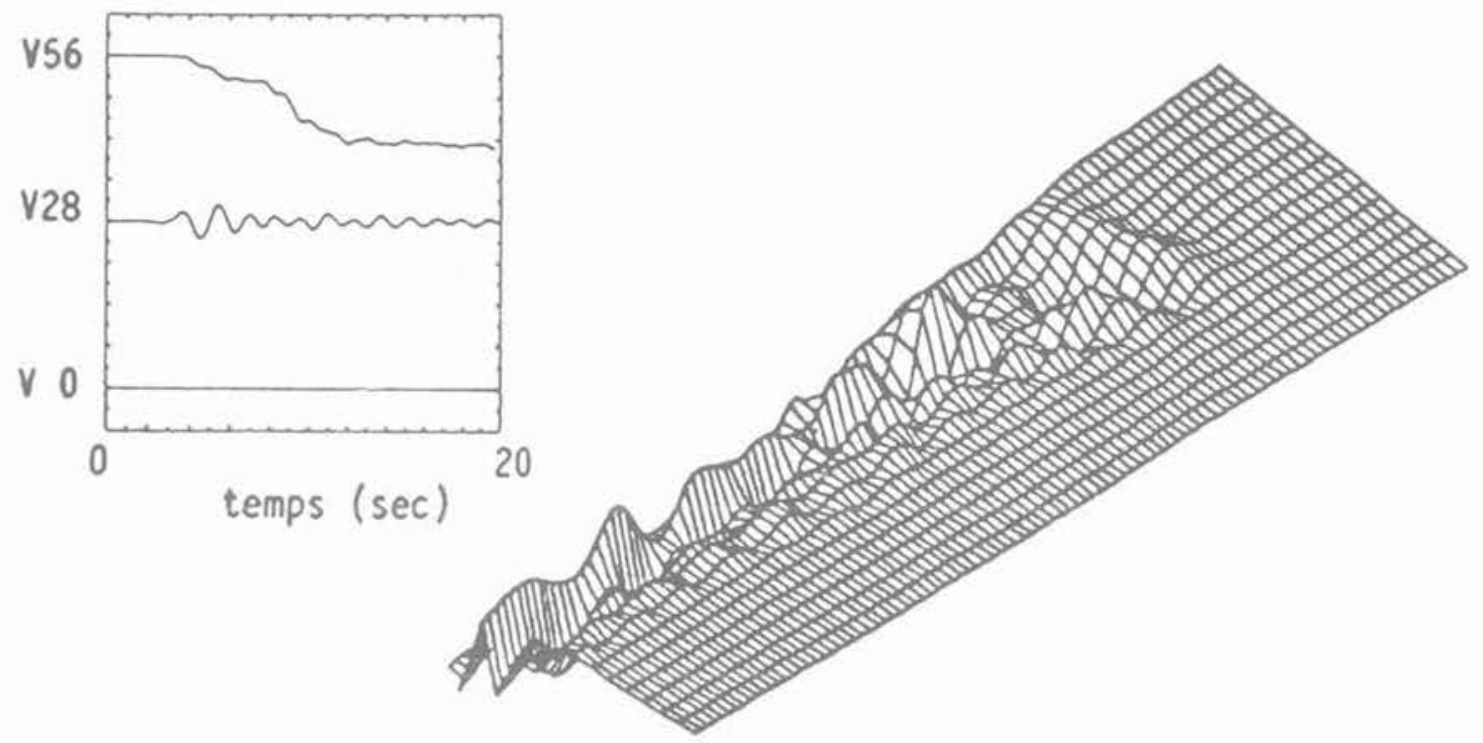

Fig. 27. - Déplacements verticaux à la surface (sable lâche sec).

Fig. 27. - Surface vertical displacement time history (for a dry loose sandi.

relativement faibles, mais semble sujet à caution pour les séismes d'intensité très forte:

Pour voir linfluence de la densité sur le comportement du sable pendant le séisme nous avons effectué la même analyse que précédemment avec cette fois un sable dense avec une porosité de 0,36. Les résultats (MODARESSI, 1987) montrent que, vue l'intensité du champ sismique, une densification se produit malgré la nature dense du sable choisi, évidemment plus faible que dans le cas précédent. Les déformations permanentes sont plus petites en ce qui concerne les mouvements horizontaux et l'accélération horizontale maximale est légèrement plus forte. En quelque sorte, le matériau étant plus raide, son comportement s'approche d'avantage d'un comportement élastique.

\section{c. Cas anélastique biphasique}

Dans les exemples précédents les matériaux étudiés étaient secs. Nous procédons maintenant à une étude sismique complète d'une vallée alluviale constituée d'un bassin de sable lâche saturé d'eau. La zone élastique qui entoure ce bassin est aussi considérée saturée et l'ensemble repose sur un substratum élastique sec. A l'interface entre cette zone et le substratum, un drainage parfait est permis. On notera ici, l'intérêt du choix retenu des conditions aux limites et de la formulation variationnelle, per- 


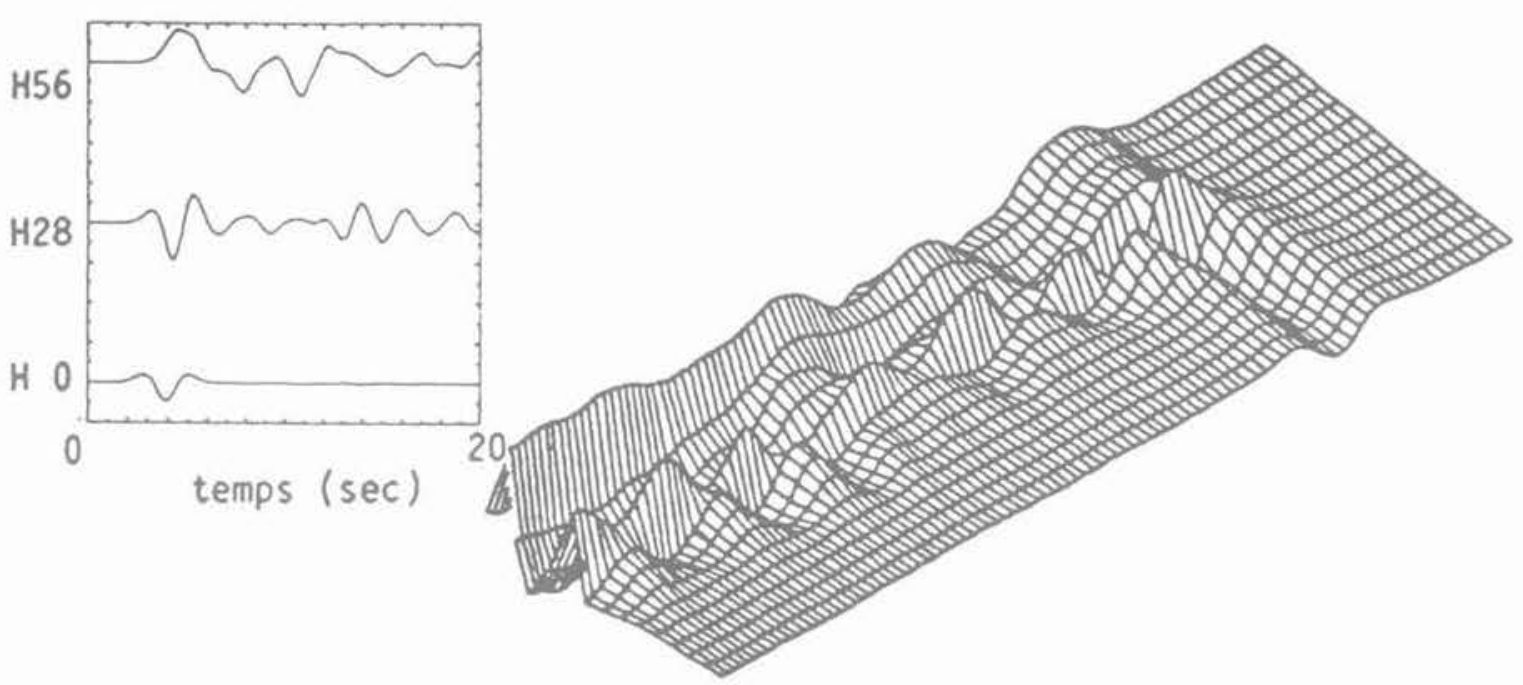

Fig. 28. - Déplacements horizontaux en surface (sable lâche saturé).

Fig. 28. - Surface horizontal displacement (for a saturated loose sand).

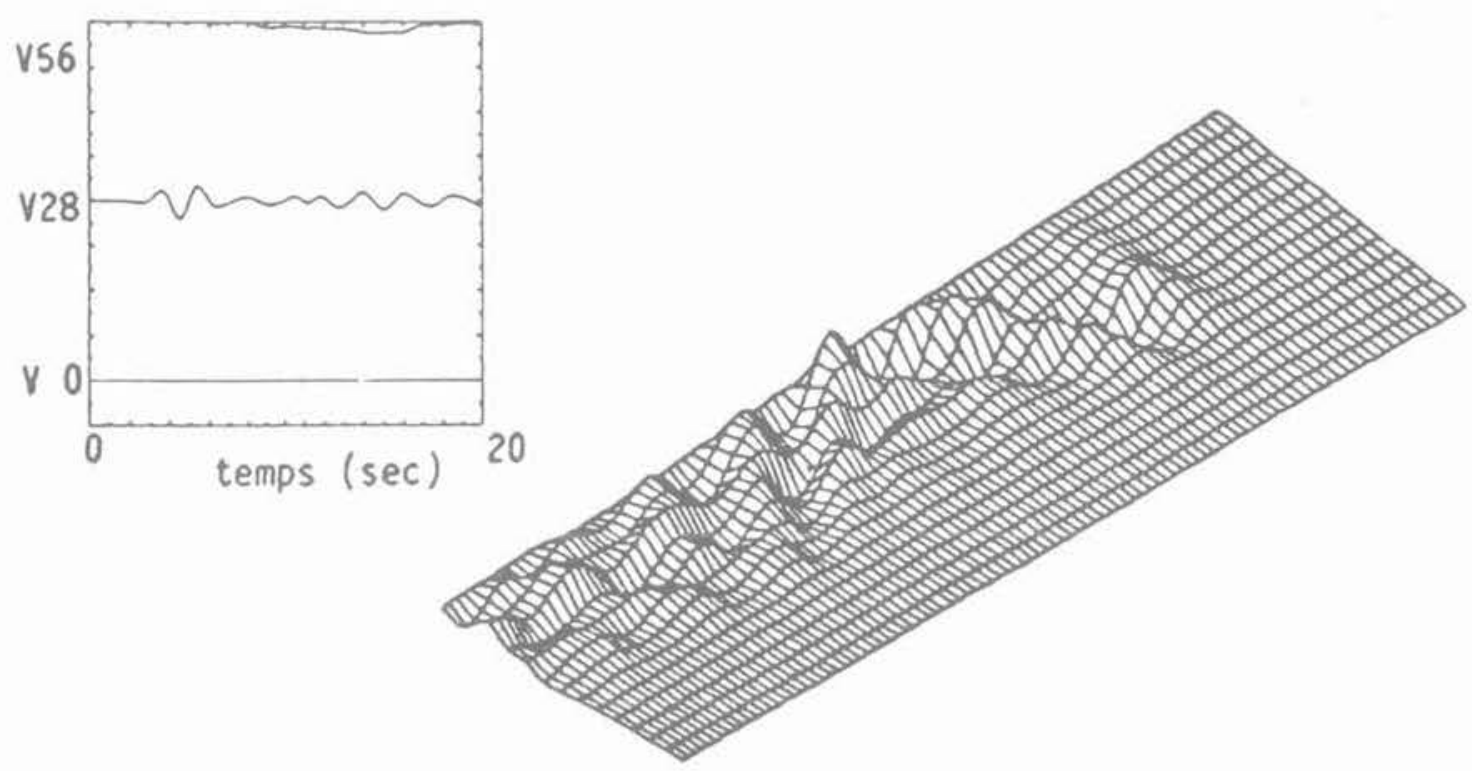

Fig. 29. - Déplacements verticaux en surface (sable lâche saturé).

Fig. 29. - Surface vertical displacement (for a saturated loose sand).

mettant de juxtaposer des zones biphasiques et monophasiques.

La géométrie de la vallée, les caractéristiques du champ incident et les propriétés des sols sont celles utilisées dans les exemples précédents. La porosité du sable est de 0,43 . Les paramètres du schéma de Newmark $\gamma$ et $\beta$ sont choisis respectivement 0,5 et 0.25 , le pas de temps de calcul est de 0,005 secondes et les éléments sont traités implicitement dans les domaines biphasiques et explicitement dans les autres zones du maillage.

Les figures 28 et 29 montrent les champs des déplacements à la surface de la vallée. On peut constater que les mouvements, en particulier les accélérations sont amorties par rapport au calcul monophasique équivalent. Le tassement vertical empêché par la condition quasi non drainée, due au chargement très rapide du champ sismique est substitué par une liquéfaction locale d'un certain nombre de points se situant de 20 à 40 mètres de profondeur qui se généralise petit à petit jusqu'à la liquéfaction d'une zone à environ 120 mètres de profondeur. Les profils de la variation de la pression interstitielle sur l'axe vertical passant par le centre de la vallée sont présentés sur la figure 30 . On constate que vers 14 secondes après le début du chargement sismique il y a une montée très nette de la pression interstitielle dans les points situés à 120 mètres de la surface libre. 


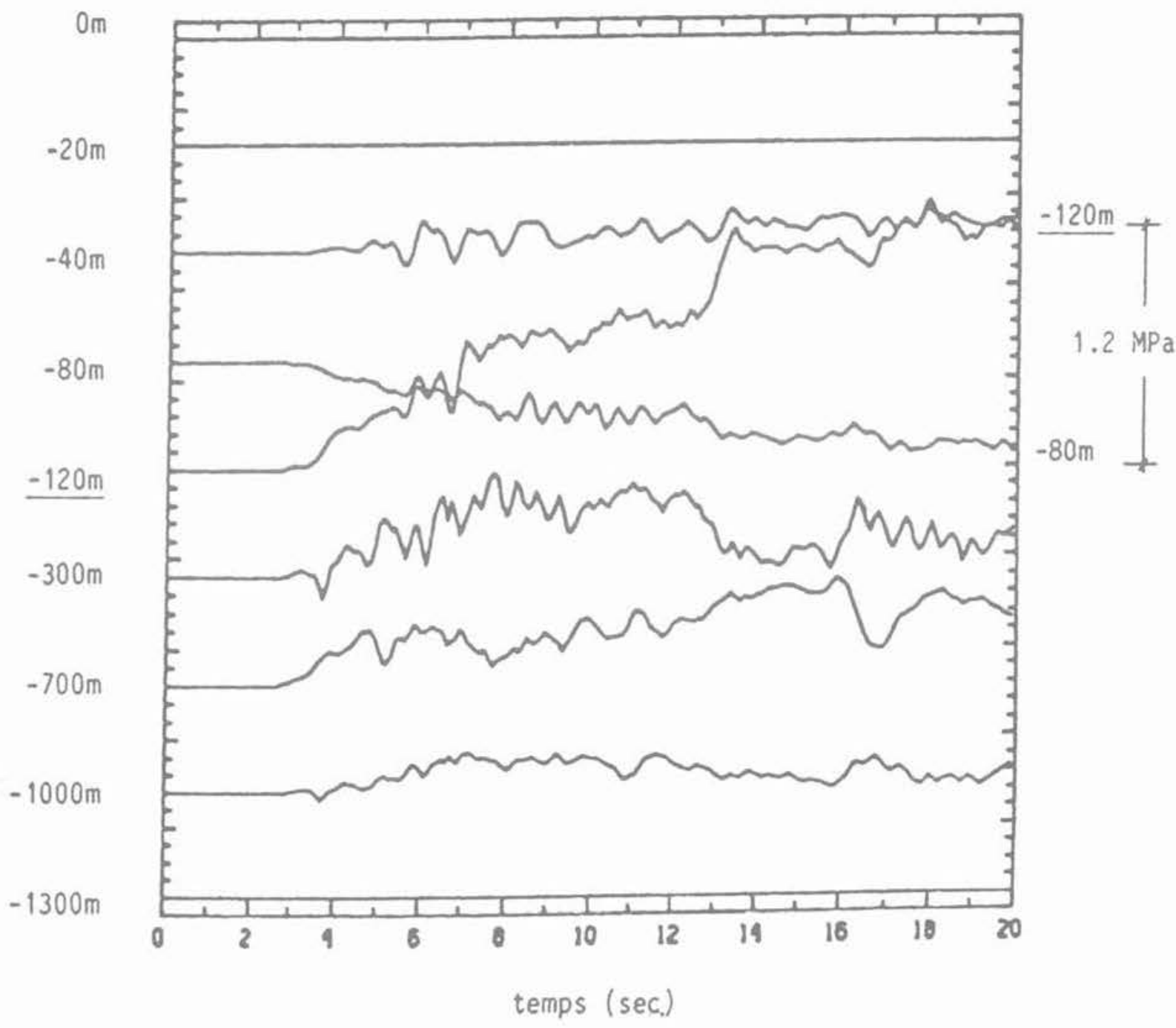

Fig. 30, - Pression interstitielle sur un axe vertical au centre de la vallée. Fig. 30. - Excess pore pressure at various depths along the axis of the valley.

Il faut souligner que dans l'exemple traité nous avons supposé que le sol constituant le bassin anélastique a les mêmes propriétés sur toute la profondeur de bas$\sin$. En pratique dans les sites alluviaux on observe que la densité du sol augmente avec la profondeur, et que les zones liquéfiables dépassent rarement une profondeur supérieure à 50 mètres. Des courbes typiques contraintes-déformations prennent une allure peu habituelle (MODARESSI, 1987). On constate néanmoins que malgré le chemin de chargement très complexe, le sol progresse vers un état de liquéfaction, où les contraintes effectives s'annulent.

Le calcul des spectres de réponse montrent à quel degré la réponse du sol a été modifiée par la présence de l'eau. Nous avons indiqué ci-dessus la diminution importante de l'amplitude des accélérations obtenues à la surface du sol et cela change évidemment la valeur maximale de l'accélération dans le spectre de réponse. Par ailleurs le contenu fréquentiel a été largement modifié, En ce qui concerne les composantes horizontales, le mouvement s'étale sur une gamme large de fréquence par rapport au cas monophasique, et donne lieu à plu- sieurs pics successifs regroupés autour de la fréquence de pic du champ incident. Pour les composantes verticales, on constate toujours des valeurs plus faibles que dans le cas monophasique, mais cette fois le contenu fréquentiel est encore plus affecté par la présence de leau, car les pics sont translatés à des fréquences plus élevées. Des ondes de surface se produisent comme dans les exemples précédents et prolongent le mouvement entrainant la liquéfaction du sol.

\section{BIBLIOGRAPHIE}

AUBRY D., HUJEUX J.C., LASSOUDIERE F., MEIMON Y. (1982), A double memory model with multiple mechanisms for cyclic soil behaviour. Int. Symp. Num. Mod. Geomech., Balkema, 3-13.

AUBRY D., CHOUVET D., MODARESSI H., MOUROUX P. (1985), Local amplification of a seismic incident field through an elastoplastic sedimentary valley. Numerical Methods in Geomechanics, (Ed. Kawamoto, Ichikawa), Balkema, 421-428. 
AUBRY D., CHOUVET D., MODARESSI A., MODARESSI H. (1985), GEYDYN : Logiciel d'analyse du comportement mécanique des sols par éléments finis avec prise en compte du couplage sol-eau-air. Rapport EdF/Région d'équipement Chambéry.

AURIAULT J.L. (1984), Relations entre les comporte. ments Micros et Macros structuraux. Ecole d'hiver de Rhéologie des Géomatériaux. Aussois France.

BARD P.Y, BOUCHON M. (1980), The seismic response of sediment-filled valleys. Part 1 : The case of incident SH waves. B.S.S.A., vol, $70, n^{\circ} 4$, 1263-1286.

BARD P.Y, BOUCHON M. (1980), The seismic response of sediment.filled valleys. Part 2: The case of incident $P$ and SV waves. B.S.S.A., vol. 70 , $n^{\circ} 5,1921-1941$.

BARD P.Y. (1982), Diffracted waves and displacement field over two-dimensional elevated topographies. Geophys. J.R. Soc., 731-760.

BARD P.Y. (1983), Les effets de site d'origine structurale en sismologie; Modélisation et interprétation. Application au risque sismique. Thèse d'état. Université de Grenoble.

BARD P.Y. (1985), Les effets de site d'origine structurale: Principaux résultats expérimentaux et théoriques. Génie para-sismique (V. Davidovici ed.), Presses ENPC, 224-238.

BEAR J. (1984), Fundamentals of Transport Phenomena in Porous Media. Martinus Nijhoff Publishers.

BELYTSCHKO T., SCHOEBERLE D.F. (1975), On the unconditional stability of an implicit algorithm for nonlinear structural dynamics. Jou. of Appl. Mech., vol. $42, n^{\circ} 4,865-869$.

BIOT M.A. (1941), General theory of three-dimensional consolidation. Journal of Applied Physics, vol. 12. 155-164.

BIOT M.A. (1956), Theory of deformation of a porous viscoelastic anisotropic solid. Journal of Applied Physics, vol, 27, n 5, 459-467.

BIOT M.A. (1962), Mechanics of deformation and acoustic propagation in porous media. Journal of Applied Physics, vol. 33, n4, 1482-1498.

BIOT M.A. (1962), Theory of propagation of elastic waves in a fluid-saturated porous solid I-Low frequency range. The journal of Acoustical Society of America, vol. 28, $\mathrm{n}^{\circ} 2,168-178$.

BIOT M.A. (1962), Theory of propagation of elastic waves in fluid-saturated porous solid II-Higher frequency range. The Journal of Acoustical Society of America, vol. 28, $n^{\circ} 2,179-191$.

BOELLE J.L. (1983), Mesure en régime dynamique des propriétés mécaniques des sols aux faibles déformations. Thèse DDI, Ecole Centrale des Arts et Manufactures.

BOORE D.M., HARMSEN S.C., HARDING S.T. (1981). Wave scattering from a step change in surface topography. B.S.S.A., vol. 71, $\mathrm{N}^{\circ} 1,117-125$.

BOORE D.M., JOYNER W.B. (1982), The emprical prediction of ground motion. B.S.S.A, vol. 72, $\mathrm{n}^{\circ} 6, \mathrm{~S} 43-\mathrm{S} 60$.
BORNE L. (1983). Contribution à l'étude du compor. tement dynamique des milieux poreux saturés déformables. Etude de la loi de filtration dynamique. Thèse DDI, Université de Grenoble.

BOUCHON M. (1973), Effect of topography on surface motion. B.S.S.A., vol. $63, \mathrm{n}^{\circ} 3,615-632$.

BOWEN R.M. (1976), Theory of mixtures. Continuum Physics. (Ed. A.C. Eringen), vol. 3, Academic Press.

CLAYTON R., ENGQUIST B. (1977), Absorbing boundary conditions for acoustic and elastic wave equations. B.S.S.A., vol. 67, $n^{\circ} 6,1529-1540$.

CHOUVET D. (1983), Calcul élastoplastique d'interaction dynamique sols-structure: Application aux ouurages de soutènement. Thèse DDI. Ecole Centrale des Arts et Manufactures.

COHEN M., JENNINGS P.C. (1983), Silent boundary methods for transient analysis. Computational methods for transient analysis. (Ed. Belytschko, Hugues) Elsevier.

DRAVINSKI M. (1982), Influence of interface depth upon strong ground motion. B.S.S.A., vol. 72 $n^{\circ} 2,597-614$.

DRAVINSKI M. (1983), Amplification of P SV and Ray. leigh waves by two alluvial valleys. Soil Dynamics \& Earthquake Engineering, vol. 2, 66-77.

ELGAMAL A.W.M., ABDEL-GHAFFAR A.M., PREVOST J.H. (1985), Elasto-plastic earthquake-shear response of one-dimensional earth dam models. Earthquake Eng. \& Struc. Dyn., vol. 13, 617-633.

EL HOSRI M.S. (1984), Contribution à l'étude des propriétés mécaniques des matériaux. Thèse d'état Université Paris VI.

ENGQUIST B., MAJDA A. (1977), Absorbing boundary conditions for the numerical simulation of waves. Mathematics of Computation, vol. 31, $\mathrm{n}^{\circ} 139$, 629-651.

ENGQUIST B., MAJDA A. (1979), Radiation boundary conditions for acoustic and elastic wave calculations. Communications on pure and applied Mathematics, vol. XXXII, $n^{\circ} 3,313-357$.

GELI L. (1985), Propagation d'ondes sismiques dans les formations superficielles : effet d'un arrangement géométrique complexe et influence de la saturation en eau. Thèse de Doctorat. Université de Grenoble.

HAJAL T. (1984), Modélisation élastoplastique des sols par une loi multimécanisme: Application au calcul pressiométrique. Thèse DDI. Ecole Centrale des Arts et Manufactures.

HARMSEN S., HARDING S. (1981), Surface motion over a sedimentary valley for incident plane $P$ and SV waves. B.S.S.A., vol. $71, n^{\circ} 3,665-670$.

HICHER P.Y. (1985), Comportement des argiles saturées sur divers chemins de sollicitations monotones et cycliques. Application à une modélisation élasto. plastique et viscoplastique. Thèse d'état -Université Paris VI.

HUGHES T.J.R., LIU W.K. (1978), Implicit - explicit finite elements in transient analysis : Stability theory. Journal of Applied Mechanics, vol. 45, 371-375. 
HUGHES T.J.R., LIU W.K. (1978), Implicit -explicit finite elements in transient analysis: Implementation and numerical examples. Journal of Applied Mechanics, vol. $45,375-378$.

HUJEUX J.C. (1985), Une loi de comportement pour le chargement cyclique des sols. Génie parasismique (V. Davidovici ed.). Presses ENPC, 287-302.

IDRISS I.M., SEED H.B. (1968), Seismic response of horizontal soil layers. J. Soil Mech. Found. Div. ASCE, vol. 94.

ISHIHARA K. (1982), Evaluation of soil properties for use in earthquake response analysis. Int. Symp. on Num. Mod. in Goemech., Zurich, 237-259.

JOHNSON L.R., SILVA W. (1981), The effects of unconsolidated sediments upon the ground motion local earthquakes, B.S.S.A., vol. $71, n^{\circ} 1$, $127-142$.

JOYNER W.B., CHEN A.T.F. (1975), Calculation of nonlinear ground response in earthquakes. B.S.S.A., vol. $65, n^{\circ} 5,1315-1336$.

JOYNER W.B. (1975), A method for calculating nonlinear seismic response in two dimensions. B.S.S.A., vol. $65, n^{\circ} 5,1337-1357$.

JOYNER W.B., WARRICK R.E., FUMAL T.E. (1981). The effects of quaternary alluvium on strong ground motion in the Coyote lake. California earthquake of 1979. B.S.S.A., vol. 71, $\mathrm{n}^{\circ} 4,1333-1349$.

KING J.L., BRUNE J.N. (1981), Modeling the seismic response of sedimentary basins. B.S.S.A., vol. 71, $n^{\circ} 5,1469-1487$.

KUHLEMEYER R.L., LYSMER J. (1973), Finite element method accuracy for wave propagation pro. blems. Jou. of Soil Mech. and Found. Div. ASCE, 421-426.

LUCO J.E. (1980), Linear soil-structure interaction. Report UCRL-15272. Lawrence Livermore National Laboratory.

MOHAMMADIOUM B., PECKER A. (1984), Lowfrequency transfer of seismic energy by superfacial soil deposits and soft rocks. Earthquake Eng. \& Struc. Dyn., vol. 12, 537-564.

MODARESSI H. (1987), Modélisation numérique de la propagation des ondes dans les milieux poreux anélastiques. Thèse de Doctorat. Ecole Centrale de Paris.

OHTSUKI A., HARUMI K. (1983), Effects of topography and subsurface inhomogeneities on seismic SV waves. Earthquake Eng. \& Struc. Dyn., vol. 11, 441-462.

OHTSUKI A., YAMAHARA H., HARUMI K. (1984), Effects of topography and subsurface inhomogeneities on seismic Rayleigh waves. Earthquake Eng. \& Struc. Dyn., vol. 11, 37-58.

OHTSUKI A., YAMAHARA H., TAZOH T. (1984), Effects of lateral inhomogeneities on seismic waves. II: Observation and analysis. Earthquake Eng. \& Struc. Dyn., vol. 12, 795-816.

PECKER A. (1985), Comportement des sols sous chargement cyclique. Génie para-sismique (V. Davidovici ed.), Presses ENPC, 275-285.

PECKER A. (1985), Réponse d'un profil de sol en champ libre. Génie para-sismique (V. Davidovici ed.). Presses ENPC, 313-322.

RICKER N. (1960), The form and laws of propagation of seismic wavelets. Geophysics, vol. 18, 10,40.

TOKI K., SATO T. (1977), Seismic response analysis of surface layer with irregular boundaries. Proc. 6th World Conf. on Earthquake Eng., vol. 2, 81-86. Newdelhi, India.

ZIENKIEWICZ O.C., SHIOMI T. (1984), Dynamic beha. viour of saturated porous media: The generalized Biot formulation and its numerical solution. Int. JOU. Num. and Anal. Meth. Geomech., vol. 8, 71-96.

ZIENKIEWICZ O.C. (1985), The coupled problems of soil-pore fluid-external flluid interaction basis for a general geomechanics code. Fifth International Conference on Numerical Methods in Geomechanics. Nagoya, 1731-1740. 